\title{
Theory of Non-Equilibrium Stationary States as a Theory of Resonances
}

\author{
Marco Merkli*, Matthias Mück ${ }^{\dagger}$, and Israel Michael Sigal ${ }^{\ddagger}$
}

\begin{abstract}
We study a small quantum system (e.g., a simplified model for an atom or molecule) interacting with two bosonic or fermionic reservoirs (say, photon or phonon fields). We show that the combined system has a family of stationary states parametrized by two numbers, $T_{1}$ and $T_{2}$ ('reservoir temperatures'). If $T_{1} \neq T_{2}$, then these states are non-equilibrium stationary states (NESS). In the latter case we show that they have nonvanishing heat fluxes and positive entropy production and are dynamically asymptotically stable. The latter means that the evolution with an initial condition, normal with respect to any state where the reservoirs are in equilibria at temperatures $T_{1}$ and $T_{2}$, converges to the corresponding NESS. Our results are valid for the temperatures satisfying the bound $\min \left(T_{1}, T_{2}\right)>g^{2+\alpha}$, where $g$ is the coupling constant and $0<\alpha<1$ is a power related to the infra-red behaviour of the coupling functions.
\end{abstract}

\section{Introduction}

The present paper is a contribution to rigorous quantum statistical mechanics. Key problems here are dynamical stability of equilibrium states, and characterization (if not the definition) and stability of non-equilibrium stationary states (NESS).

While our understanding of the quantum equilibrium states, the subject of equilibrium statistical mechanics (see $[9,16,17,31]$ ), and the recent progress in proving their dynamical stability $[8,10,11,14,18,22,23]$ are satisfactory, results on non-equilibrium stationary states are just beginning to emerge. The problem is that we do not have a simple stationary characterization of NESS similar to

* Partly supported by an NSERC PDF, the Institute of Theoretical Physics of ETH Zürich, Switzerland, the Departments of Mathematics of McGill University and the University of Toronto, Canada.

† Supported by DAAD under grant HSP III.

¥ Supported by NSERC under grant NA7901. 
the principle of maximum of entropy or the KMS characterization for equilibrium states. Thus it is remarkable that certain characterizations of NESS and their stability were recently shown for (idealized) particle systems coupled to Fermi reservoirs at high temperatures, $\min \left(T_{1}, T_{2}\right)>C\left[\ln \frac{1}{g}\right]^{-1}$, in [21], for XY-chains [4] and for coupled Fermi reservoirs in $[1,2,12,15]$. Here, $T_{1,2}$ are the temperatures of the reservoirs, and $g$ is the coupling constant, which is assumed to be sufficiently small.

There are two rigorous approaches to non-equilibrium, quantum statistical mechanics. One is based on scattering theory - wave (or Møller) morphisms - and the other, on the theory of resonances via complex deformations. In this paper we follow the second approach which we believe applies to a wider class of physical models.

In this paper we establish a spectral characterization of the NESS and prove their dynamical stability for (idealized) particle systems coupled to two Bose reservoirs (e.g., photons or phonons) for reservoir temperatures satisfying $\min \left(T_{1}, T_{2}\right)>$ $C g^{2+\alpha}$, where $C$ is a constant and $0<\alpha<1\left(\alpha=\frac{\mu-1 / 2}{\mu+1 / 2}\right.$, where $\mu>1 / 2$ is given in Condition (B) below). Our approach applies to an arbitrary finite number of bosonic or fermionic reservoirs; in the latter case, it gives an extension of the results of [21] to the temperature range mentioned above. Moreover, we develop a perturbation theory for NESS and use it to prove that the entropy production is strictly positive.

An appropriate iteration of our estimates in the spirit of the spectral renormalization group of [5-8] would give the above results for all temperatures. This extension will be presented elsewhere.

Similarly to [21], we construct a NESS from a zero (non-degenerate) resonance eigenvector of a certain non-self-adjoint Liouville operator, $K$, acting on a positive temperature Hilbert space. The operator $K$ is the analogue of a $C$-Liouvillean in the terminology for $C^{*}$-dynamical systems [21].

To show dynamical stability of a NESS we have to establish certain long-time (ergodic) properties of the evolution, $U(t)$, generated by $K$. The operator $K$ does not belong to a class for which the evolution is a priori known to exist (e.g., a class of normal or accretive operators). To overcome this problem we establish a direct connection between desired ergodic properties of $U(t)$ and certain spectral properties of a complex deformation, $K_{\theta}, \theta \in \mathbb{C}^{2}$, of $K$. For technical reasons we can use neither the complex deformations introduced in [18] nor those introduced in [8] but we combine both types, hence $\theta$ is in $\mathbb{C}^{2}$ rather than in $\mathbb{C}$. (Such a combination was already mentioned in [8]). In order to establish the desired spectral characteristics of the operator family $K_{\theta}$, we use the method of the Feshbach map, as developed in [5-7].

The present paper suffers from the main weaknesses shared by all the works in the area, except, in some aspects, of [8]:

(i) The particle system has a finite-dimensional state space;

(ii) The restriction on the coupling functions is severe;

(iii) Temperatures considered are high. 
To overcome the first limitation one would have to go beyond, or at least significantly extend, the present approach. The second limitation is due to use of translation analyticity (which in our case is combined with the dilation analyticity), see Remark 3 in Section 3. This analyticity is used in the present work in a single place - in controlling the nonsingular part of the resolvent of the operator $K_{\theta}$ near the zero resonance pole by rendering this pole isolated and therefore the nonsingular part of the resolvent analytic (see the estimate (8.8)). Without it the zero resonance of the operator $K_{\theta}$ is not separated from the continuous spectrum and sits exactly at a threshold of the latter. Hence to control the nonsingular part of the resolvent near the zero threshold becomes a delicate matter.

The paper [8] has rather mild restrictions on the coupling functions due to using the dilation analyticity. Since [8] deals with the dynamics near equilibrium, the operator $K$ in this case is self-adjoint and an analogue of (8.8) is obtained with help of an abstract spectral theory of self-adjoint operators. Furthermore, [8] handles arbitrary temperatures by employing the spectral renormalization group. In the present paper we take the first step in removing the high temperature restriction. To this end we use the Feshbach map of [7]. Already a single application of the Feshbach map considered in this paper improves the temperature bounds yielding the results mentioned above. We also set the stage for the iteration of this map - the spectral renormalization group method - which would remove the restriction on the temperature altogether. The iteration procedure will be carried out elsewhere. (Note that the works $[12,15]$ deal with arbitrary temperatures, but the scattering approach they use seems to be inapplicable to the models considered in this paper.)

A more detailed outline of our approach and of the organization of the paper is given in Section 2.

\section{Model and approach}

We consider a system consisting of a particle system, described by a self-adjoint Hamiltonian $H_{p}$ on a Hilbert space $\mathcal{H}_{p}$, and two bosonic reservoirs, at inverse temperatures $\beta_{1}$ and $\beta_{2}$, described by the Hamiltonians $H_{r 1}$ and $H_{r 2}$ acting on Hilbert spaces $\mathcal{H}_{r 1}$ and $\mathcal{H}_{r 2}$, respectively. The full Hamiltonian is

$$
H:=H_{0}+g v,
$$

acting on the tensor product space $\mathcal{H}_{0}:=\mathcal{H}_{p} \otimes \mathcal{H}_{r 1} \otimes \mathcal{H}_{r 2}$. Here

$$
H_{0}:=H_{p} \otimes \mathbf{1} \otimes \mathbf{1}+\mathbf{1} \otimes H_{r 1} \otimes \mathbf{1}+\mathbf{1} \otimes \mathbf{1} \otimes H_{r 2}
$$

is the unperturbed Hamiltonian, $v$ is an operator on $\mathcal{H}_{0}$ describing the interaction and $g \in \mathbb{R}$ is a coupling constant.

For our key results we have to assume that the space $\mathcal{H}_{p}$ is finite-dimensional, through some of the results hold for infinite-dimensional spaces. 
The operators $H_{r j}$ describe free scalar (or vector, if wished) quantum fields on $\mathcal{H}_{r j}$, the bosonic Fock spaces over the one-particle space $L^{2}\left(\mathbb{R}^{3}, d^{3} k\right)$,

$$
H_{r j}=\int \omega(k) a_{j}^{*}(k) a_{j}(k) d^{3} k,
$$

where $a_{j}^{*}(k)$ and $a_{j}(k)$ are creation and annihilation operators on $\mathcal{H}_{r j}$ and $\omega(k)=$ $|k|$ is the dispersion relation for relativistic massless bosons. The interaction operator is given by

$$
v=\sum_{j=1}^{2} v_{j} \quad \text { with } \quad v_{j}=a_{j}\left(G_{j}\right)+a_{j}^{*}\left(G_{j}\right) .
$$

Its choice is motivated by standard models of particles interacting with the quantized electromagnetic field or with phonons.

In (2.4), $G_{j}: k \mapsto G_{j}(k)$ is a map from $\mathbb{R}^{3}$ into $\mathcal{B}\left(\mathcal{H}_{p}\right)$, the algebra of bounded operators on $\mathcal{H}_{p}$, and

$$
a_{j}\left(G_{j}\right):=\int G_{j}(k)^{*} \otimes a_{j}(k) d^{3} k \quad \text { and } \quad a_{j}^{*}\left(G_{j}\right):=a_{j}\left(G_{j}\right)^{*} .
$$

If the coupling operators $G_{j}$ are such that

$$
g^{2} \int_{\mathbb{R}^{3}}\left(1+|k|^{-1}\right)\left\|G_{j}(k)\right\|^{2} d k \quad \text { is sufficiently small, }
$$

then the operator $H$ is self-adjoint (see e.g., [8]).

Now we set up a mathematical framework for non-equilibrium statistical mechanics. Operators on the Hilbert space $\mathcal{H}_{0}$ will be called observables. (Only certain self-adjoint operators on $\mathcal{H}_{0}$ are actually physical observables.) As an algebra of observables describing the system we take the $C^{*}$-algebra

$$
\mathcal{A}=\mathcal{B}\left(\mathcal{H}_{p}\right) \otimes \mathfrak{W}\left(L_{0}^{2}\right) \otimes \mathfrak{W}\left(L_{0}^{2}\right),
$$

where $\mathfrak{W}\left(L_{0}^{2}\right)$ denotes the Weyl CCR algebra over $L_{0}^{2}:=L^{2}\left(\mathbb{R}^{3},\left(1+|k|^{-1}\right) d^{3} k\right)$, i.e., the $C^{*}$-algebra generated by the Weyl operators $W_{j}(f):=e^{i \phi_{j}(f)}, \phi_{j}(f):=$ $\frac{1}{\sqrt{2}}\left(a_{j}^{*}(f)+a_{j}(f)\right)$, with $f \in L_{0}^{2}$, see, e.g., [9]. States of the system are positive linear ('expectation') functionals $\psi$ on the algebra $\mathcal{A}$, normalized as $\psi(\mathbf{1})=1$.

The reason we chose $\mathcal{A}$ rather than $\mathcal{B}\left(\mathcal{H}_{0}\right)$ is that the algebra $\mathcal{A}$ supports states in which each reservoir is at a thermal equilibrium at its own temperature. More precisely, consider the evolution for the $j$-th reservoir given by

$$
\alpha_{r j}^{t}(A):=e^{i H_{r j} t} A e^{-i H_{r j} t} .
$$

Then there are stationary states on the $j$-th reservoir algebra of observables, $\mathfrak{W}\left(L_{0}^{2}\right)$, which describe (single-phase) thermal equilibria. These states are parametrized by the inverse temperature $\beta_{j}=1 / T_{j}$ and their generating functional is given by

$$
\omega_{r j}^{\left(\beta_{j}\right)}\left(W_{j}(f)\right)=\exp \left\{-\frac{1}{4} \int_{\mathbb{R}^{3}} \frac{e^{\beta_{j}|k|}+1}{e^{\beta_{j}|k|}-1}|f(k)|^{2} d^{3} k\right\} .
$$


The choice of the space $L_{0}^{2}$ above is dictated by the need to have the r.h.s. of this functional finite. These states are characterized by the KMS condition and are called the $\left(\alpha_{r j}^{t}, \beta_{j}\right)$-KMS states.

Remark. It is convenient to define states $\psi$ on products $a^{\#}\left(f_{1}\right) \ldots a^{\#}\left(f_{n}\right)$ of the creation and annihilation operators, where $a^{\#}$ denotes either $a$ or $a^{*}$. This is done using derivatives $\partial_{s_{k}}$ of its values on the Weyl operators $W\left(s_{1} f_{1}\right) \ldots W\left(s_{n} f_{n}\right)$ (see [9], Section 5.2.3 and (2.15)).

Consider states $($ on $\mathcal{A}$ ) of the form

$$
\omega_{0}:=\omega_{p} \otimes \omega_{r 1}^{\left(\beta_{1}\right)} \otimes \omega_{r 2}^{\left(\beta_{2}\right)},
$$

where $\omega_{p}$ is a state of the particle system and $\omega_{r i}^{(\beta)}$ is the $\left(\alpha_{r i}^{t}, \beta\right)$-KMS state of the $i$-th reservoir. The set of states which are normal w.r.t. $\omega_{0}$ is the same for any choice of $\omega_{p}$. A state normal w.r.t. $\omega_{0}$ will be called a $\beta_{1} \beta_{2}$-normal state.

In the particular case $\omega_{p}(\cdot)=\operatorname{Tr}\left(e^{-\beta_{p} H_{p}} \cdot\right) / \operatorname{Tr}\left(e^{-\beta_{p} H_{p}}\right)$ we call $\omega_{0}$ a reference state.

The Hamiltonian $H$ generates the dynamics of observables $A \in \mathcal{B}\left(\mathcal{H}_{0}\right)$ according to the rule

$$
A \mapsto \alpha^{t}(A):=e^{i H t} A e^{-i H t} .
$$

Equation (2.11) defines a group of ${ }^{*}$-automorphisms of $\mathcal{B}\left(\mathcal{H}_{0}\right)$. However, $\alpha^{t}$ does not map the subalgebra $\mathcal{A} \subset \mathcal{B}\left(\mathcal{H}_{0}\right)$ into itself, so (2.11) does not define a dynamics on $\mathcal{A}$. To circumvent this problem we define the interacting evolution of a class of states on $\mathcal{A}$ by using the Araki-Dyson expansion. Namely, we define the evolution of a state $\psi$ on $\mathcal{A}$ which is normal w.r.t. $\omega_{0}$ by

$$
\psi^{t}(A):=\lim _{n \rightarrow \infty} \sum_{m=0}^{\infty}(i g)^{m} \int_{0}^{t} d t_{1} \cdots \int_{0}^{t_{m-1}} d t_{m} \psi_{n}^{t, t_{1}, \ldots, t_{m}}(A),
$$

where the term with $m=0$ is $\psi\left(\alpha_{0}^{t}(A)\right)$, and, for $m \geq 1$,

$$
\psi_{n}^{t, t_{1}, \ldots, t_{m}}(A):=\psi\left(\left[\alpha_{0}^{t_{m}}\left(v_{n}\right), \cdots\left[\alpha_{0}^{t_{1}}\left(v_{n}\right), \alpha_{0}^{t}(A)\right] \cdots\right]\right) .
$$

Here, $v_{n}=v_{n}^{*} \in \mathcal{A}$ is an approximating sequence for the operator $v$, satisfying the relation

$$
\lim _{n \rightarrow \infty} \omega_{0}\left(A^{*}\left(v_{n}^{*}-v^{*}\right)\left(v_{n}-v\right) A\right)=0,
$$

for all $A$ polynomials in $a_{j}^{*}(f), j=1,2, f \in L_{0}^{2}$. Such a sequence is constructed as follows. Let $\left\{e_{m}\right\}$ be an orthonormal basis in $L_{0}^{2}$. We define the approximate creation operators

$$
a_{j, n}^{*}\left(G_{j}\right)=\sum_{m=1}^{\mu}\left\langle e_{m}, G_{j}\right\rangle b_{j, \lambda}^{*}\left(e_{m}\right),
$$

where $n=(\lambda, \mu)$, and, for any $f \in L^{2}\left(\mathbb{R}^{3}\right)$ and $\lambda>0$,

$$
b_{j, \lambda}^{*}(f):=\frac{\lambda}{\sqrt{2} i}\left\{W_{j}(f / \lambda)-\mathbf{1}-i W_{j}(i f / \lambda)+i \mathbf{1}\right\} .
$$


Similarly we define the approximate annihilation operators $a_{j, n}\left(G_{j}\right)$. These operators belong to $\mathcal{A}$. Via the above construction we obtain the family of interactions $v_{n}$ which belongs to $\mathcal{A}$ and, as can be easily shown using (2.9), satisfies (2.13).

We show in Appendix A that under condition (2.13) the integrands on the r.h.s. of (2.12) are continuous functions in $t_{1}, \ldots, t_{m}$, that the series is absolutely convergent and that the limit exists and is independent of the approximating sequence $v_{n}$.

Our goal is to understand stationary states of the interacting system originating from $\beta_{1} \beta_{2}$-normal states either by a perturbation theory or through an ergodic limit of the full evolution $\alpha^{t}$. These states are not equilibrium (KMS) states states. They will be called non-equilibrium stationary states or NESS for short. Their main feature is that the energy (heat) fluxes between the reservoirs and the particle system do not vanish.

Assuming certain smoothness and smallness conditions on the coupling operators $g G_{j}(k)$ and assuming that the particle system is effectively coupled to the reservoirs, we show that, starting initially in any $\beta_{1} \beta_{2}$-normal state $\psi$, the system converges, under the evolution $\alpha^{t}$, to a state $\eta$ :

$$
\psi^{t} \longrightarrow \eta \quad \text { as } \quad t \rightarrow \infty \text {. }
$$

The convergence $(2.16)$ is understood in the weak* sense on the sub- $C^{*}$-algebra of "analytic observables"

$$
\mathcal{A}_{1}=\mathcal{B}\left(\mathcal{H}_{p}\right) \otimes \mathfrak{W}\left(D^{\text {anal }}\right) \otimes \mathfrak{W}\left(D^{\text {anal }}\right) .
$$

Here, $\mathfrak{W}\left(D^{\text {anal }}\right)$ is the Weyl CCR algebra over the dense set $D^{\text {anal }} \subset L_{0}^{2}$ which we define in Appendix C. Roughly speaking, $D^{\text {anal }}$ consists of vectors from the space $\bigcap_{b \geq 0} e^{-b|k|} L_{0}^{2}$ which have some analyticity properties in $|k|$ and a certain behaviour at $k=0$. The density of $D^{\text {anal }} \subset L_{0}^{2}$ implies that $\mathcal{A}_{1}$ is strongly dense in $\mathcal{A}$. The construction of the state $\eta$ and the proof of its stability, (2.16), will rely on the theory of resonances for the evolution $\psi \rightarrow \psi^{t}$.

As mentioned in the introduction, so far, we do not have a simple characterization of NESS. However, there is a key physical quantity which differentiates between equilibrium and non-equilibrium stationary states - the collection of heat fluxes. In our case, the heat flux, or more precisely the heat flow rate (i.e., the energy flow rate due to thermal contact), $\phi_{j}, j=1,2$, into the $j$-th reservoir is given by

$$
\phi_{j}:=\left.\frac{\partial}{\partial t}\right|_{t=0} \alpha^{t}\left(H_{r j}\right),
$$

and the heat flux, $\phi_{0}$, into the particle system is defined as

$$
\phi_{0}:=\left.\frac{\partial}{\partial t}\right|_{t=0} \alpha^{t}\left(H_{p}\right) .
$$

The heat fluxes can be combined into a single quantity - the entropy production. Motivated by the second law of thermodynamics $\left(d S=\sum \beta_{j} d Q_{j}\right)$ we 
introduce the observable of entropy production (rate) as

$$
s:=\sum_{j=0}^{2} \beta_{j} \phi_{j},
$$

where, for notational convenience, we write $\beta_{0}:=\beta_{p}$. The entropy production, $E P(\omega)$, in a state $\omega$ is defined as (see $[19-21,27,28,32-36]$ )

$$
E P(\omega)=\omega(s) .
$$

Since $s$ is not a bounded operator, we have to use an approximation procedure similar to the one mentioned in the remark after (2.9) in order to define the r.h.s. of (2.21) for sufficiently regular states.

The entropy production $E P(\eta)$ of the NESS is independent of the particle state $\omega_{p}$ entering Definition (2.10) of the state of the decoupled system, since $\eta$ is independent of $\omega_{p}$. Notice that $\eta\left(\phi_{0}\right)=\left.\partial_{t}\right|_{t=0} \eta\left(\alpha^{t}\left(H_{p}\right)\right)$, since, by assumption, $H_{p} \in \mathcal{B}\left(\mathcal{H}_{p}\right)$ is a proper observable and $\eta$ is a continuous and stationary state. Hence

$$
\eta\left(\phi_{0}\right)=0 .
$$

Therefore, writing $\sum_{j=1}^{2} \eta\left(\phi_{j}\right)=\sum_{j=0}^{2} \eta\left(\phi_{j}\right)=\eta\left(\left.\partial_{t}\right|_{t=0} \alpha^{t}\left(H_{0}\right)\right)=-\left.\partial_{t}\right|_{t=0} \eta\left(\alpha^{t}(v)\right)$ $=0$, we obtain

$$
\sum_{j=1}^{2} \eta\left(\phi_{j}\right)=0
$$

Observe that the zero total flow relation (2.23) and Definition (2.20) for the entropy production rate imply that

$$
E P(\eta)=\left(\beta_{1}-\beta_{2}\right) \eta\left(\phi_{1}\right) .
$$

Thus, the relation $E P(\eta)>0$ is equivalent to

$$
\eta\left(\phi_{1}\right)>0 \text { whenever } T_{2}>T_{1},
$$

where $T_{j}=\beta_{j}^{-1}$ is the temperature of the $j$-th reservoir. In other words, in the state $\eta$ the energy flows from the hotter to the colder reservoir.

A general result due to [19] shows that $E P(\omega) \geq 0$ for any NESS $\omega$. We show that for the NESS $\eta$,

$$
E P(\eta)>0 \quad \text { iff } \quad \beta_{1} \neq \beta_{2},
$$

see Theorem 3.2 and Section 12 for a precise statement of this result. Moreover, we develop a perturbation theory for the NESS and compute $E P(\eta)$ in leading order in the coupling constant $g$.

Let us outline the main steps of our proof of the convergence (2.16) (c.f. [21]). We pass to the Araki-Woods GNS representation of $\left(\mathcal{A}, \omega_{0}\right)$, with $\omega_{0}$ of the form $(2.10)$ and $\omega_{p}(A):=\operatorname{Tr}\left(e^{-\beta_{p} H_{p}} A\right) / \operatorname{Tr}\left(e^{-\beta_{p} H_{p}}\right)$;

$$
\left(\mathcal{A}, \omega_{0}\right) \rightarrow\left(\mathcal{H}, \pi, \Omega_{0}\right),
$$


where $\mathcal{H}, \pi$ and $\Omega_{0}$ are a Hilbert space, a representation of the algebra $\mathcal{A}$ by bounded operators on $\mathcal{H}$, and a cyclic element in $\mathcal{H}$ (meaning that $\overline{\pi(\mathcal{A}) \Omega_{0}}=\mathcal{H}$ ) s.t.

$$
\omega_{0}(A)=\left\langle\Omega_{0}, \pi(A) \Omega_{0}\right\rangle .
$$

The GNS representation provides us with a Hilbert space framework which we use to convert the dynamical problem described above into a spectral problem for a certain non-self-adjoint operator $K$ on the Hilbert space $\mathcal{H}$. With the free evolution $\alpha_{0}^{t}(A):=e^{i t H_{0}} A e^{-i t H_{0}}$ one associates the unitary one-parameter group $U_{0}(t)=e^{i t L_{0}}$ on $\mathcal{H}$ s.t.

$$
\pi\left(\alpha_{0}^{t}(A)\right)=U_{0}(t) \pi(A) U_{0}(t)^{-1}
$$

and $U_{0}(t) \Omega_{0}=\Omega_{0}$. Define the operator $L^{(\ell)}:=L_{0}+g \pi(v)$ on the dense domain $\operatorname{Dom}\left(L_{0}\right) \cap \operatorname{Dom}(\pi(v))$. Here $\pi(v)$ can be defined either using explicit formulae for $\pi$ in the Araki-Woods representation given below or by using the approximation, $v_{n} \in \mathcal{A}$, for the operator $v$ constructed above. By the Glimm-Jaffe-Nelson commutator theorem the operator $L^{(\ell)}$ is essentially self-adjoint; we denote its self-adjoint closure again by the same symbol $L^{(\ell)}$. The operator $L^{(\ell)}$ induces the one-parameter group $\sigma^{t}$ on $\pi(\mathcal{A})^{\prime \prime}$, the weak closure of $\pi(\mathcal{A})$,

$$
\sigma^{t}(B):=e^{i t L^{(\ell)}} B e^{-i t L^{(\ell)}}
$$

for any $B \in \pi(\mathcal{A})^{\prime \prime}$. Let $\psi$ be a state on the algebra $\mathcal{A}$ normal w.r.t. $\omega_{0}$, i.e.,

$$
\psi(A)=\operatorname{Tr}(\rho \pi(A))
$$

for some positive trace class operator $\rho$ on $\mathcal{H}$ of trace one. It is shown in Appendix A that for $\psi$ as above the limit on the r.h.s. of (2.12) exists and equals

$$
\psi^{t}(A)=\operatorname{Tr}\left(\rho \sigma^{t}(\pi(A))\right) .
$$

In particular, the limit is independent of the choice of the approximating family $v_{n}$.

Due to (2.29) the dynamics on normal states, defined in (2.12), gives rise to the dynamics on the Hilbert space $\mathcal{H}$, determined by a one-parameter group $U(t)$, satisfying

$$
\operatorname{Tr}\left(\rho U(t) \pi(A) U(t)^{-1}\right)=\psi^{t}(A), \quad \forall A \in \mathcal{A} .
$$

Due to the fact that the von Neumann algebra $\pi(\mathcal{A})^{\prime \prime}$ has a large commutant (which isomorphic to $\pi(\mathcal{A})^{\prime \prime}$, as is known from Tomita-Takesaki theory), relation (2.30) does not define $U(t)$ uniquely; however, if we impose in addition to (2.30) the invariance condition

$$
U(t) \Omega=\Omega,
$$

where $\Omega$ is a fixed cyclic and separating vector, then $U(t)$ is uniquely determined. (The vector $\Omega$ is called cyclic if $\pi(\mathcal{A}) \Omega$ is dense in $\mathcal{H}$ and separating if $\pi(\mathcal{A})^{\prime} \Omega$ is dense in $\mathcal{H}$, the prime denoting the commutant.) If $\Omega$ were the vector representing an equilibrium state then $U(t)$ satisfying (2.30) and (2.31) would be a unitary group. In the non-equilibrium case $\beta_{1} \neq \beta_{2}$, one can see that (2.31) cannot be satisfied for a unitary $U(t)$ implementing the dynamics as in (2.30). For technical 
reasons, we choose $U(t)$ to satisfy (2.31) for a convenient vector $\Omega$, rather than to be unitary (cf. [21]).

We will show that $U(t)$ is strongly differentiable on a dense set of vectors and we will calculate explicitly its generator, $K:=-\left.i \frac{\partial}{\partial t} U(t)\right|_{t=0}$. In the nonequilibrium situation $K^{*} \neq K(U(t)$ is not unitary!) and (2.31) implies that $K \Omega=0$. The main effort of our analysis is to derive enough spectral information on the operator $K$ to enable us to show (2.16) and to identify the NESS with

$$
\eta(A)=\left\langle\Omega^{*}, \pi(A) \Omega\right\rangle,
$$

where $\Omega^{*}$ is a zero resonance of the operator $K^{*}: K^{*} \Omega^{*}=0$ (in the sense of distributions) and $\Omega^{*} \in \mathcal{D}_{\text {anal }}^{\prime}$, for an appropriate dense set $\mathcal{D}_{\text {anal }} \subset \mathcal{H}$, and $A$ are such that $\pi(A) \Omega \in \mathcal{D}_{\text {anal }}$.

In order to obtain rather subtle spectral information on the operator $K$, and to give a precise meaning to expression (2.32), we develop a new type of spectral deformation, $K \mapsto K_{\theta}$, with a spectral deformation parameter $\theta \in \mathbb{C}^{2}$, in combination with an application of a Feshbach map acting on $K_{\theta}$.

In conclusion of this outline we present here the GNS triple provided by the Araki-Woods construction, which forms a mathematical framework for our analysis (see $[8,9,18]$ for details and $[3,17]$ for original papers). In the ArakiWoods GNS representation the (positive temperature) Hilbert space is given by

$$
\mathcal{H}=\mathcal{H}^{p} \otimes \mathcal{H}^{r},
$$

where $\mathcal{H}^{p}=\mathcal{H}_{p} \otimes \mathcal{H}_{p}$ and $\mathcal{H}^{r}=\mathcal{H}^{r 1} \otimes \mathcal{H}^{r 2}$ with

$$
\mathcal{H}^{r j}=\mathcal{H}_{r j} \otimes \mathcal{H}_{r j}
$$

We denote by $a_{\ell, j}^{\#}(f)$ (resp., $\left.a_{r, j}^{\#}(f)\right)$ the creation and annihilation operators which act on the left (resp., right) factor of (2.34). They are related to the zero temperature creation and annihilation operators $a_{j}^{\#}(f)$ by

$$
\pi\left(a_{j}(f)\right)=a_{\ell j}\left(\sqrt{1+\rho_{j}} f\right)+a_{r j}^{*}\left(\sqrt{\rho_{j}} \bar{f}\right)
$$

and

$$
\pi^{\prime}\left(a_{j}(f)\right)=a_{\ell j}^{*}\left(\sqrt{\rho_{j}} f\right)+a_{r j}\left(\sqrt{1+\rho_{j}} \bar{f}\right)
$$

where $\rho_{j} \equiv \rho_{j}(k)=\left(e^{\beta_{j} \omega(k)}-1\right)^{-1}$ with $\omega(k)=|k|$. Finally, we denote $\Omega_{r}:=$ $\Omega_{r 1} \otimes \Omega_{r 2}$, where $\Omega_{r j}:=\Omega_{r j, \ell} \otimes \Omega_{r j, r}$ are the vacua in $\mathcal{H}^{r j}$. Thus, $\Omega_{r}$ is the vacuum in $\mathcal{H}^{r}$.

Definition (2.10) and our choice of $\omega_{p}$ made at the beginning of this section imply that

$$
\Omega_{0}=\Omega_{p} \otimes \Omega_{r} \quad \text { with } \quad \Omega_{p} \equiv \Omega_{p}^{\left(\beta_{p}\right)}=\frac{\sum_{j} e^{-\beta_{p} E_{j} / 2} \varphi_{j} \otimes \varphi_{j}}{\left[\sum_{j} e^{-\beta_{p} E_{j}}\right]^{1 / 2}},
$$

where $E_{j}$ and $\varphi_{j}$ are the eigenvalues and normalized eigenvectors of $H_{p}$. 
The self-adjoint operator $L_{0}$ generating the free evolution $U_{0}(t)$ defined in (2.26) is of the form $L_{0}=L_{p} \otimes \mathbf{1}^{r}+\mathbf{1}^{p} \otimes L_{r}$ with $L_{r}=\sum_{j=1}^{2} L_{r j}$. The operator $L_{p}$ has the standard form

$$
L_{p}=H_{p} \otimes \mathbf{1}_{p}-\mathbf{1}_{p} \otimes H_{p}
$$

and

$$
L_{r j}=\int \omega(k)\left(a_{\ell, j}^{*}(k) a_{\ell, j}(k)-a_{r, j}^{*}(k) a_{r, j}(k)\right) d^{3} k .
$$

The operator $K$ can be written as $K=L_{0}+g(V-W)$ with $V=\pi(v)$ and $W=\pi^{\prime}(w)$ with $w$ a non-self-adjoint operator obtained by a simple transformation of $v$.

A standard argument shows that the spectrum of the operator $L_{0}$ fills the axis $\mathbb{R}$ with the thresholds and eigenvalues located at $\sigma\left(L_{p}\right)$ and with 0 an eigenvalue of multiplicity at least $\operatorname{dim} H_{p}$ and at most $\left(\operatorname{dim} H_{p}\right)^{2}$ (depending on the degeneracy of the spectrum of $L_{p}$ ). A priori we do not know anything about the spectrum of the non-self-adjoint operator $K$ besides the fact that it has an eigenvalue 0 . For all we know its spectrum might fill in the entire complex plane! Thus understanding the evolution generated by the operator $K$ is a subtle matter.

This paper is organized as follows. In Section 3 we give a precise formulation of our assumptions, state the results and discuss assumptions and results. In Section 4 we present the Hilbert space framework and define the vector $\Omega$ and the evolution $U(t)$ and in Section 5 we describe the generator $K$. In Section 6 we introduce the complex deformation $K_{\theta}$ of $K$ and establish the connection between the resolvents of $K$ and $K_{\theta}$. In Section 7 we establish the spectral properties of $K_{\theta}$ which we then use in Section 8 to express the dynamics in terms of an integral over the resolvent of $K_{\theta}$. In Section 9 we prove our first main result, the existence and explicit form of the NESS, and its dynamical stability. In Section 11 we develop a perturbation theory for NESS and in Section 12 we prove the positivity of the entropy production. Finally, in Appendices A-D we collect some technical results. Section 10 contains a supplementary result on level shift operators.

\section{Assumptions and results}

In order to state assumption (B) below, it is practical to define the map $\gamma$ : $L^{2}\left(\mathbb{R}^{3}\right) \rightarrow L^{2}\left(\mathbb{R} \times S^{2}\right)$,

$$
(\gamma f)(u, \sigma)=\sqrt{|u|} \begin{cases}f(u \sigma), & u \geq 0 \\ -\bar{f}(-u \sigma), & u<0\end{cases}
$$

Let $j_{\theta}(u)=e^{\delta \operatorname{sgn}(u)} u+\tau$ for $\theta=(\delta, \tau) \in \mathbb{C}^{2}$ and $u \in \mathbb{R}$ (see (B.2.2)) and define $\left(\gamma_{\theta} f\right)(u, \sigma)=(\gamma f)\left(j_{\theta}(u), \sigma\right)$, for $f \in L^{2}\left(\mathbb{R} \times S^{2}\right), \theta \in \mathbb{R}^{2}$. The maps $\gamma$ and $\gamma_{\theta}$ have obvious extensions to operator valued functions.

(A) Ultraviolet cut-off. $\int\left\|G_{j}(k)\right\|^{2} e^{a|k|^{2}}\left[1+|k|^{-1}\right] d^{3} k<\infty$ for some $a>0$. 
(B) Analyticity. For $j=1,2$ and every fixed $(u, \sigma) \in \mathbb{R} \times S^{2}$, the maps

$$
\theta \mapsto\left(\gamma_{\theta} G_{j}\right)(u, \sigma)
$$

from $\mathbb{R}^{2}$ to the bounded operators on $\mathcal{H}_{p}$ have analytic continuations to

$$
\left\{(\delta, \tau) \in \mathbb{C}^{2}|| \operatorname{Im} \delta\left|<\delta_{0},\right| \tau \mid<\tau_{0}\right\},
$$

for some $\delta_{0}, \tau_{0}>0, \frac{\tau_{0}}{\cos \delta_{0}} \leq \frac{2 \pi}{\beta}$, where $\beta=\max \left(\beta_{1}, \beta_{2}\right)$. Moreover,

$\left\|G_{j}\right\|_{\delta \beta_{j}, \mu, \theta}:=\sum_{\nu=1 / 2, \mu}\left[\int_{\mathbb{R} \times S^{2}}\left\|\gamma_{\theta}\left(\frac{\sqrt{|u|+1}}{|u|^{\nu}} e^{\delta \beta_{j}|u| / 2} G_{j}\right)(u, \sigma)\right\|^{2} d u d \sigma\right]^{1 / 2}<\infty$,

for some fixed $\mu>1 / 2$ and where $\delta \beta_{j}=\beta-\beta_{j}$. For future references, $\theta_{0}:=\left(\delta_{0}, \tau_{0}\right)$.

(C) Non-degeneracy of the particle system. We have $\operatorname{dim} \mathcal{H}_{p}=N<\infty$ and the Hamiltonian $H_{p}$ has non-degenerate spectrum $\left\{E_{n}\right\}_{n=0}^{N-1}$.

(D) Fermi golden rule condition. We have, for $j=1,2$,

$$
\gamma_{0}:=\min _{0 \leq n<m \leq N-1} \int_{\mathbb{R}^{3}} \delta\left(|k|-E_{m n}\right)\left|G_{j}(k)_{n m}\right|^{2} d^{3} k>0,
$$

where $E_{m n}=E_{m}-E_{n}, G_{j}(k)_{m n}:=\left\langle\varphi_{m}, G_{j}(k) \varphi_{n}\right\rangle$, the $\varphi_{n}$ are normalized eigenvectors of $H_{p}$ corresponding to the eigenvalues $E_{n}$, and $\delta$ is the Dirac delta distribution.

(E) Either $\operatorname{dim} \mathcal{H}_{p}=2$, or $\operatorname{dim} \mathcal{H}_{p} \geq 3$ and the inverse temperatures satisfy

$$
\left|\beta_{1}-\beta_{2}\right| \leq c, \quad \text { or } \quad\left|\beta_{1}-\beta_{2}\right| \geq C \quad \text { and } \quad \min _{j} \beta_{j} \geq C
$$

where $0<c, C<\infty$ are constants depending only on the interaction $G_{1,2}$.

Remarks. 1) The map (3.1) has the following origin. In the positive-temperature representation of the CCR (the Araki-Woods representation on a suitable Hilbert space, see Appendix A), the interaction term $v_{j}$ is represented by $a_{j}\left(\widetilde{\gamma}_{\beta_{j}} G_{j}\right)+$ $a_{j}^{*}\left(\widetilde{\gamma}_{\beta_{j}} G_{j}\right)$, where

$$
\widetilde{\gamma}_{\beta}:=\sqrt{\frac{u}{1-e^{-\beta u}}} \gamma .
$$

2) The Condition (A) can be removed at expense of a slightly more involved proof of existence of the operator $\Gamma(z)$ in Lemma 4.2, see the remark after the proof of Lemma 4.2. However, since the ultraviolet behavior of the coupling functions is inessential in the models considered, we choose a stronger assumption over extra steps in the corresponding proof.

3) A class of interactions satisfying Conditions (A) and (B) is given by $G_{j}(k)=g(|k|) G$, where $g(u)=u^{\alpha} e^{-u^{2}}$, with $u \geq 0, \alpha=n+1 / 2, n=0,1,2, \ldots$, 
and $G=G^{*} \in \mathcal{B}\left(\mathcal{H}_{p}\right)$. A straightforward estimate gives that the norms (3.4) have the bound

$$
\left\|G_{j}\right\|_{\delta \beta_{j}, \mu, \theta} \leq C\left(1+e^{\left(\delta \beta_{j}\right)^{2} / 4}\right)\|G\|
$$

provided $\mu<\alpha+1$, where the constant $C$ does not depend on the inverse temperatures, nor on $\theta$ varying in any compact set (compare this with the bound (4.13) of [21]). The restriction $\alpha=n+1 / 2$ with $n=0,1,2, \ldots$ comes from the requirement of translation analyticity (the $\tau$-component of $\theta$ ), which appears also in [21].

4) The condition $\tau_{0} / \cos \delta_{0}<2 \pi / \beta$ after (3.3) guarantees that the square root in (3.7) is analytic in translations $u \mapsto u+\tau$.

5) What we need in our analysis is that the level shift operator $\Lambda_{0}$, the $N \times N$ matrix defined in (7.1), has a spectral gap at zero which is bounded below by a strictly positive constant independent of the temperatures. Condition (E) ensures this property. If one can show the desired property of the gap by other means then Condition (E) can be dropped.

Let

$$
\sigma:=\min \left\{|\lambda-\mu| \mid \lambda, \mu \in \sigma\left(H_{p}\right), \lambda \neq \mu\right\}
$$

and define

$$
g_{0}:=C \sigma^{1 / 2} \sin \left(\delta_{0}\right)\left[\left(1+\beta_{1}^{-1 / 2}+\beta_{2}^{-1 / 2}\right) \max _{j} \sup _{|\theta| \leq \theta_{0}}\left\|G_{j}\right\|_{\delta \beta_{j}, 1 / 2, \theta}\right]^{-1},
$$

where $C$ is a constant depending only on $\tan \delta_{0}$, and $\delta \beta_{j}=\beta-\beta_{j}, \delta \beta_{p}=\left|\beta-\beta_{p}\right|$, and set

$$
g_{1}:=\min \left(\left(g_{0}\right)^{1 / \alpha},\left[\min \left(\beta_{1}^{-1}, \beta_{2}^{-1}\right)\right]^{\frac{1}{2+\alpha}}\right),
$$

where $\alpha=\frac{\mu-1 / 2}{\mu+1 / 2}$, and $\mu>1 / 2$ is given in (3.4).

The main results of this paper are given in the following theorems, where by a "state" on a subalgebra (which is not necessarily a $C^{*}$-subalgebra), we mean a positive normalized linear functional.

Theorem 3.1. Assume conditions (A)-(E) are obeyed for some $0<\beta_{1}, \beta_{2}<\infty$, $\mu>1$, and let $\beta=\max \left(\beta_{1}, \beta_{2}\right)$.

If $0<|g|<g_{1}$ then there is a stationary state $\eta=\eta_{\beta_{1} \beta_{2}}$, defined on a strongly dense subalgebra $\mathcal{A}_{1}$ of $\mathcal{A}($ see $(2.7))$, satisfying

$$
\psi^{t} \rightarrow \eta, \quad t \rightarrow \infty
$$

for any $\beta_{1} \beta_{2}$-normal initial state $\psi . \eta$ is continuous in the norm of $\mathcal{A}$. The convergence is in the weak* sense (i.e., pointwise for each $\left.A \in \mathcal{A}_{1}\right)$. For $A \in \mathcal{A}_{1}, \eta(A)$ is analytic in $g$.

Remark. 6) Our analysis shows that the NESS is actually defined on a bigger (but somewhat less explicit) Banach space of operators $\mathcal{A}_{0} \supseteq \mathcal{A}_{1}$ (see (9.11)), and the convergence to the NESS, (3.11), holds on $\mathcal{A}_{0}$. On $\mathcal{A}_{1}$ one can introduce a "deformation norm" $\||\cdot|\| \leq\|\cdot\|$, see (9.9), such that in this norm, the convergence in (3.11) is uniform, $\sup _{A \in \mathcal{A}_{1}}\left|\psi^{t}(A)-\eta(A)\right| /|||A| \| \mid \rightarrow$. Moreover, on $\mathcal{A}_{1}$, the 
convergence is exponentially fast for initial conditions $\psi$ in a dense set (in the topology of bounded linear functionals on $\mathcal{A}$ ) - this set is the convex hull of vector states with deformation analytic vectors.

Theorem 3.2. Assume that the conditions of Theorem 3.1 are satisfied and let $\eta_{\beta_{1} \beta_{2}}$ and $g$ be as in Theorem 3.1. Let $\beta_{1} \neq \beta_{2}$, and let $g$ and $\left|\beta_{1}-\beta_{2}\right|$ be sufficiently small (independently). If either $G_{1}=G_{2}$ or $\operatorname{dim} \mathcal{H}_{p}=2$, then $\operatorname{EP}\left(\eta_{\beta_{1} \beta_{2}}\right)>0$.

Our analysis gives a stronger result than the one presented in Theorem 3.2. Namely, for $\mu>3 / 2$, we show that $E P\left(\eta_{\beta_{1} \beta_{2}}\right)>0$, provided $o\left(g^{0}\right) O(\delta \beta) \leq \eta^{\prime}$, where $\delta \beta=\left|\beta_{1}-\beta_{2}\right|$ (see Theorem 12.1). Here, $\eta^{\prime}$ depends on the inverse temperatures and the coupling functions and is given by

$$
\eta^{\prime}=\frac{2 \pi}{\sqrt{N}} \sum_{j>i}\left(\gamma_{j} e^{\beta_{1} E_{j i}}-\gamma_{i}\right) \frac{E_{j i} g_{j i}\left(E_{j i}\right)^{2}}{e^{\beta_{1} E_{j i}}-1},
$$

where $E_{j i}=E_{j}-E_{i}, g_{j i}(E)^{2}=\int_{\mathbb{R}^{3}} d^{3} k\left|\left\langle\varphi_{i}, G_{1}(k) \varphi_{j}\right\rangle\right|^{2} \delta\left(E_{j i}-\omega\right)$ (see Condition (C)). The numbers $\gamma_{j} \geq 0$ are the coordinates (in the basis $\left\{\varphi_{j} \otimes \varphi_{j}\right\}$ of $\left.\operatorname{Null}\left(L_{p}\right)\right)$ of the unique vector $\zeta^{*}$ in the kernel of the adjoint level shift operator $\Lambda_{0}^{*}$, at the value $\beta_{p}=0$ (and normalized as $\sum_{j} \gamma_{j}=\sqrt{N}$ ). (The operator $\Lambda_{0}$ is defined in Section 10.)

By general arguments one can prove that $\eta^{\prime} \geq 0$ for $\beta_{1}>\beta_{2}, \eta^{\prime} \leq 0$ for $\beta_{1}<\beta_{2}$, and $\eta^{\prime}=0$ if $\beta_{1}=\beta_{2}$. We also show that $\eta^{\prime}>0$ for $\beta_{1}>\beta_{2}$ and $\eta^{\prime}<0$ for $\beta_{1}<\beta_{2}$, for all $\beta_{1}, \beta_{2}$, except possibly for finitely many values in any compact set, see the remark at the end of Section 12 .

The dependence of $\eta^{\prime}$ on $\delta \beta$ is determined by the coordinates $\gamma_{j}$. We compute those in the cases when $G_{1}=G_{2}$ and $\operatorname{dim} \mathcal{H}_{p}=2$ (see the proof of Theorem 12.1, and equation (11.22), respectively).

Remarks. 7) Using Araki's theory of perturbation of KMS states, one shows that if the temperatures of both reservoirs are equal then the limit state is an equilibrium state and has zero entropy production. Non-existence of equilibrium states for $\beta_{1} \neq \beta_{2}$ has been shown in [25].

8) For a model with fermionic reservoirs, using a sufficiently fast convergence rate in (3.11) (e.g., $O\left(t^{-\alpha}\right)$ with $\alpha>1$ suffices) and the fact that $\eta$ is not a normal state for $\beta_{1} \neq \beta_{1}$, it has been shown by an abstract argument that $\operatorname{EP}\left(\eta_{\beta_{1} \beta_{2}}\right)>0$, provided $\left|\beta_{1}-\beta_{2}\right| \geq C g$ for some $C>0$ (see [21]). Instead of this indirect derivation we compute $\operatorname{EP}\left(\eta_{\beta_{1} \beta_{2}}\right)$ to the leading order in $g$ and derive the results stated in the theorem.

9) The condition $G_{1}=G_{2}$ can be relaxed to $G_{1}-G_{2}$ being small in a suitable sense.

\section{Spectral theory of NESS}

In this section we outline a spectral theory of NESS applicable to Bose and Fermi reservoirs. Our approach follows the one developed for the Fermi reservoirs in [21]. Fix a state, $\omega_{0}$, of the form $(2.10)$ with $\omega_{p}(A):=\operatorname{Tr}\left(e^{-\beta_{p} H_{p}} A\right) / \operatorname{Tr}\left(e^{-\beta_{p} H_{p}}\right)$. 
In this and the next section we use Conditions (A) and

$$
\left(H_{p}+i\right)^{-1} \text { is of a trace class. }
$$

In particular, the $\theta$-analyticity of the coupling functions and the finiteness of the dimension of the particle space are not required.

We pass to the Araki-Woods GNS representation for the unperturbed system:

$$
\left(\mathcal{A}, \omega_{0}\right) \rightarrow\left(\mathcal{H}, \pi, \Omega_{0}\right)
$$

where $\mathcal{H}, \pi$ and $\Omega_{0}$ are a Hilbert space, a representation of the algebra $\mathcal{A}$ by bounded operators on $\mathcal{H}$, and a cyclic element in $\mathcal{H}$ s.t.

$$
\omega_{0}(A)=\left\langle\Omega_{0}, \pi(A) \Omega_{0}\right\rangle .
$$

There is also an anti-linear representation, $\pi^{\prime}$, of the algebra $\mathcal{A}$ in bounded operators on the space $\mathcal{H}$, s.t. $\pi^{\prime}$ commutes with $\pi$ (i.e., $\left[\pi^{\prime}(A), \pi(B)\right]=0 \forall A, B \in \mathcal{A}$ ) and $\overline{\pi^{\prime}(\mathcal{A}) \Omega_{0}}=\mathcal{H}$.

The full dynamics is implemented by a one-parameter group $U(t)$ satisfying

$$
U(t) B \Omega=\sigma^{t}(B) \Omega, \quad \forall B \in \pi(\mathcal{A}),
$$

where $\Omega$ is a cyclic and separating vector for $\pi(\mathcal{A})$ to be specified below and where

$$
\sigma^{t}(B):=e^{i t L^{(\ell)}} B e^{-i t L^{(\ell)}}
$$

for any $B \in \pi(\mathcal{A})$ with, recall, $L^{(\ell)}:=L_{0}+g \pi(v)$ and $L_{0}$ is defined on the line before (2.26) (see also the paragraph after (2.37)). Observe that (4.2) implies that

$$
U(t) \Omega=\Omega .
$$

If the state $\omega$ corresponding to $\Omega$ is stationary (i.e., $\left.\omega^{t}=\omega\right)$ then $U(t)$ comes out to be unitary. In our situation we expect that there is no $\omega_{0}$-normal stationary state and $U(t)$ will be a non-unitary group.

We pick the vector $\Omega$ as follows. Let $\beta=\max _{j=1,2} \beta_{j}$. We define

$$
\Omega:=e^{-\beta L^{(\ell)} / 2} \Omega_{0} /\left\|e^{-\beta L^{(\ell)} / 2} \Omega_{0}\right\| .
$$

The facts that $\Omega$ is well defined, i.e., that $\Omega_{0} \in \operatorname{Dom}\left(e^{-\beta L^{(\ell)} / 2}\right)$, and that $\Omega$ is cyclic and separating, are established in Proposition 4.1 at the end of this section.

The family $U(t)$ is not unitary since $\omega:=\langle\Omega, \pi(\cdot) \Omega\rangle$ is not stationary:

$$
\begin{aligned}
\langle U(t) \pi(A) \Omega, U(t) \pi(B) \Omega\rangle & =\left\langle\Omega, \sigma^{t}\left(\pi\left(A^{*} B\right)\right) \Omega\right\rangle=\omega^{t}\left(A^{*} B\right) \\
& \neq \omega\left(A^{*} B\right)=\langle\pi(A) \Omega, \pi(B) \Omega\rangle,
\end{aligned}
$$

for some $A, B, t$. Let now $\psi$ be an $\omega_{0}$-normal state corresponding to the vector $Q \Omega \in \mathcal{H}$ (i.e., $\psi(A)=\langle Q \Omega, \pi(A) Q \Omega\rangle$ ), where $Q \in \pi^{\prime}(\mathcal{A})$ then

$$
\psi^{t}(A)=\left\langle Q \Omega, \sigma^{t}(\pi(A)) Q \Omega\right\rangle .
$$


Due to (A.4) and $\sigma_{0}^{t}(\pi(A)):=\pi\left(\alpha_{0}^{t}(A)\right)$ and due to convergence of $\sigma_{(n)}^{t}(\pi(A))$ to $\sigma^{t}(\pi(A))$ established after (A.4), we see that the operator $Q$ commutes with $\sigma^{t}(\pi(A))$. Using this together with (4.2) and (4.4) we arrive at

$$
\psi^{t}(A)=\left\langle Q^{*} Q \Omega, U(t) \pi(A) \Omega\right\rangle .
$$

This key formula, due to [21], connects the long time behaviour of $\psi^{t}(A)$ with spectral properties of $U(t)$ or its generator. We explain what this means.

Assume we can show that, for a certain class of $\phi$ and $\Psi$, and as $t \rightarrow \infty$,

$$
\langle\phi, U(t) \Psi\rangle \rightarrow\langle\phi, P \Psi\rangle
$$

where $P$ is the eigenprojection on the fixed point subspace of $U(t)$ (i.e., $U(t) P=$ $P U(t)=P)$, which we assume for a moment to exist. Relations (4.8) and (4.9) imply

$$
\psi^{t} \rightarrow \eta, \quad t \rightarrow \infty,
$$

where the state $\eta$ is defined (on an appropriate set of observables) by

$$
\eta(A):=\left\langle Q^{*} Q \Omega, P \pi(A) \Omega\right\rangle .
$$

We will show below that (4.9) holds for some unbounded projection operator $P$. To understand the structure of this operator, we proceed as follows.

We will show that $U(t)$ is strongly differentiable on a dense set and we will compute its non-self-adjoint generator, $K:=-\left.i \frac{\partial}{\partial t}\right|_{t=0} U(t)$, which satisfies (see $(4.4))$

$$
K \Omega=0 .
$$

The operator $P$ is the eigenprojection onto the eigenspace of $K$ associated with the eigenvalue 0 (i.e., $K P=P K=0$ ). We show that $\operatorname{dim} P=1$ and

$$
P=|\Omega\rangle\left\langle\Omega^{*}\right|
$$

for some $\Omega^{*} \notin \mathcal{H}$, satisfying $K^{*} \Omega^{*}=0$ in a weak sense $\left(\Omega^{*} \in \mathcal{D}_{\text {anal }}^{\prime}\right.$, where $\mathcal{D}_{\text {anal }}=\cup_{\operatorname{Im} \theta>0} \operatorname{Dom}\left(U_{\theta}\right)$ with the family $U_{\theta}$ defined in Section 6). Understanding the nature of the vector $\Omega^{*}$, which we call the NESS vector, is a goal of our analysis.

Substituting (4.13) into (4.11) and using that $\left\langle Q^{*} Q \Omega, \Omega\right\rangle=\|Q \Omega\|^{2}=\psi(\mathbf{1})=1$, we obtain

$$
\eta(A)=\left\langle\Omega^{*}, \pi(A) \Omega\right\rangle .
$$

Since $\Omega^{*} \notin \mathcal{H}$ the state $\eta$ is not normal but it is well defined for a dense set of observables. The question now is what is $\Omega^{*}$ ? The answer, provided in subsequent sections, is that $\Omega^{*}$ is a resonance of $K^{*}$.

In the following sections we construct a mathematical framework which provides meaningful expressions replacing formal ones, (4.9)-(4.14), and with the help of which we can prove the convergence (4.10).

Remark. Evolution groups and their generators given by conditions of the type of (4.4) (or (4.12)) were introduced in [21], where the group $U(t)$ is specified by the condition $U(t) \Omega_{0}=\Omega_{0}$. where $\Omega_{0}$ is the unperturbed vector ("vacuum") 
introduced in (2.37) above. However, an analysis of the operator $K$ (see Sections 6 and 7 ) defined this way requires, instead of Condition (B), the condition obtained from Condition (B) by replacing the weight $e^{\delta \beta_{j}|u| / 2}$ by $e^{\beta_{j}|u| / 2}$. This leads to an additional restriction on the temperatures of the form

$$
g \leq c \min _{j}\left\{e^{-\beta_{j}}\right\}, \quad \text { i.e., } \quad \min _{j} T_{j} \geq c[\ln (1 / g)]^{-1} .
$$

Using in [21] the vector $\Omega$ instead of $\Omega_{0}$ would improve this bound to $\min _{j} T_{j} \geq c g$.

Now we proceed to the main technical result of this section - the proof of the existence of the vector $\Omega$ and establishing its properties mentioned and used above.

Proposition 4.1. $\Omega_{0} \in \operatorname{Dom}\left(e^{-\beta L^{(\ell)} / 2}\right)$ and the vector $\Omega:=e^{-\beta L^{(\ell)} / 2} \Omega_{0} /\left\|e^{-\beta L^{(\ell)} / 2} \Omega_{0}\right\|$ is cyclic and separating for the von Neumann algebra $\pi(\mathcal{A})^{\prime \prime}$.

We begin with some preliminary technical results. To do manipulations with unbounded operators we use the dense subset, $\mathcal{D}$, of our Hilbert space, $\mathcal{H}$, defined by

$$
\mathcal{D}:=\pi(\tilde{\mathcal{A}}) \Omega_{0},
$$

where $\tilde{\mathcal{A}}=\mathcal{B}\left(\mathcal{H}_{p}\right) \otimes \mathcal{P}_{1} \otimes \mathcal{P}_{2}$. Here $\mathcal{P}_{j}$ is the polynomial algebra generated by the annihilation and creation operators, $a_{j}(f)$ and $a_{j}^{*}(f)$, of the $j$-th reservoir acting on $\mathcal{H}_{r j}$ with $f \in L^{2}\left(\mathbb{R}^{3}, e^{a^{\prime}|k|^{2}}\left(1+|k|^{-1}\right) d^{3} k\right)$ for some $a^{\prime}>0$. $\mathcal{D}$ is a subset of

$$
\mathcal{F}_{0}:=\left\{\psi \in \mathcal{H} \mid P_{n} \psi=\psi, \text { for some } n<\infty\right\},
$$

where $P_{n}$ is the spectral projection of the self-adjoint operator $\widetilde{N}=\max \left\{N_{1}, N_{2}\right\}:=$ $\int \max (\lambda, \mu) d E_{N_{1}}(\lambda) \otimes d E_{N_{2}}(\mu)$ associated to the interval $[0, n]$. Here, $N_{j}:=$ $\int\left[a_{\ell, j}^{*}(k) a_{\ell, j}(k)+a_{r, j}^{*}(k) a_{r, j}(k)\right] d^{3} k$ is the number operator of reservoir $j$ (see also $(6.3)) . \mathcal{F}_{0}$ is commonly called the finite-particle subspace.

Let $\Delta$ and $J$ be the modular operator and modular conjugation associated with the pair $\left\{\pi(\mathcal{A})^{\prime \prime}, \Omega_{0}\right\}$ and let $\kappa^{t}$ be defined by

$$
\kappa^{t}=\alpha_{p}^{\beta_{p} t} \otimes \alpha_{r 1}^{\beta_{1} t} \otimes \alpha_{r 2}^{\beta_{2} t} .
$$

The vector $\Omega_{0}$ defines a $(\kappa, 1)$-KMS state and one can show that

$$
\Delta^{-i t} A \Delta^{i t}=\pi\left(\kappa^{t}(A)\right) \quad \forall A \in \mathcal{A} .
$$

Using this one computes that $\Delta=e^{-\tilde{L}}$, where $\tilde{L}=\beta_{p} L_{p}+\beta_{r 1} L_{r 1}+\beta_{r 2} L_{r 2}$, and, in particular, $\Delta^{i t}$ commutes with $e^{i t L_{0}}$. Observe that

$$
e^{\tau L_{0}} \mathcal{D}=\mathcal{D} \quad \text { and } \quad \Delta^{\tau} \mathcal{D}=\mathcal{D} \quad \forall \tau \in \mathbb{R} .
$$

In the sequel, an important role is played by the operators

$$
\Gamma(z):=e^{-z L^{(\ell)} / 2} e^{z L_{0} / 2}
$$

(defined as products of two, in general, unbounded operators). We study properties of these operators in the following lemma. 
Lemma 4.2. The following statements hold for all $z \in \mathbb{C}$ and all $\varphi, \psi \in \mathcal{D}$ :

$$
\begin{aligned}
& \mathcal{D} \subset \operatorname{Dom}(\Gamma(z)), \\
& z \mapsto \Gamma(z) \varphi \quad \text { is entire, } \\
& e^{-z L^{(\ell)} / 2}=\Gamma(z) e^{-z L_{0} / 2} \quad \text { on } \mathcal{D}, \\
& \langle B \Gamma(\bar{z}) \psi, \Gamma(-z) \varphi\rangle=\langle B \psi, \varphi\rangle \quad \forall B \in \pi(\mathcal{A})^{\prime}, \\
& \left\langle J A \Gamma(z) \Omega_{0}, \varphi\right\rangle=\left\langle A^{*} \Omega_{0}, \Gamma(z) \Delta^{1 / 2} \varphi\right\rangle, \quad \forall A \in \pi(\mathcal{A})^{\prime \prime}, \\
& \Gamma(z) \mathcal{D} \quad \text { is dense. }
\end{aligned}
$$

Remark. $\pi(\mathcal{A})^{\prime}$ is the von Neumann algebra $\pi^{\prime}(\mathcal{A})^{\prime \prime}$, the weak closure of $\pi^{\prime}(\mathcal{A})$.

Proof of Proposition 4.1. Let $\Gamma=\Gamma(\beta)$, where $\Gamma(z)$ is defined in (4.21). Since $e^{\tau L_{0}} \Omega_{0}=\Omega_{0}$, the property $\Omega_{0} \in \operatorname{Dom}\left(e^{-\beta L^{(\ell)} / 2}\right)$ is equivalent to $\Omega_{0} \in \operatorname{Dom}(\Gamma)$ (see $(4.24)$ ), which is proven in Lemma 4.2, relation (4.22). Hence the vector $\Omega$ exists.

For the cyclicity it is enough to show that if $B \in \pi(\mathcal{A})^{\prime}$ and $B \Omega=0$ then $B=0$. Let $\Omega^{\prime}:=e^{-\beta L^{(\ell)} / 2} \Omega_{0}=\Gamma \Omega_{0}$. By $(4.25), \forall \varphi \in \mathcal{D}, 0=\left\langle B \Gamma(\beta) \Omega_{0}, \Gamma(-\beta) \varphi\right\rangle=$ $\left\langle B \Omega_{0}, \varphi\right\rangle$. This implies $B \Omega_{0}=0$. Since $\Omega_{0}$ is separating for $\pi(\mathcal{A})^{\prime}$ we have that $B=0$.

Now we show that $\Omega$ is separating for $\pi(\mathcal{A})^{\prime \prime}$. Let $A \in \pi(\mathcal{A})^{\prime \prime}$ be such that $A \Omega=0$. The relation $A \Gamma \Omega_{0}=0$ and equation (4.26) imply that

$$
0=\left\langle J A \Gamma \Omega_{0}, \varphi\right\rangle=\left\langle A^{*} \Omega_{0}, \Gamma \Delta^{1 / 2} \varphi\right\rangle,
$$

for any $\varphi \in \mathcal{D} \subset \mathcal{F}_{0}$. Now $\Delta^{1 / 2} \mathcal{D}=\mathcal{D}$ and $\Gamma \mathcal{D}$ is dense (as is shown in (4.27)), so we have that $\Gamma \Delta^{1 / 2} \mathcal{D}$ is dense and it follows from (4.28) that $A^{*} \Omega_{0}=0$. Since $\Omega_{0}$ is separating this implies that $A=0$ and therefore $\Omega$ is separating.

Proof of Lemma 4.2. We first show that for all $\varphi \in \mathcal{F}_{0}$ the following formal Dyson expansion of the operator $\Gamma(z)$ is well defined:

$$
\tilde{\Gamma}(z) \varphi:=\sum_{m \geq 0}\left(\frac{-g z}{2}\right)^{m} \int_{0}^{1} d \tau_{1} \cdots \int_{0}^{\tau_{m-1}} d \tau_{m} \sigma_{0}^{i z \tau_{m} / 2}(V) \cdots \sigma_{0}^{i z \tau_{1} / 2}(V) \varphi .
$$

The integrals on the r.h.s. are understood as strong limits of Riemann sums. Due to the UV-cut-off Condition (A), the transformation $\sigma_{0}^{i w}(V)=e^{-w L_{0}} V e^{w L_{0}}$ is well defined and strongly analytic on $\mathcal{F}_{0}$ for $w \in \mathbb{C}$. Since $\varphi \in \mathcal{F}_{0}$, there is a $\nu_{0}$ s.t. $P_{\nu_{0}} \varphi=\varphi$ (see also (4.17)). Because each interaction $V$ can increase the number of particles in each reservoir by at most one, we can write the integrand of (4.29) as

$$
\sigma_{0}^{i z \tau_{m} / 2}(V) P_{\nu_{0}+m-1} \sigma_{0}^{i z \tau_{m-1} / 2}(V) P_{\nu_{0}+m-2} \cdots \sigma_{0}^{i z \tau_{1} / 2}(V) P_{\nu_{0}} \varphi
$$

Since $\sigma_{0}^{i w}(V) P_{k}$ are bounded operators, the integrand on the r.h.s. of (4.29) belong to $\mathcal{H}$. Moreover, it is strongly continuous in $\tau_{1}, \ldots, \tau_{m}$. 
Let us first show that the series in (4.29) converges absolutely, for all values of $g, z \in \mathbb{C}$. We use the bound $\left\|\sigma_{0}^{i w}(V) P_{\nu}\right\| \leq C(\operatorname{Im} w)(\nu+1)^{1 / 2}$ which follows in a standard way from the explicit expression of $V$. The constant is given by

$$
C(\operatorname{Im} w)=2 e^{2 \operatorname{Im} w\left\|H_{p}\right\|} \sum_{j=1,2}\left[\int_{\mathbb{R}^{3}}\left\|G_{j}(k)\right\|^{2} \frac{e^{|k|\left(\operatorname{Im} w+\beta_{j}\right)}}{e^{\beta_{j}|k|}-1} d^{3} k\right]^{1 / 2} .
$$

It follows that the norm of the $m$-th term in the series of (4.29) has the upper bound $\frac{[|g z| C(\operatorname{Im} z / 2)]^{m}}{2^{m} m !} \sqrt{\frac{\left(\nu_{0}+m\right) !}{\nu_{0} !}}$. Consequently, the series converges absolutely, for any $g, z \in \mathbb{C}$, and for any $\varphi \in \mathcal{F}_{0}$.

Next, we show that $\mathcal{D} \subset \operatorname{Dom}(\Gamma(z))$, and that $\tilde{\Gamma}(z) \varphi=\Gamma(z) \varphi$ for all $\varphi \in \mathcal{D}$. It suffices to establish that for any $\varphi \in \mathcal{D}$,

$$
\left\langle e^{-\bar{z} L^{(\ell)} / 2} \psi, e^{z L_{0} / 2} \varphi\right\rangle=\langle\psi, \tilde{\Gamma}(z) \varphi\rangle,
$$

for all $\psi \in \mathcal{H}$ s.t. $\psi=f\left(L^{(\ell)}\right) \psi$ for some $f \in C_{0}^{\infty}(\mathbb{R})$ (such $\psi$ form a core for $\left.e^{-\bar{z} L^{(\ell)} / 2}\right)$. Indeed, this would show that $e^{z L_{0} / 2} \varphi \in \operatorname{Dom}\left(e^{-z L^{(\ell)} / 2}\right)$ and therefore $\varphi \in \operatorname{Dom}(\Gamma(z))$. Equation (4.30) can be shown, e.g., using the analyticity of both sides in $z$, and the fact that the equation holds for $z \in i \mathbb{R}$. Indeed, in the latter case $\Gamma(z)$ are bounded operators and the Dyson series expansion (4.29) is valid for them. In particular, $\mathcal{D} \subset \operatorname{Dom}(\Gamma(z))$. Moving $e^{-\bar{z} L^{(\ell)} / 2}$ in (4.30) to the right factor proves that $\Gamma(z) \varphi=\tilde{\Gamma}(z) \varphi$, for all $\varphi \in \mathcal{D}$. This also shows (4.23), since the series (4.29) is entire in $z$. Thus we have shown (4.22) and (4.23).

Furthermore, since $e^{z L_{0} / 2} \mathcal{D}=\mathcal{D}$, we have by the argument above (see (4.30) and the argument after it) that (4.24) holds. Moreover, $\forall \varphi \in \mathcal{D}, e^{-z L^{(\ell)} / 2} \varphi$ is analytic due to the formula $e^{-z L^{(\ell)} / 2} \varphi=\Gamma(z) e^{-z L_{0} / 2} \varphi$ and analyticity of the factors on the r.h.s. .

To prove (4.25) we note that, due to the Dyson expansion, it is true for purely imaginary $z$ and by analyticity of the l.h.s. for all $z$.

Now, we prove (4.26). Denote by $\Gamma_{k}(z) \varphi$ the truncated series on the r.h.s. of (4.29), with $m \leq k$. Let $A \in \pi(\mathcal{A})^{\prime \prime}$. Choose a sequence of linear combinations of $\left(\mathcal{H}_{p} \otimes \mathcal{H}_{p}\right.$-valued) field operators $A_{n} \in \pi(\widetilde{\mathcal{A}})$ converging weakly to $A$. We use the defining property of the operator $S=\Delta^{-1 / 2} J$, and the results proven above, to obtain the following relation for all $\varphi \in \mathcal{D}$ :

$$
\begin{aligned}
\left\langle J A \Gamma(z) \Omega_{0}, \varphi\right\rangle & =\lim _{k \rightarrow \infty}\left\langle J A \Gamma_{k}(z) \Omega_{0}, \varphi\right\rangle \\
& =\lim _{k \rightarrow \infty} \lim _{n \rightarrow \infty}\left\langle J A_{n} \Gamma_{k}(z) \Omega_{0}, \varphi\right\rangle \\
& =\lim _{k \rightarrow \infty} \lim _{n \rightarrow \infty}\left\langle\Gamma_{k}(z)^{*}\left(A_{n}\right)^{*} \Omega_{0}, \Delta^{1 / 2} \varphi\right\rangle \\
& =\lim _{k \rightarrow \infty}\left\langle A^{*} \Omega_{0}, \Gamma_{k}(z) \Delta^{1 / 2} \varphi\right\rangle \\
& =\left\langle A^{*} \Omega_{0}, \Gamma(z) \Delta^{1 / 2} \varphi\right\rangle .
\end{aligned}
$$


We have used that $\left(A_{n}\right)^{*} \Omega_{0} \in \mathcal{D} \subset \mathcal{F}_{0}, \Delta^{1 / 2} \psi \in \mathcal{D} \subset \mathcal{F}_{0}$, and in (4.31) that $J \Delta^{1 / 2} A_{n} \Gamma_{k}(z) \Omega_{0}=\Gamma_{k}(z)^{*}\left(A_{n}\right)^{*} \Omega_{0}$. The latter relation follows from the facts that $\Gamma_{k}(z)$ is affiliated with the von Neumann algebra $\pi(\mathcal{A})^{\prime \prime}$, and that $\left(A_{n}\right)^{*}$ leaves $\mathcal{F}_{0}$ invariant. It can also be verified directly, using the explicit actions of $J$ and $\Delta^{1 / 2}$. This shows (4.26).

To prove the last statement we introduce a new family of operators $\Gamma^{\&}(z):=$ $e^{z L_{0} / 2} e^{-z L^{(\ell)} / 2}$ related to the adjoint of $\Gamma(z)$. First we prove that $\mathcal{D} \subset \operatorname{Dom}\left(\Gamma^{\&}(z)\right)$. To this end we note that, exactly as above, the formal expansion of $\Gamma^{\&}(z)$, which we denote by $\tilde{\Gamma}^{\&}(z)$, converges on elements of $\mathcal{D}$ and is entire as a function of $z$. Next, we observe that, since $e^{z L_{0} / 2} \mathcal{D}=\mathcal{D}$, the equation $e^{z L_{0} / 2} \varphi \in \operatorname{Dom}\left(e^{-z L^{(\ell)} / 2}\right)$, proven above, implies that $\mathcal{D} \subset \operatorname{Dom}\left(e^{-z L^{(\ell)} / 2}\right)$. Let $\mathcal{D}_{1}:=\left\{\psi \in \mathcal{H} \mid \psi=f\left(L_{0}\right) \psi\right.$ for some $\left.f \in C_{0}^{\infty}(\mathbb{R})\right\}$. Then $\mathcal{D}_{1}$ is a core for $e^{-z L_{0} / 2}$. Now we claim that for any $\varphi \in \mathcal{D}$ and any $\psi \in \mathcal{D}_{1}$,

$$
\left\langle e^{\bar{z} L_{0} / 2} \psi, e^{-z L^{(\ell)} / 2} \varphi\right\rangle=\left\langle\psi, \tilde{\Gamma}^{\&}(z) \varphi\right\rangle .
$$

Indeed, the latter relation is true for $z$ purely imaginary and it remains to be true for complex $z$ by analyticity of both sides. The last relation and the fact that $\mathcal{D}_{1}$ is a core of the operator $e^{\bar{z} L_{0} / 2}$ show that $e^{-z L^{(\ell)} / 2} \varphi \in \operatorname{Dom}\left(e^{z L_{0} / 2}\right)$ and $\left\langle\psi, e^{z L_{0} / 2} e^{-z L^{(\ell)} / 2} \varphi\right\rangle=\left\langle\psi, \tilde{\Gamma}^{\&}(z) \varphi\right\rangle$. In other words, $\varphi \in \operatorname{Dom}\left(\Gamma^{\&}(z)\right)$ and $\Gamma^{\&}(z) \varphi=\tilde{\Gamma}^{\&}(z) \varphi$. This proves that $\mathcal{D} \subset \operatorname{Dom}\left(\Gamma^{\&}(z)\right)$ and that $\Gamma^{\&}(z)$ is an entire operator-function.

To prove (4.27) it suffices to show that $\Gamma(z) \mathcal{D} \supseteq \mathcal{D}$. To prove the latter, let $\psi \in \mathcal{D} \subset \operatorname{Dom}\left(\Gamma^{\&}(z)\right)$. Then $e^{z L_{0} / 2} e^{-z L^{(\ell)} / 2} \psi \in \operatorname{Dom}\left(e^{z L^{(\ell)} / 2} e^{-z L_{0} / 2}\right)$, and the image of this vector under $e^{z L^{(\ell)} / 2} e^{-z L_{0} / 2}$ is just $\psi$. Hence $\psi \in \Gamma(z) \mathcal{D}$ as required.

This completes the proof of Lemma 4.2 .

Remark. A sharper estimate can be obtained by using a standard argument to estimate the norms of the integrands (to keep notation simple we set $\varphi=\Omega_{0}$ ),

$$
\left\|\sigma_{0}^{i \tau_{m}}(V) \cdots \sigma_{0}^{i \tau_{1}}(V) \Omega_{0}\right\|=\omega_{0}\left(\alpha_{0}^{-i \tau_{1}}(v) \cdots \alpha_{0}^{-i \tau_{m}}(v) \alpha_{0}^{i \tau_{m}}(v) \cdots \alpha_{0}^{i \tau_{1}}(v)\right)^{1 / 2},
$$

by using Wick's theorem, the expression for the imaginary-time two-point functions, in the same way as it is done, for instance, in [8], Thm IV.3.

\section{Generator $K$ and interpolating family $K_{(s)}$}

In this section we find an explicit form and some properties of the generator $K$ of the one-parameter group $U(t)$ introduced in the preceding section (cf. [21]), and of the family $K_{(s)}$ which interpolates $K$ to a selfadjoint operator.

Let $\omega_{0}$ be the state of the algebra $\mathcal{A}$ fixed at the beginning of the Section 4 and let $J$ and $\Delta$ be the Tomita-Takesaki modular conjugation and modular operator associated with the couple $\left(\mathcal{A}, \omega_{0}\right)$. We have the following standard relations:

$$
J \pi(A) J=\pi^{\prime}(A),
$$


$J \Delta^{1 / 2} \pi(A) \Omega_{0}=\pi\left(A^{*}\right) \Omega_{0}, \Delta^{1 / 2} \Omega_{0}=\Omega_{0}$ and $\Delta^{-i t} \pi(A) \Delta^{i t}=\pi\left(\kappa^{t}(A)\right)$, where $\kappa$ is the automorphism of the algebra $\mathcal{A}$ defined in (4.18). The last three equations imply

$$
J \pi(A) \Omega_{0}=\pi\left(\kappa^{i / 2}\left(A^{*}\right)\right) \Omega_{0} .
$$

Finally, we recall that $\beta=\max \left(\beta_{1}, \beta_{2}\right)$ and that $L^{(\ell)}$ is the self-adjoint operator defined as $L^{(\ell)}:=L_{0}+g V$ where $V:=\pi(v)$.

Theorem 5.1. Assume that (A) and (4.1) hold. The semigroup $U(t)$, defined in (4.2)-(4.4), is differentiable on the domain $\operatorname{Dom}\left(L^{(\ell)}\right) \cap \pi(\mathcal{A}) \Omega$, and the generator $K=-\left.i \frac{\partial}{\partial t}\right|_{t=0} U(t)$ is given on this domain by the expression

$$
K=L_{0}+g\left(V-V_{-i / 2}^{\prime}\right),
$$

where $V=\pi(v)$ and $V_{s}^{\prime}=\pi^{\prime}\left(\gamma^{\bar{s}}(v)\right)$ with $\gamma^{s}:=\alpha_{0}^{-\beta s} \circ \kappa^{s}$ (due to condition (A) the operator $V_{-i / 2}^{\prime}$ is well defined). Furthermore, the domain $\operatorname{Dom}\left(L^{(\ell)}\right) \cap \pi(\mathcal{A}) \Omega$ is dense in $\mathcal{H}$.

Remark. The imaginary part of the generator $K$ is not semi-bounded. Therefore, the group $U(t)$, densely defined on $\pi(\mathcal{A}) \Omega$, does not extend to a group of bounded operators.

Proof of Theorem 5.1. Due to Condition (A), we have $e^{-\beta L_{0} / 2} L^{(\ell)} \Omega_{0}=$ $g e^{-\beta L_{0} / 2} \pi(v) \Omega_{0}=g \pi\left(\sigma_{0}^{i \beta / 2}(v)\right) \Omega_{0} \in \mathcal{D}$. It thus follows from Lemma 4.2 that $L^{(\ell)} \Omega_{0} \in \operatorname{Dom}\left(e^{-\beta L^{(\ell)} / 2}\right)$. The latter fact implies that $e^{i t L^{(\ell)}} \Omega$ is differentiable at $t=0$ and therefore $\Omega \in \operatorname{Dom}\left(L^{(\ell)}\right)$. Now, let $B \in \pi(\mathcal{A})$ be such that $B \Omega \in$ $\operatorname{Dom}\left(L^{(\ell)}\right)$. Taking into account equation $(4.3)$ we see that $\sigma^{t}(B)$ and therefore, due to (4.2), also $U(t) B \Omega$, are differentiable in $t$ at $t=0$. Indeed, let $\frac{1}{t}\left(e^{i t L^{(\ell)}} B e^{-i t L^{(\ell)}}-B\right) \Omega=F+G$, where $F:=e^{i t L^{(\ell)}} B \frac{1}{t}\left(e^{-i t L^{(\ell)}}-1\right) \Omega$ and $G:=\frac{1}{t}\left(e^{i t L^{(\ell)}}-1\right) B \Omega$. Clearly, $F \rightarrow-i B L^{(\ell)} \Omega$ and $G \rightarrow i L^{(\ell)} B \Omega$. Now, differentiating equation (4.2) we find

$$
K B \Omega=L^{(\ell)} B \Omega-B L^{(\ell)} \Omega .
$$

Now we compute the last term on the r.h.s. of this expression. To this end we use the following relations:

$$
\pi(A) \Omega_{0}=\pi^{\prime}\left(\kappa^{i / 2}\left(A^{*}\right)\right) \Omega_{0}
$$

and, for $z=i t$,

$$
e^{z L^{(\ell)}} \pi^{\prime}(A) e^{-z L^{(\ell)}}=\pi^{\prime}\left(\alpha_{0}^{i \bar{z}}(A)\right) .
$$

Equation (5.5) follows from relations (5.1) and (5.2). Equality (5.6) is proven by using the Kato-Trotter product formula.

Now, we claim that $\forall \psi \in \mathcal{D}$

$$
\left\langle\psi, B e^{z L^{(\ell)}} \pi(v) \Omega_{0}\right\rangle=\left\langle\left[\pi^{\prime}\left(\alpha_{0}^{i \bar{z}} \circ \kappa^{i / 2}(v)\right)\right]^{*} \psi, B e^{z L^{(\ell)}} \Omega_{0}\right\rangle .
$$

Observe that the vectors $e^{z L^{(\ell)}} \varphi=\Gamma(-2 z) e^{z L_{0}} \varphi, \varphi \in \mathcal{D}$, are entire in $z$, by Lemma 4.2. Hence both sides of (5.7) are entire in $z$. Therefore it suffices to 
prove (5.7) for $z=i t$. Equation (5.7) with $z=i t$ follows from the relations (5.5) and (5.6) proven above. Thus (5.7) is demonstrated.

Now, let $\Omega^{\prime}:=e^{-\beta L^{(\ell)} / 2} \Omega_{0}$. Recall that $L^{(\ell)} \Omega^{\prime}=g e^{-\beta L^{(\ell)} / 2} \pi(v) \Omega_{0}$. Then (5.7) with $z=\beta / 2$ and the definition of the transformation $\gamma^{s}$ imply that $\forall \psi \in \mathcal{D}$

$$
\begin{aligned}
\left\langle\psi, B L^{(\ell)} \Omega^{\prime}\right\rangle & =g\left\langle\psi, B e^{-\beta L^{(\ell)} / 2} \pi(v) \Omega_{0}\right\rangle \\
& =g\left\langle\left[\pi^{\prime}\left(\gamma^{i / 2}(v)\right)\right]^{*} \psi, B \Omega^{\prime}\right\rangle .
\end{aligned}
$$

This relation and the fact that $\mathcal{D}$ is a core of $\pi^{\prime}\left(\gamma^{i / 2}(v)\right)^{*}$ (which is a linear combination of creation and annihilation operators) show that $B \Omega^{\prime} \in \operatorname{Dom}\left(\pi^{\prime}\left(\gamma^{i / 2}(v)\right)\right.$ and

$$
B L^{(\ell)} \Omega=\pi^{\prime}\left(\gamma^{i / 2}(v)\right) B \Omega .
$$

Since the r.h.s. of (5.9) is $V_{-i / 2}^{\prime} B \Omega$, this equation together with (5.4) implies (5.3). Finally, $\operatorname{Dom}\left(L^{(\ell)}\right) \cap \pi(\mathcal{A}) \Omega$ contains the set $\mathcal{D}$ and is therefore dense in $\mathcal{H}$.

Observe that

$$
\left.\kappa^{t}\right|_{\beta_{j}=\beta_{p}=\beta}=\alpha_{0}^{\beta t} \text { and }\left.\quad \gamma^{t}\right|_{\beta_{j}=\beta_{p}=\beta}=\mathrm{id} .
$$

This implies that

$$
\left.K\right|_{\beta_{j}=\beta_{p}=\beta}=L,
$$

where $L:=L_{0}+g V-g V^{\prime}$, with $V^{\prime}:=\pi^{\prime}(v)$, is the standard self-adjoint Liouville operator. In what follows we write $K=L_{0}+g I$, where

$$
I=V-V_{-i / 2}^{\prime} .
$$

The operator $K$ is non-self-adjoint for $\delta \beta \neq 0$, and the perturbation $I$ is not relatively bounded w.r.t. the unperturbed operator $L_{0}$. To study the evolution generated by $K$ we use the family of operators

$$
K_{(s)}:=L_{0}+g\left(V-V_{s}^{\prime}\right),
$$

where, recall, $V=\pi(v)$, and $V_{s}^{\prime}:=\pi^{\prime}\left(\gamma^{\bar{s}}(v)\right)$. This family interpolates between the operator $K$,

$$
K=K_{(-i / 2)},
$$

(see (5.3)) and the self-adjoint operators $K_{(s)}$ with real $s$. Under condition (A) on $v, V_{(s)}^{\prime}$ and $K_{(s)}$ are well defined on the dense domain $\operatorname{Dom}\left(L^{(\ell)}\right) \cap \pi(\mathcal{A}) \Omega$ for all $s$ in the strip

$$
S_{\varepsilon}:=\left\{t \in \mathbb{C}|| \operatorname{Im} t \mid<\frac{1}{2}+\varepsilon\right\},
$$

for $\varepsilon>0$, and are strongly analytic there (recall that $\pi^{\prime}$ is anti-linear). 


\section{Spectral deformation of $K$ and $K_{(s)}$}

Since the operator $K$ is not self-adjoint it is not a simple matter to derive longtime properties of the dynamics $e^{i K t}$ from spectral properties of $K$. As a result we bypass establishing the connection of the dynamics to the spectrum of $K$ and instead connect it to certain spectral properties of a complex deformation, $K_{\theta}$, of this operator. To do this we use the interpolating family $K_{(s) \theta}$, which is the complex deformation of the family $K_{(s)},(5.12)$. In this section we define complex deformations $K_{\theta}$ and $K_{(s) \theta}$ and in the next section we establish their spectral characteristics which are relevant for us.

In order to carry out the spectral analysis of the operator $K$, which we begin in this section, we use the specifics of the Araki-Woods representation in an essential way. They were not used in an essential way for the developments up to this section.

As a complex deformation we choose a combination of the complex dilation used in [8] and complex translation due to [18] (see [8], Section V.2 for a sketch of the relevant ideas). This complex deformation was used in [25] in the spectral analysis for a general class of Liouville type operators.

First we define the group of dilations. Let $\hat{U}_{d, \delta}$ be the second quantization of the one-parameter group

$$
u_{d, \delta}: f(k) \rightarrow e^{3 \delta / 2} f\left(e^{\delta} k\right)
$$

of dilations on $L^{2}\left(\mathbb{R}^{n}\right)$. This group acts on creation and annihilation operators $a_{r}^{\#}(f)$ on the Fock space, $\mathcal{H}_{r}$, according to the rule

$$
\hat{U}_{d, \delta} a_{r}^{\#}(f) \hat{U}_{d, \delta}^{-1}=a_{r}^{\#}\left(u_{d, \delta} f\right), \quad \hat{U}_{d, \delta} \Omega_{r j}=\Omega_{r j} .
$$

We lift this group to the positive-temperature Hilbert space, (2.33), according to the formula

$$
U_{d, \delta}=\mathbf{1}_{p} \otimes \mathbf{1}_{p} \otimes \hat{U}_{d, \delta} \otimes \hat{U}_{d,-\delta} \otimes \hat{U}_{d, \delta} \otimes \hat{U}_{d,-\delta} .
$$

Note that we could dilate each reservoir by a different amount. However, this does not give us any advantage, so to keep notation simple we use the same dilation parameter for both reservoirs.

We record for future reference how the group $U_{d, \delta}$ acts on the Liouville operator $L_{0}$ and the positive-temperature photon number operator $N:=\sum_{j=1}^{2} N_{j}$, where

$$
N_{j}:=\int\left[a_{\ell, j}^{*}(k) a_{\ell, j}(k)+a_{r, j}^{*}(k) a_{r, j}(k)\right] d^{3} k,
$$

and the operators $a_{\{\ell, r\}, j}^{\#}(k)$ were introduced after (2.34). We have (below we do not display the identity operators):

$$
U_{d, \delta} L_{r j} U_{d, \delta}^{-1}=\cosh (\delta) L_{r j}+\sinh (\delta) \Lambda_{j},
$$

where $\Lambda_{j}$ is the positive operator on the $j$ th reservoir Hilbert space given by

$$
\Lambda_{j}=\int \omega(k)\left(a_{\ell, j}^{*}(k) a_{\ell, j}(k)+a_{r, j}^{*}(k) a_{r, j}(k)\right) d^{3} k,
$$


and

$$
U_{d, \delta} N_{j} U_{d, \delta}^{-1}=N_{j} .
$$

Now we define a one-parameter group of translations. It can be defined as one-parameter group arising from transformations of the underlying physical space similarly to the dilation group. This is done in Appendix B. We define here the translation group by means of the selfadjoint generator $T:=\sum_{j=1}^{2} T_{j}$, where

$$
T_{j}=\int\left[a_{\ell, j}^{*}(k) \gamma a_{\ell, j}(k)+a_{r, j}^{*}(k) \gamma a_{r, j}(k)\right] d^{3} k .
$$

The operator $\gamma=i(\hat{k} \cdot \nabla+\nabla \cdot \hat{k})$, with $\hat{k}=k /|k|$, is a symmetric, but not a self-adjoint operator. Nevertheless, the operators $T_{j}, j=1,2$, are self-adjoint [25]. Thus the operator $T$ is self-adjoint as well. We define the one-parameter group of translations as

$$
U_{t, \tau}:=\mathbf{1}_{p} \otimes \mathbf{1}_{p} \otimes e^{i \tau T} .
$$

Equations (6.7)-(6.8) imply the following expressions for the action of this group on the Liouville operators:

$$
U_{t, \tau} L_{r j} U_{t, \tau}^{-1}=L_{r j}+\tau N_{j} .
$$

Observe that neither the dilation nor the translation group affects the particle vectors, and that $U_{t, \tau} N_{j} U_{t, \tau}^{-1}=N_{j}$.

Now we want to apply the product of these transformations to the full operator $K=L_{0}+g I$. Since the dilation and translation transformations do not commute we have to choose the order in which we apply them. Since the operator $\Lambda=\sum_{j} \Lambda_{j}$ is not analytic under the translations while the operator $N$ is analytic under dilations we apply first the translation and then the dilation transformation. We define the combined translation-dilation transformation as

$$
U_{\theta}=U_{d, \delta} U_{t, \tau}
$$

where $\theta=(\delta, \tau)$. Note that $U_{\theta}$ leaves the finite-particle space $\mathcal{F}_{0}$, as well as $\operatorname{Dom}(\Lambda) \cap \operatorname{Dom}(N)$ invariant, for $\theta \in \mathbb{R}^{2}$.

In what follows we will use the notation $|\theta|=(|\delta|,|\tau|), \operatorname{Im} \theta=(\operatorname{Im} \delta, \operatorname{Im} \tau)$, and similarly for $\operatorname{Re} \theta$, and

$$
\operatorname{Im} \theta>0 \Longleftrightarrow \operatorname{Im} \delta>0 \wedge \operatorname{Im} \tau>0 .
$$

Now we are ready to define a complex deformation of the operator $K$. On the set $\operatorname{Dom}(\Lambda) \cap \operatorname{Dom}(N)$ we define for $\theta \in \mathbb{R}^{2}$

$$
K_{\theta}:=U_{\theta} K U_{\theta}^{-1} .
$$

Recalling the decomposition $K=L_{0}+g I$, where $L_{0}:=L_{p}+L_{r}, L_{r}:=\sum_{j=1}^{2} L_{r j}$ and $I=V-V_{-i / 2}^{\prime}$, we have

$$
K_{\theta}=L_{0, \theta}+g I_{\theta},
$$


where the families $L_{0, \theta}$ and $I_{\theta}$ are defined accordingly. Due to (6.4), (6.6) and (6.9) we have:

$$
L_{0, \theta}=L_{p}+\cosh (\delta) L_{r}+\sinh (\delta) \Lambda+\tau N,
$$

where $\theta=(\delta, \tau)$, and $\Lambda=\sum_{j=1}^{2} \Lambda_{j}$. An explicit expression for the family $I_{\theta}$ is given in Appendix B.2 (see (B.2.5) and (B.2.7)).

Similarly, we define the family $K_{(s) \theta}:=U_{\theta} K_{(s)} U_{\theta}^{-1}$ (recall (5.12)).

The operator families above are well defined for real $\theta$. Our task is to define them as analytic families on the strips

$$
S_{\theta_{0}}^{ \pm}=\left\{\theta \in \mathbb{C}^{2} \mid 0< \pm \operatorname{Im} \theta<\theta_{0}\right\}
$$

where $\theta_{0}=\left(\delta_{0}, \tau_{0}\right)>0$ is the same as in Condition (B). Recall that the inequality $\pm \operatorname{Im} \theta<\theta_{0}$ is equivalent to the following inequalities: $\pm \operatorname{Im} \delta<\delta_{0}$ and $\pm \operatorname{Im} \tau<\tau_{0}$. (The fact that analyticity in a neighbourhood of a fixed $\theta \in S_{\theta_{0}}^{ \pm}$implies analyticity in the corresponding strip in which $\operatorname{Re} \theta$ is not constraint follows from the explicit formulas (6.14), (B.2.5) and (B.2.7).) The analytic continuations (if they exist) are denoted by the same symbols.

We define the family $K_{\theta}$ for $\theta \in\left\{\theta \in \mathbb{C}^{2}|| \operatorname{Im} \theta \mid<\theta_{0}\right\}$ by the explicit expressions (6.13), (6.14), (B.2.5) and (B.2.7). Clearly, $\operatorname{Dom}(\Lambda) \cap \operatorname{Dom}(N) \subset$ $\operatorname{Dom}\left(L_{0 \theta}\right)$ and on this domain the family $L_{0 \theta}$ is manifestly strongly analytic in $\theta \in\left\{\theta \in \mathbb{C}^{2}|| \operatorname{Im} \theta \mid<\theta_{0}\right\}$. It is shown in Appendix B that for $|\operatorname{Im} \theta|<\theta_{0}$ we have $\operatorname{Dom}\left(\Lambda^{1 / 2}\right) \subset \operatorname{Dom}\left(I_{\theta}\right)$ and $I_{\theta} f$ is analytic $\forall f \in \operatorname{Dom}\left(\Lambda^{1 / 2}\right)$. Here Condition (B) of Section 3 is used. Hence the family $K_{\theta}$ for $\theta \in\left\{\theta \in \mathbb{C}^{2}|| \operatorname{Im} \theta \mid<\theta_{0}\right\}$ is bounded from $\operatorname{Dom}(\Lambda) \cap \operatorname{Dom}(N)$ to $\mathcal{H}$ (and $K_{\theta} f$ is analytic in $\theta \in\left\{\theta \in \mathbb{C}^{2}|| \operatorname{Im} \theta \mid<\theta_{0}\right\}$, $\forall f \in \operatorname{Dom}(\Lambda) \cap \operatorname{Dom}(N)$ ). Moreover, for $|\operatorname{Im} \theta|>0$ the operators $K_{\theta}$ are closable on the domain $\operatorname{Dom}(\Lambda) \cap \operatorname{Dom}(N)$, since their adjoints are defined on this dense domain. We denote their closures by the same symbols.

However, $\left\{K_{\theta} \mid \operatorname{Im} \theta<\theta_{0}\right\}$ is not an analytic family in the sense of Kato. The problem here is the lack of coercivity - the perturbation $I$ is not bounded relatively to the unperturbed operator $L_{0}$. To compensate for this we have chosen the deformation $U_{\theta}$ in such a way that the operator $M_{\theta}:=\operatorname{Im} L_{0, \theta}$ is coercive for $\operatorname{Im} \theta>0$, i.e., the perturbation $I_{\theta}$ as well as $\operatorname{Re} L_{0, \theta}$ are bounded relative to this operator. The problem here is that $M_{\theta} \rightarrow 0$ as $\operatorname{Im} \theta \rightarrow 0$ so we have to proceed carefully.

Everything said about $K_{\theta}$ applies also to the family $K_{(s) \theta}$.

The next result gives some analyticity properties and some global spectral properties of $K_{(s) \theta}$.

Theorem 6.1 ([25]). Assume that Condition (B) holds and let $\theta_{0}=\left(\delta_{0}, \tau_{0}\right)$ be as in that condition. Take an

$$
a>\frac{g^{2}}{\sin (\operatorname{Im} \delta)} C_{0}^{2}\left(\sum_{j=1}^{2}\left\|G_{j}\right\|_{\delta \beta_{j}, 1 / 2, \theta}\right)^{2},
$$


where $C_{0}:=C\left(1+\beta_{1}^{-1 / 2}+\beta_{2}^{-1 / 2}\right)$, and where $C$ is a constant depending only on $\tan \delta_{0}$. Then we have:

(i) $\{z \in \mathbb{C} \mid \operatorname{Im} z \leq-a\} \subseteq \rho\left(K_{(s) \theta}\right)$ (resolvent set) if either $s \in \mathbb{R}$ and $\theta \in \overline{S_{\theta_{0}}^{+}}$, or if $s \in S_{\epsilon}$ and $\theta \in S_{\theta_{0}}^{+}$;

(ii) Let $C_{a, b}$ be the truncated cone

$C_{a, b}:=\left\{z \in \mathbb{C}\left|\operatorname{Im} z>-\frac{a}{2},\right| \operatorname{Re} z \mid<2\left[(\sin b)^{-1}+a / 4\right](\operatorname{Im} z+a)+\left\|L_{p}\right\|+1\right\}$.

Take $s \in S_{\varepsilon}, \theta \in S_{\theta_{0}}^{+}$, and take $a$ as in (6.16). Then $\sigma\left(K_{\theta}\right) \subset C_{a, \operatorname{Im} \delta}$, and for $z \in \mathbb{C} \backslash C_{a, \operatorname{Im} \delta}$ we have

$$
\left\|\left(K_{\theta}-z\right)^{-1}\right\| \leq\left(\operatorname{dist}\left(z, C_{a, \operatorname{Im} \delta}\right)\right)^{-1} .
$$

(iii) The family $K_{(s) \theta}, s \in S_{\epsilon}, \theta \in \overline{S_{\theta_{0}}^{+}}$, is analytic of type $A$ (in the sense of Kato) in $\theta \in S_{\theta_{0}}^{+}$, for all $s \in S_{\epsilon}$, and in $s \in S_{\epsilon}$, for all $\theta \in S_{\theta_{0}}^{+}$;

(iv) Let $s \in \mathbb{R}$. For any $u$ and $v$ which are $U_{\theta}$-analytic in a strip $\left\{\theta \in \mathbb{C}^{2} \mid 0 \leq \operatorname{Im} \theta<\theta_{1}\right\}$, for some $\theta_{1}=\left(\delta_{1}, \tau_{0}\right), \delta_{1} \in\left[0, \min \left\{\pi / 3, \theta_{0}\right\}\right)$, the following relation holds:

$$
\left\langle u,\left(K_{(s)}-z\right)^{-1} v\right\rangle=\left\langle u_{\bar{\theta}},\left(K_{(s) \theta}-z\right)^{-1} v_{\theta}\right\rangle,
$$

where $u_{\theta}=U_{\theta} u$, etc., $\operatorname{Im} z \leq-a$ and $0<\operatorname{Im} \theta<\theta_{1} / 2$.

Proof. Statements (i), (iii) and (iv) are special cases of Theorem 5.1 in [25], with the exception of the assertion about analyticity of $s \mapsto K_{(s) \theta}$ in (iii). This assertion is easily proven by noticing that $\partial_{s}\left(K_{(s) \theta}-z\right)^{-1}=-\left(K_{(s) \theta}-z\right)^{-1}\left(\partial_{s} V_{s}^{\prime}\right)\left(K_{(s) \theta}-\right.$ $z)^{-1}$. Statement (ii) is the content of Proposition 5.2 in [25].

\section{Spectral analysis of $K_{\theta}$}

In what follows, given a self-adjoint operator $A$ and $a \in \mathbb{R}$ we use the notation $\chi_{A \leq a}$ for the spectral projection of $A$ associated to the set $\{\lambda \in \mathbb{R} \mid \lambda \leq a\}$ and similarly for $\chi_{A \geq a}$ and $\chi_{A=a}$, etc. Fix a $\theta$ satisfying $0<\operatorname{Im} \theta<\theta_{0}$.

Theorem 7.1. Assume Conditions (A)-(D). Let $\theta_{0}$ be the same as in Condition (B), let $g_{1}$ be as in Theorem 3.1 and let $\theta_{0}>\operatorname{Im} \theta=\left(\delta^{\prime}, \tau^{\prime}\right)>0$. If $0<|g|<g_{1}$ and $\tau^{\prime}>|g|^{2+\alpha}$ then

(a) 0 is an isolated and simple eigenvalue of $K_{\theta}$;

(b) $\sigma\left(K_{\theta}\right) \backslash\{0\} \subset\left\{z \in \mathbb{C}^{+} \mid \operatorname{Im} z \geq \min \left(c g^{2}, \frac{1}{2} \tau^{\prime}\right)\right\}$,

for some $c>0$, independent of $\theta$.

Theorem 7.1 is proven at the end of this section. Together with Theorem 6.1 it gives the following picture for the spectrum of $K_{\theta}$. 


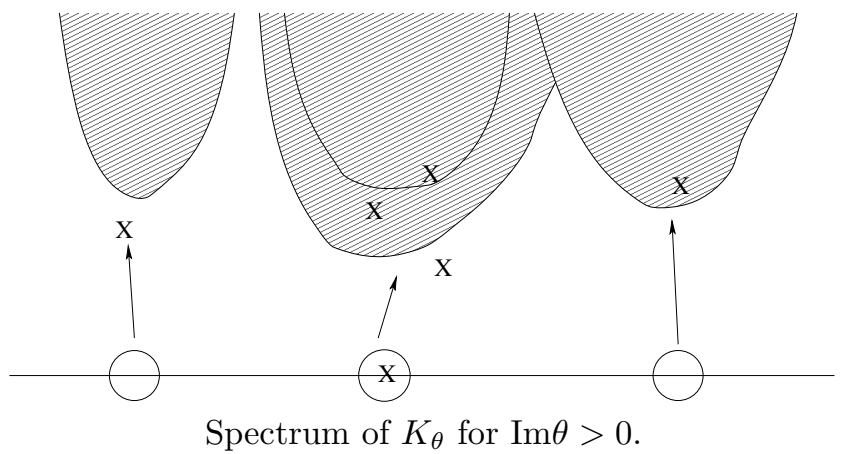

The motion of resonances bifurcating out of the eigenvalues of $L_{0}$ is governed, to second order in the coupling constant $g$, by level shift operators, see [24,25] for a discussion closest to the situation at hand. Let $e$ be an eigenvalue of $L_{p}$ and let $\Lambda_{e}$ be the level shift operator acting on $\operatorname{Ran} \chi_{L_{p}=e}$, defined by

$$
\Lambda_{e}:=-P_{e} I\left(L_{0}-e+i 0\right)^{-1} I P_{e},
$$

where $P_{e}=\chi_{L_{p}=e} \otimes \chi_{L_{r}=0}$. The notation $+i 0$ in (7.1) stands for the limit of $i \epsilon$ as $\epsilon \downarrow 0$. The following result summarizes properties of the level shift operators which are essential for the proof of Theorem 7.1 .

Theorem 7.2. Assume Condition (C). The level shift operators $\Lambda_{e},(7.1)$, satisfy

$$
\begin{aligned}
& \sigma\left(\Lambda_{e}\right) \subset \overline{\mathbb{C}^{+}} \\
& \sigma\left(\Lambda_{e}\right) \cap \mathbb{R}= \begin{cases}\emptyset & \text { if } e \neq 0 \\
\{0\} & \text { if } e=0\end{cases} \\
& \operatorname{dim} \operatorname{Ker}\left(\Lambda_{e=0}\right)=1 .
\end{aligned}
$$

Furthermore, there is a $\gamma_{0}>0$ which does not depend on the inverse temperatures, s.t. $\operatorname{Im} \Lambda_{e} \geq \gamma_{0}$, for all $e \neq 0$. Moreover, if Condition (D) is satisfied, then there is a $\delta_{0}>0$ which does not depend on the inverse temperatures, s.t. $\operatorname{Im}\left(\sigma\left(\Lambda_{0}\right) \backslash\{0\}\right) \geq \delta_{0}$.

We prove this theorem in Section 10.

Proof of Theorem 7.1. That 0 is an eigenvalue of $K_{\theta}$ follows readily from the equations $K \Omega=0$ and the fact that $\Omega$ is $U_{\theta}$-analytic in the strip (6.15), as we show in Lemma 7.3 below. So we have

$$
K_{\theta} \Omega_{\theta}=0
$$

where $\Omega_{\theta}:=U_{\theta} \Omega$.

Lemma 7.3. $\Omega$ is $U_{\theta}$-analytic, for $\theta=(\delta, \tau) \in S_{\theta_{0}}^{ \pm}$, see $(6.15)$.

Proof of Lemma 7.3. This follows from the Dyson series expansion (4.29) given in Lemma 4.2, and the analyticity condition (B). 
Let $\rho_{0} \in(0, \sigma / 2)$, where $\sigma$ is given in (3.9), and consider the half-space

$$
S=\left\{z \in \mathbb{C} \mid \operatorname{Im} z<\frac{1}{4} \rho_{0} \sin (\operatorname{Im} \delta) \rho_{0}\right\} .
$$

We decompose this region into the strips $S_{e}=\left\{z \in S|| \operatorname{Re} z-e \mid \leq \rho_{0}\right\}$, where $e \in$ $\sigma\left(L_{p}\right)$, and the complement. The following result is a special case of Theorem 6.1 of $[25]$.

Theorem 7.4. Assume that condition (B) holds and that there are constants $\gamma_{0}>0$ and $\delta_{0}>0$ satisfying $\operatorname{Im} \Lambda_{e} \geq \gamma_{0}$ for all $0 \neq e \in \sigma\left(L_{p}\right)$ and $\operatorname{Im}\left(\sigma\left(\Lambda_{0}\right) \backslash\{0\}\right) \geq \delta_{0}$. Take $0<|g|<\sqrt{\rho_{0}} g_{0}$ and let $\alpha=(\mu-1 / 2) /(\mu+1 / 2)$, where $\mu>1 / 2$ is given in Condition (B). Then

1. We have $\sigma\left(K_{\theta}\right) \cap S \subset \bigcup_{e \in \sigma\left(L_{p}\right)} S_{e}$.

2. Choose $\rho_{0}=|g|^{2-2 \alpha}$. There is a $C>0$ s.t. if $0<|g|<C\left(\gamma_{0}\right)^{1 / \alpha}$, then, for all $e \neq 0$,

$$
\sigma\left(K_{\theta}\right) \cap S_{e} \subset\left\{z \in \mathbb{C} \mid \operatorname{Im} z \geq \frac{1}{2} g^{2} \gamma_{0}\right\},
$$

3. Choose $\rho_{0}=|g|^{2-2 \alpha}$. There is a $C>0$ s.t. if $0<|g|<C \min \left[\left(\delta_{0}\right)^{1 / \alpha},\left(\tau^{\prime}\right)^{\frac{1}{2+\alpha}}\right]$ then

$$
\sigma\left(K_{\theta}\right) \cap S_{0} \subset\left\{z_{0}\right\} \cup\left\{z \in \mathbb{C} \mid \operatorname{Im} z \geq \frac{1}{2} \min \left(g^{2} \delta_{0}, \tau^{\prime}\right)\right\},
$$

where $z_{0}$ is a simple isolated eigenvalue of $K_{\theta}$, satisfying $\left|z_{0}\right|=O\left(|g|^{2+\alpha}\right)$.

For a coupling constant satisfying

$$
0<|g|<\min \left[\left(g_{0}\right)^{1 / \alpha},\left(\gamma_{0}\right)^{1 / \alpha},\left(\delta_{0}\right)^{1 / \alpha},\left(\tau^{\prime}\right)^{\frac{1}{2+\alpha}}\right]
$$

all three parts of Theorem 7.4 apply. Thus $K_{\theta}$ has a simple isolated eigenvalue $\left\{z_{0}\right\}$ in a neighbourhood $O\left(|g|^{2+\alpha}\right)$ of the origin, and $\sigma\left(K_{\theta}\right) \backslash\left\{z_{0}\right\} \subset\left\{z \in \mathbb{C}_{+} \mid \operatorname{Im} z \geq\right.$ $\left.\min \left(c g^{2}, \frac{1}{2} \tau^{\prime}\right)\right\}$, where $c=\frac{1}{2} \min \left(\gamma_{0}, \delta_{0}\right)$. In order to complete the proof of Theorem 7.1 we only need to remark that $z_{0}=0$ since zero is an eigenvalue of $K_{\theta}$, see (7.5). (One can also give a dynamical argument to prove that $z_{0}=0$, see the remark after (8.12).)

\section{Resolvent representation and pole approximation}

In order to study the long-time behaviour of the evolution $U(t)=e^{i K t}$ (defined on the domain $\pi(\mathcal{A}) \Omega$ by $(4.2))$ we relate it to an object which we understand relatively well, namely the resolvent $\left(K_{\theta}-z\right)^{-1}$ of the deformation $K_{\theta}$, defined in Section 6 . The main result of this section is 
Theorem 8.1. Assume that Conditions (A), (B) and (4.1) hold. Let $\phi$ and $\Psi$ be $U_{\theta}$-analytic vectors, and let $\Psi=\pi(A) \Omega$ for some $A \in \mathcal{A}$. We have the following representation

$$
\left\langle\phi, e^{i K t} \Psi\right\rangle=-\frac{1}{2 \pi i} \oint_{\Gamma}\left\langle\phi_{\bar{\theta}},\left(K_{\theta}-z\right)^{-1} \Psi_{\theta}\right\rangle e^{i z t} d z,
$$

where $\Psi_{\theta}=U_{\theta} \Psi$ (similarly for $\phi$ or any other vector), $\theta \in S_{\theta_{0}}^{+}$, and $\Gamma$ is the path

$$
\begin{aligned}
\Gamma:=\left\{z=\lambda-i \tau^{\prime} / 3,|\lambda| \leq C\right\} \cup\left\{z=\lambda-i 2 \tau^{\prime} / 3+i \lambda \tau^{\prime} / 3 C, \lambda \geq C\right\} \\
\cup\left\{z=\lambda-i 2 \tau^{\prime} / 3-i \lambda \tau^{\prime} / 3 C, \lambda \leq-C\right\}
\end{aligned}
$$

for a sufficiently large constant $C$. The integral on the r.h.s. of (8.1) is well defined in virtue of Theorem $6.1,(6.17)$, and the estimate $\left|e^{i z t}\right| \leq e^{-\lambda \tau^{\prime} / 3 C}$ on the infinite branches of $\Gamma$.

Proof. In the proof below, the vectors $\phi$ and $\Psi$ are as in the theorem. To prove the equality in (8.1) we use the family $K_{(s)}$ of operators defined in (5.12)-(5.13) (here we use Conditions (A) and (4.1)). Note that $\Omega \in \operatorname{Dom}\left(e^{\nu N}\right)$ for any $\nu>0$, as follows from the relation $\Omega_{0} \in \operatorname{Dom}\left(e^{\nu N} e^{-\beta L^{(\ell)} / 2}\right)$ which is shown in the same way as the relation $\Omega_{0} \in \operatorname{Dom}\left(e^{-\beta L^{(\ell)} / 2}\right)$, see Lemma 4.2 .

Next, we define the operator $e^{i K_{(s)} t}$ as follows: $e^{i K_{(s)} t} \Omega$ is given by a Dyson expansion, where the part $-g V_{s}^{\prime}$ of $K_{(s)}$ is treated as a perturbation. The fact that the Dyson series converges is easily seen from the relation $\Omega \in \operatorname{Dom}\left(e^{\nu N}\right), \nu>0$, shown above. Moreover, it is clear that this series defines a vector which is analytic in $s \in S_{\varepsilon}$, i.e., $s \mapsto e^{i K_{(s)} t} \Omega$ is analytic for $s \in S_{\varepsilon}$. We define the action of $e^{i K_{(s)} t}$ on vectors $\pi(A) \Omega, A \in \mathcal{A}$ (which form a dense set), by

$$
e^{i K_{(s)} t} \pi(A) \Omega=\sigma^{t}(\pi(A)) e^{i K_{(s)} t} \Omega .
$$

Consequently, the map $s \mapsto e^{i K_{(s)} t} \pi(A) \Omega$ is analytic for $s \in S_{\varepsilon}$. For $s=-i / 2$ this definition gives $e^{i K t}$.

Since $K_{(s)}$ is self-adjoint for $s$ real we derive from Stone's formula

$$
\left\langle\phi, e^{i K_{(s)} t} \Psi\right\rangle=-\frac{1}{2 \pi i} \oint_{\mathbb{R}-i}\left\langle\phi,\left(K_{(s)}-z\right)^{-1} \Psi\right\rangle e^{i z t} d z, \quad s \in \mathbb{R} .
$$

Next, using $e^{i z t}=\frac{1}{i t} \frac{\partial}{\partial(\operatorname{Re} z)} e^{i z t}$ and integrating by parts we can represent the r.h.s. of $(8.4)$ as

$$
R H S(8.4)=-\frac{1}{2 \pi i} \int_{\mathbb{R}-i}\left\langle\phi,\left(K_{(s)}-z\right)^{-2} \Psi\right\rangle e^{i z t} d z .
$$

Now we perform the spectral deformation, Theorem 6.1 , (iv), to obtain for $\theta_{1} / 2>$ $\operatorname{Im} \theta>0$ (where $\theta_{1}$ is given in Theorem 6.1 , (iv); here we use Conditions (B))

$$
R H S(8.4)=-\frac{1}{2 \pi i} \frac{1}{i t} \int_{\mathbb{R}-i}\left\langle\phi_{\bar{\theta}},\left(K_{(s) \theta}-z\right)^{-2} \Psi_{\theta}\right\rangle e^{i z t} d z .
$$


The integral converges since due to (6.17) we have $\left\|\left(K_{(s) \theta}-z\right)^{-n}\right\| \leq C_{n}\langle\operatorname{Re} z\rangle^{-n}$ for $z \in \mathbb{R}-i \varepsilon$, where $\langle x\rangle:=\sqrt{1+x^{2}}$.

In (8.5) we deform the contour of integration from $\mathbb{R}-i$ to $\Gamma$ which is fine due to $(6.17)$, and we integrate by parts in the opposite direction of above, to obtain

$$
\left\langle\phi, e^{i K_{(s)} t} \Psi\right\rangle=-\frac{1}{2 \pi i} \oint_{\Gamma}\left\langle\phi_{\bar{\theta}},\left(K_{(s) \theta}-z\right)^{-1} \Psi_{\theta}\right\rangle e^{i z t} d z .
$$

Both sides of this expression are well defined and analytic for $s \in S_{\varepsilon}$ (see Theorem $6.1,(6.17)$, and after equation (8.3)). Since they are equal for real $s$ they are equal for all $s$ in their domain of analyticity. Taking $s=-i / 2$ in this formula gives (8.1).

It is shown in Section 7 that the operator family $K_{\theta}$ has a simple isolated eigenvalue at 0 and the rest of its spectrum is located in a truncated cone in $\left\{z \in \mathbb{C}^{+} \mid \operatorname{Im} z>\frac{1}{3} \tau^{\prime}\right\}$, where $\tau^{\prime}=\operatorname{Im} \tau$. In the integral on the r.h.s. of formula (8.1) we deform the contour of integration to

$$
\begin{aligned}
\Gamma^{\prime}:=\left\{z=\lambda+\frac{1}{3} i \tau^{\prime},|\lambda| \leq C\right\} \cup\{z=\lambda+ & \left.i \lambda \tau^{\prime} / 3 C, \lambda \geq C\right\} \\
& \cup\left\{z=\lambda-i \lambda \tau^{\prime} / 3 C, \lambda \leq-C\right\}
\end{aligned}
$$

where $C$ is sufficiently large. Picking up the residue from the simple eigenvalue 0 of $K_{\theta}$ we derive from $(8.1)$

$$
\left\langle\phi, e^{i K t} \Psi\right\rangle=\left\langle\phi_{\bar{\theta}}, P_{\theta} \Psi_{\theta}\right\rangle+O\left(\left\|\phi_{\bar{\theta}}\right\|\left\|\Psi_{\theta}\right\| e^{-\tau^{\prime} t / 3}\right),
$$

where $P_{\theta}=\frac{-1}{2 \pi i} \oint\left(K_{\theta}-z\right)^{-1} d z$ is the eigenprojection of $K_{\theta}$ corresponding to the simple and isolated eigenvalue 0 and the remainder bound is coming from the term $\frac{1}{2 \pi} \oint_{\Gamma^{\prime}}\left\langle\phi_{\bar{\theta}},\left(K_{\theta}-z\right)^{-1} \Psi_{\theta}\right\rangle e^{i z t} d z$. The contour integral is over a small circle around the origin and the path $\Gamma^{\prime}$ is defined in (8.7). This is the only place where we use that 0 is an isolated eigenvalue of $K_{\theta}$ - the fact we show using complex translation in addition to complex dilation.

$P_{\theta}$ is a rank one projection which is analytic in $\theta \in S_{\theta_{0}}^{+}$. One proves using a standard argument that it satisfies $K_{\theta} P_{\theta}=P_{\theta} K_{\theta}=0$. Hence, $P_{\theta}$ can be written as

$$
P_{\theta}=\left|\Omega_{\theta}\right\rangle\left\langle\Omega_{\frac{*}{\theta}}\right|,
$$

where $\Omega_{\theta}$ and $\Omega_{\frac{*}{\theta}}$ are zero eigenvectors of $K_{\theta}$ and its adjoint operator

$$
\left(K_{\theta}\right)^{*}=\left(K^{*}\right)_{\bar{\theta}}=: K_{\bar{\theta}}^{*},
$$

i.e., we have

$$
K_{\theta} \Omega_{\theta}=0 \text { and } K_{\frac{\theta}{\theta}}^{*} \Omega_{\bar{\theta}}^{*}=0,
$$

with the normalization $\left\langle\Omega_{\frac{*}{\theta}}^{*}, \Omega_{\theta}\right\rangle=1$. Since $P_{\theta}$ and $\Omega_{\theta}$ are analytic in $\theta \in S_{\theta_{0}}^{+}$, then so is $\Omega_{\bar{\theta}}^{*}$ in the variable $\bar{\theta} \in S_{\theta_{0}}^{-}$. (The possibility of the normalization, $\left\langle\Omega_{\frac{*}{\theta}}, \Omega_{\theta}\right\rangle \neq 0$, follows also from results of Section 11. Analyticity of $\Omega_{\bar{\theta}}^{*}$ in $\bar{\theta} \in S_{\theta_{0}}^{-}$can also be 
shown directly by using the analyticity and spectral properties of $K_{\bar{\theta}}^{*}$, see Section 7). Equation (8.9) implies

$$
\left\langle\phi_{\bar{\theta}}, P_{\theta} \Psi_{\theta}\right\rangle=\langle\phi, \Omega\rangle\left\langle\Omega_{\bar{\theta}}^{*}, \Psi_{\theta}\right\rangle .
$$

The resonance vector $\Omega^{*}$ appearing in (2.32) and (4.13) is defined by $\left\langle\Omega^{*}, \varphi\right\rangle=$ $\left\langle\Omega_{\theta}^{*},\left(U_{-\theta}\right)^{*} \varphi\right\rangle$.

Remark. We present here another proof of the relation $z_{0}=0$, where $z_{0}$ is the simple isolated eigenvalue of $K_{\theta}$ given in Theorem 7.4. Starting with the information on the spectrum of $K_{\theta}$ given in Theorem 7.4 and proceeding with a contour deformation as above we find that $\left\langle\phi, e^{i K t} \psi\right\rangle=e^{i z_{0} t}\left\langle\phi_{\bar{\theta}}, P_{\theta} \psi_{\theta}\right\rangle+O\left(e^{\left(\left|\operatorname{Im} z_{0}\right|-\tau^{\prime} / 3\right) t}\right)$ instead of (8.8). Applying this formula to $\phi=\psi=\Omega$ and using (4.2) we obtain $\langle\Omega, \Omega\rangle=e^{i z_{0} t}\langle\Omega, \Omega\rangle+O\left(e^{\left(\left|\operatorname{Im} z_{0}\right|-\tau^{\prime} / 3\right) t}\right)$. Since $\left|z_{0}\right|=O\left(|g|^{2+\alpha}\right) \ll \tau^{\prime}$ the error term tends to zero as $t \rightarrow \infty$, so by taking $\lim _{T \rightarrow \infty} \int_{0}^{T} d t$ on both sides we see that $z_{0}$ must be zero.

\section{Proof of Theorem 3.1}

Let $\psi$ be any $\omega_{0}$-normal state on $\mathcal{A}$. For the following reasoning, we may assume without loss of generality that $\psi(A)=\left\langle\Omega_{\psi}, \pi(A) \Omega_{\psi}\right\rangle$, for some $\Omega_{\psi} \in \mathcal{H}$. Since $\Omega$ is cyclic for $\pi(\mathcal{A})^{\prime}, \psi$ can be approximated as follows. For any $\epsilon>0$ there is a $Q \in \pi(\mathcal{A})^{\prime}$ s.t., for all $A \in \mathcal{A}$,

$$
|\psi(A)-\langle Q \Omega, \pi(A) Q \Omega\rangle|<\epsilon\|A\| .
$$

Applying this to $\psi^{t}(A)=\left\langle\Omega_{\psi}, \sigma^{t}(\pi(A)) \Omega_{\psi}\right\rangle$ pulling $Q$ through $\sigma^{t}(\pi(A))$ and taking into account (2.30), $U(t)=e^{i t K}$ and $U(t) \Omega=0$, we obtain

$$
\left|\psi^{t}(A)-\left\langle Q^{*} Q \Omega, e^{i t K} \pi(A) \Omega\right\rangle\right|<\epsilon\|A\|,
$$

uniformly in $t \in \mathbb{R}$. In order to examine the long time behaviour of $\left\langle Q^{*} Q \Omega, e^{i t K} \pi(A) \Omega\right\rangle$, via (8.8), we first approximate the vector $Q^{*} Q \Omega \in \mathcal{H}$ by

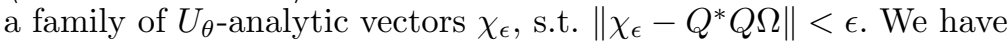

$$
\left|\left\langle Q^{*} Q \Omega-\chi_{\epsilon}, e^{i t K} \pi(A) \Omega\right\rangle\right|<\epsilon\left\|e^{i t K} \pi(A) \Omega\right\|=\epsilon\left\|\sigma^{t}(\pi(A)) \Omega\right\|<\epsilon\|A\| .
$$

It follows from (9.2), (9.3), (8.8) and (8.12) that

$$
\left|\psi^{t}(A)-\left\langle\chi_{\epsilon}, \Omega\right\rangle\left\langle\Omega_{\bar{\theta}}^{*},(\pi(A) \Omega)_{\theta}\right\rangle\right| \leq 2 \epsilon\|A\|+C\left\|\chi_{\epsilon, \bar{\theta}}\right\|\left\|(\pi(A) \Omega)_{\theta}\right\| e^{-\tau^{\prime} t / 3} .
$$

Since $\left\langle Q^{*} Q \Omega, \Omega\right\rangle \rightarrow 1$ as $\epsilon \rightarrow 0$, we have $\left\langle\chi_{\epsilon}, \Omega\right\rangle=1+o\left(\epsilon^{0}\right)$, where $o\left(\epsilon^{0}\right)$ denotes a quantity that vanishes in the limit $\epsilon \rightarrow 0$. Thus

$$
\begin{aligned}
\mid \psi^{t}(A)-\left\langle\chi_{\epsilon}, \Omega\right\rangle\left\langle\Omega_{\bar{\theta}}^{*},\right. & \left.(\pi(A) \Omega)_{\theta}\right\rangle \mid \\
& \geq\left|\psi^{t}(A)-\left\langle\Omega_{\frac{*}{\theta}}^{*},(\pi(A) \Omega)_{\theta}\right\rangle\right|-o\left(\epsilon^{0}\right)\left|\left\langle\Omega_{\frac{*}{\theta}},(\pi(A) \Omega)_{\theta}\right\rangle\right| .
\end{aligned}
$$


Combining this estimate with (9.4) we arrive at

$$
\begin{aligned}
\mid \psi^{t}(A) & -\left\langle\Omega_{\bar{\theta}}^{*},(\pi(A) \Omega)_{\theta}\right\rangle \mid \\
\leq & o\left(\epsilon^{0}\right)\left(\|A\|+\left|\left\langle\Omega_{\bar{\theta}}^{*},(\pi(A) \Omega)_{\theta}\right\rangle\right|\right)+C\left\|\chi_{\epsilon, \bar{\theta}}\right\|\left\|(\pi(A) \Omega)_{\theta}\right\| e^{-\tau^{\prime} t / 3},
\end{aligned}
$$

where $o\left(\epsilon^{0}\right)$ is independent of $A$ and $\theta$. In particular, taking first $t \rightarrow \infty$ and then $\epsilon \rightarrow 0$ yields

$$
\lim _{t \rightarrow \infty} \psi^{t}(A)=\left\langle\Omega_{\frac{*}{\theta}}^{*}(\pi(A) \Omega)_{\theta}\right\rangle .
$$

Equation (9.6) shows that $\left|\left\langle\Omega_{\bar{\theta}}^{*},(\pi(A) \Omega)_{\theta}\right\rangle\right| \leq\|A\|$. We re-inject this inequality into r.h.s. of (9.5) to arrive at

$$
\lim _{t \rightarrow \infty} \sup _{A \in \widehat{\mathcal{A}}} \frac{\left|\psi^{t}(A)-\eta(A)\right|}{\|\| A \| \mid}=0,
$$

where

$$
\widehat{\mathcal{A}}=\left\{A \in \mathcal{A} \mid \pi(A) \Omega \text { is } U_{\theta} \text {-analytic for }|\theta|<\theta_{0}\right\},
$$

and where ||$|\cdot|||$ is the norm on $\widehat{\mathcal{A}}$ defined by

$$
\|A\|\|=\| A\left\|+\sup _{|\theta|<\theta_{0}}\right\|(\pi(A) \Omega)_{\theta} \|, \quad \text { for } \quad A \in \widehat{\mathcal{A}} .
$$

The state $\eta$ on $\widehat{\mathcal{A}}$ in (9.7) is given by

$$
\eta(A):=\left\langle\Omega_{\frac{*}{\theta}},(\pi(A) \Omega)_{\theta}\right\rangle
$$

it is independent of the deformation parameter $\theta$, if $0<\operatorname{Im} \theta<\theta_{0}$, and $0<g<g_{0}$ ( $g_{0}$ depends on $\sin (\operatorname{Im} \delta)$, see the equation after $\left.(3.9)\right)$.

Observe that $\mathbf{1} \in \widehat{\mathcal{A}}$, and that the normalization $\left\langle\Omega_{\frac{*}{\theta}}, \Omega_{\theta}\right\rangle=1$ implies $\eta(\mathbf{1})=1$. $\widehat{\mathcal{A}}$ is a linear subspace of $\mathcal{A}$, but not an algebra.

We show in Appendix C, Proposition C.1, that $\mathcal{A}_{1}$ (defined in (2.17)) is strongly dense in $\mathcal{A}$ (defined in (2.7)), and that any $A \in \mathcal{A}_{1}$ has the property that $\pi(A) \Omega$ is $U_{\theta}$-analytic, for $\theta$ in a neighbourhood of $\theta=0$. Hence $\mathcal{A}_{1} \subseteq \widehat{\mathcal{A}} \subseteq \mathcal{A}$, and therefore $\widehat{\mathcal{A}}$ is strongly dense in $\mathcal{A}$.

We have thus shown that for any $\omega_{0}$-normal state $\psi, \psi^{t} \rightarrow \eta$ as $t \rightarrow \infty$, where the convergence is understood in the $\||\cdot|||$-topology of linear functionals on $\widehat{\mathcal{A}}$.

It is clear from (9.7) that $|\eta(A)| \leq\|A\|$, for $A \in \widehat{\mathcal{A}}$, hence $\eta$ extends to a bounded positive linear functional on the Banach space of observables

$$
\mathcal{A}_{0}:=\|\cdot\| \text {-closure of } \widehat{\mathcal{A}},
$$

normalized as $\eta(\mathbf{1})=1$. Standard perturbation theory shows that $\eta(A), A \in \widehat{\mathcal{A}}$, is analytic in the coupling constant $g$.

Observe that we can rewrite the state $\eta(A)$ also in the form

$$
\eta(A)=\operatorname{Tr}\left(\pi(A)_{\theta} P_{\theta}\right)
$$


where $\pi(A)_{\theta}:=U_{\theta} \pi(A) U_{\theta}^{-1}$. Formally one can undo the rotation in (9.10) to obtain (4.14) with $\Omega^{*}:=U_{\bar{\theta}}^{-1} \Omega_{\bar{\theta}}^{*}$. However, in the non-equilibrium situation $\Omega^{*} \notin$ $\mathcal{H}$ ! The set $\mathcal{A}_{1}$ is exactly the set on which (4.14) makes sense. Thus we gave a rigorous meaning to (4.14) and the NESS vector $\Omega^{*}$.

\section{Proof of Theorem 7.2}

Our task is to show that the spectrum of (7.1) lies in the upper complex half plane $\{\operatorname{Im} z>0\}$ if $e \neq 0$; and that it has a simple eigenvalue at zero and all the other eigenvalues lie in the upper complex half plane if $e=0$. While this analysis is standard in the case when $I$ is a selfadjoint operator (then the imaginary part of (7.1) is just $P_{e} I \delta\left(L_{0}-e\right) I P_{e}$, manifestly a non-negative operator; see, e.g., [8]), it needs some more thought in our case, where the interaction is non-selfadjoint. Let

$$
V_{j}=\pi\left(v_{j}\right), \quad \text { and } \quad V_{j}^{\prime}=\pi^{\prime}\left(v_{j}\right) .
$$

The main ingredient of the proof is

Proposition 10.1. Assume Conditions (A). We have

$$
\begin{array}{r}
\Lambda_{e}=\left(e^{-\beta_{p} H_{p} / 2} \otimes \mathbb{1}_{p}\right)\left[\sum_{j=1,2}\left(e^{\beta_{j} H_{p} / 2} \otimes \mathbb{1}_{p}\right) \Lambda_{j e}\left(e^{-\beta_{j} H_{p} / 2} \otimes \mathbb{1}_{p}\right)\right] \\
\times\left(e^{\beta_{p} H_{p} / 2} \otimes \mathbb{1}_{p}\right), \\
\text { where, setting } \mathcal{R}:=\left(L_{0}-e+i 0\right)^{-1}, \\
\Lambda_{j e}=-P_{e}\left(V_{j}-V_{j}^{\prime}\right) \mathcal{R}\left(V_{j}-V_{j}^{\prime}\right) P_{e} .
\end{array}
$$

Notice that (10.1) shows that the spectrum of $\Lambda_{e}$ is independent of $\beta_{p}$.

The importance of (10.1) is that it relates $\Lambda_{e}$ to the operators $\Lambda_{j e}$ whose spectral characteristics are well known. Indeed, $\Lambda_{j e}$ are the level shift operators corresponding to the reservoir $j$ coupled to the particle system, studied in $[8,24]$.

Before proceeding to the proof we examine some consequences of this proposition. We assume Conditions (C) and (D) in addition to Conditions (A).

The case $e \neq 0$. Let us assume that the nonzero eigenvalues of $L_{p}$ are simple, i.e., $E_{i}-E_{j}=E_{m}-E_{n} \Leftrightarrow i=m, j=n$. For a treatment of the more general case where $E_{i}-E_{j}=E_{m}-E_{n}$, for $(i, j) \neq(m, n)$, with simple $E_{j}$, we refer to [26]. Since $P_{e}$ is of rank one $\Lambda_{e}$ is just a complex number, namely the sum of $\Lambda_{1 e}+\Lambda_{2 e}$ (the dependence on $\beta_{1}, \beta_{2}$ disappears). Under condition (3.5), one has $\operatorname{Im} \Lambda_{e} \geq \gamma_{0}$, where $\gamma_{0}$ is a strictly positive constant which is independent of the inverse temperatures, see [8]). This shows (7.2) and (7.3) for $e \neq 0$.

The case $e=0$. Zero is necessarily a degenerate eigenvalue of $L_{0}$, so the above reasoning does not apply. In particular, $\Lambda_{10}$ and $\Lambda_{20}$ do not commute. It is shown in $[24,26]$ that

$$
\Lambda_{j 0}=i \operatorname{Im} \Lambda_{j 0}=: i \Gamma_{j 0},
$$


where $\operatorname{Im} \Lambda_{j 0}:=\frac{1}{2 i}\left(\Lambda_{j 0}-\Lambda_{j 0}^{*}\right)$. We use here implicitly Condition (C) on the nondegeneracy of $H_{p}$; if the small system has degenerate energy levels then $\Lambda_{j 0}$ are not purely imaginary [24]. One shows as in $[8,24,26]$ that $\Gamma_{j 0}$ are real matrices having strictly negative off-diagonal entries, $\left(\Gamma_{j 0}\right)_{m n}<0$, for $m \neq n$, and satisfying

$$
\Gamma_{j 0} \Omega_{p}^{\left(\beta_{j}\right)}=0,
$$

where $\Omega_{p}^{\left(\beta_{j}\right)}$ is the particle Gibbs state at temperature $\beta_{j}$. Hence, since

$$
\left(e^{\left(\beta_{p}-\beta_{j}\right) H_{p} / 2} \otimes \mathbb{1}_{p}\right) \Omega_{p}^{\left(\beta_{p}\right)}=\sqrt{\frac{\operatorname{tr} e^{-\beta_{j} H_{p} / 2}}{\operatorname{tr} e^{-\beta_{p} H_{p} / 2}}} \Omega_{p}^{\left(\beta_{j}\right)},
$$

we see that

$$
\Gamma_{0} \Omega_{p}^{\left(\beta_{p}\right)}=\sum_{j=1,2}\left(e^{\left(-\beta_{p}+\beta_{j}\right) H_{p} / 2} \otimes \mathbb{1}_{p}\right) \sqrt{\frac{\operatorname{tr} e^{-\beta_{j} H_{p} / 2}}{\operatorname{tr} e^{-\beta_{p} H_{p} / 2}}} \Gamma_{j 0} \Omega_{p}^{\left(\beta_{j}\right)}=0,
$$

where $\Gamma_{0}:=-i \Lambda_{0}$. Thus, $\Omega_{p}^{\left(\beta_{p}\right)}$ is an eigenvector of the real matrix $\Gamma_{0}$ with eigenvalue zero. Notice that the vector $\Omega_{p}^{\left(\beta_{p}\right)}$ has strictly positive components, $\left[\operatorname{tr} e^{-\beta_{p} H_{p} / 2}\right]^{-1} e^{-\beta_{p} E_{n} / 2}$, in the orthonormal basis $\left\{\varphi_{n} \otimes \varphi_{n}\right\}$ of $\operatorname{Ran} \chi_{L_{p}=0}$ (where $\left.H_{p} \varphi_{n}=E_{n} \varphi_{n},\left\|\varphi_{n}\right\|=1\right)$. Moreover, the off-diagonal elements of the real matrix (which is not symmetric for $\beta_{1} \neq \beta_{2}$ ) $\Gamma_{0}$ are given by

$$
\begin{aligned}
\left(\Gamma_{0}\right)_{m, n} & =\sum_{j=1,2}\left[\left(e^{\left(-\beta_{p}+\beta_{j}\right) H_{p} / 2} \otimes \mathbb{1}_{p}\right) \Gamma_{j 0}\left(e^{-\left(-\beta_{p}+\beta_{j}\right) H_{p} / 2} \otimes \mathbb{1}_{p}\right)\right]_{m n} \\
& =-\pi \sum_{j=1,2} \frac{E_{m n}^{2} e^{-\left(\beta_{p}-\beta_{j}\right) E_{m n} / 2}}{\left|\sinh \left(\beta_{j} E_{m n} / 2\right)\right|} \int_{S^{2}} d \sigma\left|\left[G_{j}\left(E_{m n}, \sigma\right)\right]_{n m}\right|^{2}
\end{aligned}
$$

for $m>n$ (and similarly for $m<n$, see also [8,26]). Hence Condition (D) implies that $\left(\Gamma_{0}\right)_{m, n}<0$. A standard Perron-Frobenius argument shows that zero is a simple eigenvalue of $\Gamma_{0}$, and that $\sigma\left(\Gamma_{0}\right) \backslash\{0\} \subset \mathbb{C}^{+}$. This shows equations (7.3)(7.4) for $e=0$. It is shown in [8] that the gap at the bottom of the spectrum of $\Gamma_{j 0}, j=1,2$ has a lower bound which is independent of the inverse temperatures.

We now prove the existence of $\delta_{0}$, assuming that Condition (E) is satisfied. If $\operatorname{dim} \mathcal{H}_{p}=2$ then one eigenvalue of $\Lambda_{0}$ is zero and the other equals the trace of $\Lambda_{0}$. Expressions (10.1) and (10.3) show that $\operatorname{Tr}\left(\Lambda_{0}\right)=i\left[\operatorname{Tr}\left(\Gamma_{10}\right)+\operatorname{Tr}\left(\Gamma_{20}\right)\right]$. Thus the spectral gap of $\Lambda_{0}$ is the sum of the gaps of $\Gamma_{01}$ and $\Gamma_{02}$, which have lower bounds uniform in the inverse temperatures.

Next take $\operatorname{dim} \mathcal{H}_{p} \geq 3$. For $\delta \beta=\left|\beta_{1}-\beta_{2}\right|=0$ the matrix $\Lambda_{0}$ has the same spectrum as $\sum_{j} \Lambda_{j 0}$, see (10.1). An application of the minimax principle demonstrates that the spectral gap of the latter operator has to be at least as large as the maximum of the gaps of $\Gamma_{j 0}, j=1,2$. For small values of $\delta \beta$ (c.f. (3.6)), the existence of $\delta_{0}$ follows by perturbation theory.

Finally we consider the case where $\delta \beta$ and $\beta_{1}, \beta_{2}$ are large (see (3.6)). Let us take $\beta_{2}=\beta_{1}+\delta \beta$. As is easily seen from (10.1) we have

$$
\sigma\left(\Lambda_{0}\right)=i \sigma\left(\left[\Gamma_{10}+\left(e^{\delta \beta H_{p} / 2} \otimes \mathbb{1}\right) \Gamma_{20}\left(e^{-\delta \beta H_{p} / 2} \otimes \mathbb{1}\right)\right]\right) .
$$


Using the explicit expression for the matrix elements of $\Gamma_{20}$ in the basis $\varphi_{j} \otimes \varphi_{j}$ (which can be read off of relation (10.5) for off-diagonal terms, and is easy to obtain for the diagonal ones $)$, one verifies that the matrix $\left(e^{\delta \beta H_{p} / 2} \otimes \mathbb{1}\right) \Gamma_{20}\left(e^{-\delta \beta H_{p} / 2} \otimes \mathbb{1}\right)$ converges to a lower-triangular matrix $Q\left(\beta_{1}\right)$, in the limit $\delta \beta \rightarrow \infty$ (uniformly in $\beta_{1}$ ), and furthermore, that $Q\left(\beta_{1}\right) \rightarrow D$ as $\beta_{1} \rightarrow \infty$, where $D$ is a diagonal matrix with non-negative entries. The minimax principle implies that all but one eigenvalues of $\Gamma_{10}+D$ are greater than, or equal to the gap of $\Gamma_{10}$. From perturbation theory we know that for $\delta \beta$ and $\beta_{1}$ sufficiently large (independently of each other $)$, all but one eigenvalues of the operator $\Gamma_{10}+\left(e^{\delta \beta H_{p} / 2} \otimes \mathbb{1}\right) \Gamma_{20}\left(e^{-\delta \beta H_{p} / 2} \otimes \mathbb{1}\right)$ must have real part greater than, or equal to half of the gap of $\Gamma_{10}$. The existence of $\delta_{0}$ now follows from (10.6).

Proof of Proposition 10.1. Let $V=\sum_{j=1}^{2} V_{j}$ and $V^{\prime}=\sum_{j=1}^{2} V_{j}^{\prime}$. By the definition of the operator $I, I:=V-V_{-i / 2}^{\prime}$, and the relation $V_{-i / 2}^{\prime}=e^{\tilde{L} / 2} V^{\prime} e^{-\tilde{L} / 2}$ we have

$$
I=V-e^{\tilde{L} / 2} V^{\prime} e^{-\tilde{L} / 2}
$$

where $\tilde{L}:=\delta \beta_{p} L_{p}+\delta \beta_{1} L_{r 1}+\delta \beta_{2} L_{r 2}$ with $\delta \beta_{p}=\beta_{p}-\beta$ and $\delta \beta_{j}=\beta_{j}-\beta$. Using that $P_{e} e^{-\delta \beta_{p} L_{p} / 2}=P_{e} e^{-\delta \beta_{p} e / 2}$ we decompose

$$
\begin{aligned}
\Lambda_{e}= & P_{e} V \mathcal{R} V P_{e}+P_{e} V^{\prime} \mathcal{R} V^{\prime} P_{e} \\
& -P_{e} V \mathcal{R} e^{\tilde{L} / 2} V^{\prime} P_{e} e^{-\delta \beta_{p} e / 2}-P_{e} V^{\prime} e^{-\tilde{L} / 2} \mathcal{R} V P_{e} e^{\delta \beta_{p} e / 2} .
\end{aligned}
$$

Notice that $V$ and $\mathcal{R}$ commute with $\mathbb{1}_{p} \otimes e^{\delta \beta_{p} H_{p} / 2}$. Using this and the relation

$$
\left(\mathbf{1}_{p} \otimes e^{\delta \beta_{p} H_{p} / 2}\right) P_{e}=e^{-\delta \beta_{p} e / 2}\left(e^{\delta \beta_{p} H_{p} / 2} \otimes \mathbf{1}_{p}\right) P_{e},
$$

we obtain

$$
\begin{aligned}
P_{e} V \mathcal{R} V P_{e} & =e^{\delta \beta_{p} e / 2} P_{e}\left(e^{-\delta \beta_{p} H_{p} / 2} \otimes e^{\delta \beta_{p} H_{p} / 2}\right) V \mathcal{R} V P_{e} \\
& =e^{\delta \beta_{p} e / 2} P_{e}\left(e^{-\delta \beta_{p} H_{p} / 2} \otimes \mathbb{1}_{p}\right) V \mathcal{R} V\left(\mathbb{1}_{p} \otimes e^{\delta \beta_{p} H_{p} / 2}\right) P_{e} \\
& =\left(e^{-\delta \beta_{p} H_{p} / 2} \otimes \mathbb{1}_{p}\right) P_{e} V \mathcal{R} V P_{e}\left(e^{\delta \beta_{p} H_{p} / 2} \otimes \mathbb{1}_{p}\right) .
\end{aligned}
$$

Next, using (10.8) again, we find

$$
\begin{aligned}
P_{e} V \mathcal{R} & e^{\tilde{L} / 2} V^{\prime} P_{e} e^{-\delta \beta_{p} e / 2} \\
& =P_{e} V \mathcal{R}\left(e^{\delta \beta_{p} H_{p} / 2} \otimes e^{-\delta \beta_{p} H_{p} / 2}\right) e^{\left(\delta \beta_{1} L_{r 1}+\delta \beta_{2} L_{r 2}\right) / 2} V^{\prime} P_{e} e^{-\delta \beta_{p} e / 2} \\
& =P_{e}\left(\mathbb{1}_{p} \otimes e^{-\delta \beta_{p} H_{p} / 2}\right) V \mathcal{R} e^{\left(\delta \beta_{1} L_{r 1}+\delta \beta_{2} L_{r 2}\right) / 2} V^{\prime}\left(e^{\delta \beta_{p} H_{p} / 2} \otimes \mathbb{1}_{p}\right) P_{e} e^{-\delta \beta_{p} e / 2} \\
& =\left(e^{-\delta \beta_{p} H_{p} / 2} \otimes \mathbb{1}_{p}\right) P_{e} V \mathcal{R} e^{\left(\delta \beta_{1} L_{r 1}+\delta \beta_{2} L_{r 2}\right) / 2} V^{\prime} P_{e}\left(e^{\delta \beta_{p} H_{p} / 2} \otimes \mathbb{1}_{p}\right) .
\end{aligned}
$$


Treating the other two terms in (10.7) in a similar way, we arrive at

$$
\begin{aligned}
-\Lambda_{e}= & \left(e^{-\delta \beta_{p} H_{p} / 2} \otimes \mathbb{1}_{p}\right) P_{e}\left[V \mathcal{R} V+V^{\prime} \mathcal{R} V^{\prime}\right. \\
& -V \mathcal{R} e^{\left(\delta \beta_{1} L_{r 1}+\delta \beta_{2} L_{r 2}\right) / 2} V^{\prime} \\
& \left.-V^{\prime} \mathcal{R} e^{\left(-\delta \beta_{1} L_{r 1}-\delta \beta_{2} L_{r 2}\right) / 2} V\right] P_{e}\left(e^{\delta \beta_{p} H_{p} / 2} \otimes \mathbb{1}_{p}\right) .
\end{aligned}
$$

We now examine the term in [ $\cdots]$. Write $V=V_{1}+V_{2}, V^{\prime}=V_{1}^{\prime}+V_{2}^{\prime}$, where, recall, $V_{j}=\pi\left(v_{j}\right), V_{j}^{\prime}=\pi^{\prime}\left(v_{j}\right)$. Notice that we have $\left(\mathbb{1}_{p} \otimes \mathbb{1}_{p} \otimes P_{\Omega} \otimes \mathbb{1}_{2}\right) V_{1}\left(\mathbb{1}_{p} \otimes \mathbb{1}_{p} \otimes P_{\Omega} \otimes\right.$ $\left.\mathbb{1}_{2}\right)=0$, and similarly for $V_{2}$, from which it follows that the expression $P_{e}[\cdots] P_{e}$ in (10.9) splits into a sum

$$
\begin{aligned}
P_{e} \sum_{j=1,2}\left[V_{j} \mathcal{R} V_{j}+V^{\prime} \mathcal{R} V_{j}^{\prime}-V_{j} \mathcal{R} e^{\left(\delta \beta_{1} L_{r 1}+\delta \beta_{2} L_{r 2}\right) / 2} V_{j}^{\prime}\right. & \\
& \left.-V_{j}^{\prime} \mathcal{R} e^{\left(-\delta \beta_{1} L_{r 1}-\delta \beta_{2} L_{r 2}\right) / 2} V_{j}\right] P_{e} .
\end{aligned}
$$

We consider the $j=1$ term. Using that $L_{r 2}$ commutes with $V_{1}, V_{1}^{\prime}$ and that $L_{r 2} P_{e}=0$, we see that

$$
\begin{array}{rl}
P_{e} V_{1} & \mathcal{R} e^{\delta \beta_{1} L_{r 1}+\delta \beta_{2} L_{r 2}} V_{1}^{\prime} P_{e} \\
& =P_{e} V_{1} \mathcal{R} e^{\delta \beta_{1} L_{r 1} / 2} V_{1}^{\prime} P_{e}=P_{e} V_{1} \mathcal{R} e^{\delta \beta_{1} L_{0} / 2} e^{-\delta \beta_{1} L_{p} / 2} V_{1}^{\prime} P_{e} \\
& =P_{e}\left(\mathbb{1}_{p} \otimes e^{\delta \beta_{1} H_{p} / 2}\right) V_{1} \mathcal{R} e^{\delta \beta_{1} L_{0} / 2} V_{1}^{\prime}\left(e^{-\delta \beta_{p} H_{p} / 2} \otimes \mathbb{1}_{p}\right) P_{e} \\
& =\left(e^{\delta \beta_{1} H_{p} / 2} \otimes \mathbb{1}_{p}\right) P_{e} V_{1} \mathcal{R} e^{\delta \beta_{1}\left(L_{0}-e\right) / 2} V_{1}^{\prime} P_{e}\left(e^{-\delta \beta_{1} H_{p} / 2} \otimes \mathbb{1}_{p}\right) .
\end{array}
$$

All other terms in (10.9) for $j=1$, as well as the terms for $j=2$, are treated similarly and one arrives at

$$
\begin{aligned}
-\Lambda_{e}= & \left(e^{-\beta_{p} H_{p} / 2} \otimes \mathbb{1}_{p}\right) \sum_{j=1,2}\left(e^{\beta_{j} H_{p} / 2} \otimes \mathbb{1}\right) P_{e}\left[V_{j} \mathcal{R} V_{j}+V_{j}^{\prime} \mathcal{R} V_{j}^{\prime}\right. \\
& -V_{j} \mathcal{R} e^{\delta \beta_{j}\left(L_{0}-e\right) / 2} V_{j}^{\prime} \\
& \left.-V_{j}^{\prime} \mathcal{R} e^{-\delta \beta_{j}\left(L_{0}-e\right) / 2} V_{j}\right] P_{e}\left(e^{-\beta_{j} H_{p} / 2} \otimes \mathbb{1}_{p}\right)\left(e^{\beta_{p} H_{p} / 2} \otimes \mathbb{1}_{p}\right),
\end{aligned}
$$

where we used $\delta \beta_{p}-\delta \beta_{j}=\beta_{p}-\beta_{j}$. Hence, $-\Lambda_{e}=R H S(10.1)+\left(e^{-\delta_{p} H_{p}} \otimes\right.$ $\left.\mathbb{1}_{p}\right) R\left(e^{\delta_{p} H_{p}} \otimes \mathbb{1}_{p}\right)$, where

$$
\begin{aligned}
R= & \sum_{j=1,2}\left(e^{\beta_{j} H_{p} / 2} \otimes \mathbb{1}\right) P_{e}\left[V_{j} \mathcal{R}\left(1-e^{\delta \beta_{j}\left(L_{0}-e\right) / 2}\right) V_{j}^{\prime}\right. \\
& \left.+V_{j}^{\prime} \mathcal{R}\left(1-e^{-\delta \beta_{j}\left(L_{0}-e\right) / 2}\right) V_{j}\right] P_{e}\left(e^{-\beta_{j} H_{p} / 2} \otimes \mathbb{1}_{p}\right) .
\end{aligned}
$$

Since $L_{0}$ implements the free dynamics, we have that $e^{i z L_{0}} V_{j} e^{-i z L_{0}}$ commutes with $V_{j}^{\prime}$, for $z \in \mathbb{C}$. Using this, writing $\frac{1-e^{\delta \beta_{j}\left(L_{0}-e\right) / 2}}{L_{0}-e \pm i 0}=-\int_{0}^{\delta \beta_{j} / 2} d s e^{s\left(L_{0}-e\right)}$ and 
using that $P_{e} L_{0}=e P_{e}$, we see that

$$
P_{e} V_{j} \frac{\mathbf{1}-e^{\delta \beta_{j}\left(L_{0}-e\right) / 2}}{L_{0}-e-i 0} V_{j}^{\prime} P_{e}=-P_{e} V_{j}^{\prime} \frac{\mathbf{1}-e^{-\delta \beta_{j}\left(L_{0}-e\right) / 2}}{L_{0}-e+i 0} V_{j} P_{e} .
$$

Consequently, $R=0$. This concludes the proof of Proposition 10.1.

\section{Perturbation theory for NESS}

In this section we develop a perturbation theory for the NESS $\eta(A):=$ $\left\langle\Omega_{\bar{\theta}}^{*}, \pi(A)_{\theta} \Omega_{\theta}\right\rangle$. The vectors $\Omega_{\frac{*}{\theta}}^{*}$ and $\Omega_{\theta}$ are the zero eigenvectors of the operators $K_{\frac{\theta}{*}}^{*}$ and $K_{\theta}$ respectively, see (8.10) and (8.11). We derive perturbation expansions for $\Omega_{\frac{*}{\theta}}$ and $\Omega_{\theta}$, see (11.11) and (11.15) below, using the Feshbach maps introduced in $[6,7]$, and extended in $[5]$. We review the definitions and some properties of these maps referring the reader to $[5,7]$ for more detail. For simplicity we present here the original version, [6,7], though the refined one, [5], the smooth Feshbach map, is easier to use from a technical point of view.

Let $X$ be a Banach space and let $P$ be a projection on $X$. Define $\bar{P}:=\mathbf{1}-P$ and let $H_{\bar{P}}:=\bar{P} H \bar{P}$ and $R_{\bar{P}}(H):=\bar{P} H_{\bar{P}}^{-1} \bar{P}$ if $H_{\bar{P}}$ is invertible on $\operatorname{Ran} \bar{P}$. We define the Feshbach map $F_{P}$ by the relation $F_{P}(H):=P\left(H-H R_{\bar{P}}(H) H\right) P$ on the domain

$$
\operatorname{Dom}\left(F_{P}\right)=
$$

$\left\{H: X \rightarrow X \mid H_{\bar{P}}\right.$ is invertible, $\left.\operatorname{Ran} P \subseteq \operatorname{Dom}(H), \operatorname{Ran} R_{\bar{P}}(H) \subseteq \operatorname{Dom}(P H \bar{P})\right\}$.

A key property of the maps $F_{P}$ is given in the following statement proven in [7]:

Theorem 11.1 (Isospectrality theorem). (i) $0 \in \sigma(H) \Longleftrightarrow 0 \in \sigma\left(F_{P}(H)\right)$,

(ii) $H \psi=0 \Longleftrightarrow F_{P}(H) \varphi=0$ with $\varphi=P \psi(" \Rightarrow ")$ and $\psi=\left(\mathbf{1}-R_{\bar{P}}(H) H\right) \varphi$ $(" \Leftarrow ")$.

Let $P_{e \rho}$ be defined as

$$
P_{e \rho}:=\chi_{L_{p}=e} \otimes \chi_{M_{\theta} \leq \rho},
$$

where $\chi_{L_{p}=e}$ is the eigenprojection for the operator $L_{p}$ corresponding to an eigenvalue $e \in \sigma\left(L_{p}\right)$ and $\chi_{M_{\theta} \leq \rho}$ is the spectral projection for the self-adjoint operator $M_{\theta}:=\operatorname{Im} L_{0, \theta}$ corresponding to the spectral interval $[0, \rho]$ (remember that $M_{\theta}$ is a positive operator).

The following result is proven in [25], Lemma 6.3.

Lemma 11.2. Assume Condition (B). Take $\rho_{0} \in(0, \sigma / 2)$ and let $|g|<\sqrt{\rho_{0}} g_{0}$, where $g_{0}$ is given after (3.9). If $z \in S_{e}$ then $K_{\theta z}:=K_{\theta}-z \in \operatorname{Dom}\left(F_{P_{e \rho_{0}}}\right)$, and the operator $K_{\theta z}^{(1)}:=F_{P_{e \rho_{0}}}\left(K_{\theta z}\right)$ acting on $\operatorname{Ran} P_{e \rho_{0}}$ is of the form

$$
K_{\theta z}^{(1)}=(e-z) \mathbf{1}+L_{r \theta}+g^{2} \Lambda_{e}+O\left(\epsilon\left(g, \rho_{0}\right)\right) .
$$


The remainder is estimated in operator norm, $\left\|O\left(\epsilon\left(g, \rho_{0}\right)\right)\right\| \leq C \epsilon\left(g_{\delta \beta}, \rho_{0}\right)$, where $g_{\delta \beta}=|g| \max _{j} \sup _{|\theta|<\theta_{0}}\left\|G_{j}\right\|_{\delta \beta_{j}, 1 / 2, \theta}$, with a $C$ independent of $\delta \beta_{j}, \theta$, and where we have set

$$
\epsilon(g, \rho):=|g| \rho^{\mu}+|g|^{3} \rho^{-1 / 2}+|g|^{2} \rho^{2 \mu-1} .
$$

To unify the following analysis we write $\Omega_{\theta}^{\#}$ for either $\Omega_{\theta}$ or $\Omega_{\frac{\theta}{\theta}}^{*}$. Correspondingly, $I_{\theta}^{\#}, L_{0 \theta \#}$ and $K_{\theta}^{\#}$ stand for either $I_{\theta}$ or $I_{\bar{\theta}}^{*}$, for either $L_{0 \theta}$ or $L_{0 \bar{\theta}}$, and for either $K_{\theta}$ or $K_{\frac{\theta}{*}}^{*}$, respectively. We use the shorthand $P_{0} \equiv P_{0 \rho_{0}}$ and $\bar{R}_{0}(A):=\bar{P}_{0} A_{\bar{P}_{0}}^{-1} \bar{P}_{0}$, where $A_{P}:=P A P$. We will assume that

$$
\tau^{\prime} \gg g^{2+\alpha}, \quad \alpha=\frac{\mu-\frac{1}{2}}{\mu+\frac{1}{2}} .
$$

Theorem 11.1 and Lemma 11.2 imply that $K_{\theta}^{\#} \in \operatorname{Dom}\left(F_{P_{0}}\right)$, that

$$
F_{P_{0}}\left(K_{\theta}^{\#}\right) P_{0} \Omega_{\theta}^{\#}=0,
$$

and that the original eigenvector $\Omega_{\theta}^{\#}$ can be reconstructed as

$$
\Omega_{\theta}^{\#}=\left[\mathbf{1}-g \bar{R}_{0}\left(K_{\theta}^{\#}\right) I_{\theta}^{\#}\right] P_{0} \Omega_{\theta}^{\#} .
$$

We expand $\bar{R}_{0}\left(K_{\theta}^{\#}\right)$ in this expression into a Neumann series,

$$
\Omega_{\theta}^{\#}=\sum_{n=0}^{N-1} g^{n}\left(-\bar{R}_{0}\left(L_{0 \theta^{\#}}\right) I_{\theta}^{\#}\right)^{n} P_{0} \Omega_{\theta}^{\#}+O\left(\left(g_{\delta \beta} \rho_{0}^{-1 / 2}\right)^{N}\right),
$$

for any $N \geq 1$, provided that $O\left(\left(g_{\delta \beta} \rho_{0}^{-1 / 2}\right)\right)=o_{g}(1)$. The remainder term in (11.7) is obtained by using a standard estimate on the $N$ th term of the convergent Neumann series. Indeed, writing

$$
\begin{aligned}
& {\left[\bar{R}_{0}\left(L_{0 \theta^{\#}}\right) I_{\theta}^{\#}\right]^{N} } \\
= & \left(M_{\theta}+\rho_{0}\right)^{-1 / 2}\left[\frac{M_{\theta}+\rho_{0}}{L_{0 \theta^{\#}} \bar{P}_{0}} \bar{P}_{0}\left(M_{\theta}+\rho_{0}\right)^{-1 / 2} I_{\theta}^{\#}\left(M_{\theta}+\rho_{0}\right)^{-1 / 2}\right]^{N}\left(M_{\theta}+\rho_{0}\right)^{1 / 2}
\end{aligned}
$$

and using the estimates

$$
|g|\left\|\left(M_{\theta}+\rho_{0}\right)^{-1 / 2} I_{\theta}^{\#}\left(M_{\theta}+\rho_{0}\right)^{-1 / 2}\right\| \leq g_{\delta \beta} \rho_{0}^{-1 / 2}
$$

(see also Lemma 5.3 of [25]) and $\left\|\frac{M_{0}+\rho_{0}}{L_{0 \theta} \# \bar{P}_{0}} \bar{P}_{0}\right\| \leq C$, we obtain

$$
|g|^{N}\left\|\left[\bar{R}_{0}\left(L_{0 \theta \#}\right) I_{\theta}^{\#}\right]^{N} P_{0} \Omega_{\theta}^{\#}\right\| \leq \rho_{0}^{-1 / 2}\left(g_{\delta \beta} \rho_{0}^{-1 / 2}\right)^{N} \rho_{0}^{1 / 2} .
$$

Observe that since $g_{\delta \beta} \rho_{0}^{-1}=o_{g}(1)$ we have

$$
\Omega_{\theta}^{\#}=\sum_{n=0,1} g^{n}\left(-\bar{R}_{0}\left(L_{0 \theta \#}\right) I_{\theta}^{\#}\right)^{n} P_{0} \Omega_{\theta}^{\#}+o(g) .
$$


Let $K^{\#(1)}:=F_{P_{0}}\left(K_{\theta}^{\#}\right)$. As in (11.2) it can be written as

$$
K^{\#(1)}=K_{0}^{\#(1)}+W^{\#},
$$

where $K_{0}^{\#(1)}:=L_{r \theta \#}+g^{2} \Lambda^{\#}$ with $\Lambda^{\#}$ either $\Lambda_{0}$ or $\Lambda_{0}^{*}$, where $\Lambda_{0}$ is given by $(7.1)$ with $e=0$. We assume that $\delta \beta$ varies in the set $|\delta \beta| \leq c$, for some $c>0$, so that $g_{\delta \beta}$ can be replaced by $g$. Take $\rho_{0}=g^{2-2 \alpha}$ with $\alpha=\frac{\mu-1 / 2}{\mu+1 / 2}$, then Lemma 11.2 gives

$$
W^{\#}=O\left(g^{2+\alpha}\right) \text {. }
$$

By Theorem 11.1,

$$
K^{\#(1)} P_{0} \Omega_{\theta}^{\#}=0 .
$$

Let $Q_{0}^{\#}:=\chi_{K_{0}^{\#(1)}=0}=\chi_{\Lambda \#=0} \otimes \chi_{L_{r}=0}$, where $\chi_{\Lambda \#=0}$ is the Riesz projection onto the kernel of $\Lambda^{\#}$. The operator $K_{0}^{\#(1)}$ is normal with the simple eigenvalue 0 separated from the rest of the spectrum by a gap $\geq c \min \left(\tau^{\prime}, g^{2}\right)$ for some $c>0$. The operator $K^{\#(1)}$ has also the simple eigenvalue 0 , which, by the Kato-Rellich theorem, (11.12) and the assumption (11.4), is separated from the rest of the spectrum by a gap $\geq c^{\prime} \min \left(\tau^{\prime}, g^{2}\right)$ for some $c^{\prime}>0$. Hence, we conclude that $K^{\#(1)} \in \operatorname{Dom}\left(F_{Q_{0}^{\#}}\right)$. Therefore, by Theorem 11.1,

$$
P_{0} \Omega_{\theta}^{\#}=\left(\mathbf{1}-R \overline{Q_{0}^{\#}}\left(K^{\#(1)}\right) W^{\#}\right) Q_{0}^{\#} \Omega_{\theta}^{\#} .
$$

Since $\left\|R \overline{Q_{0}^{\#}}\left(K^{\#(1)}\right)\right\| \leq C\left[\min \left(\tau^{\prime}, g^{2}\right)\right]^{-1}$ we have the absolutely convergent perturbation expansion

$$
P_{0} \Omega_{\theta}^{\#}=C^{\#} \sum_{n=0}^{\infty}\left(-R \frac{}{Q_{0}^{\#}}\left(K_{0}^{\#(1)}\right) W^{\#}\right)^{n} \zeta^{\#} \otimes \Omega_{r} .
$$

Here, $\zeta^{\#} \in \operatorname{Ran} \chi_{L_{p}=0}$ is the unique vector in the kernel of $\Lambda^{\#}$, normalized as

$$
\Lambda^{\#} \zeta^{\#}=0, \quad\|\zeta\|=1, \quad\left\langle\zeta^{*}, \zeta\right\rangle=1 .
$$

(Recall that $\Lambda^{\#}$ is either $\Lambda_{0}$ or $\Lambda_{0}^{*}$, so $\zeta^{\#}$ is either a null vector, $\zeta$, of $\Lambda_{0}$ or a null vector, $\zeta^{*}$, of $\Lambda_{0}^{*}$.) Letting $\zeta^{\# \#}$ equal $\zeta$ if $\#=*$ and letting it equal $\zeta^{*}$ otherwise, the constant in (11.15) takes the form $C^{\#}=\left\langle\zeta^{\# \#} \otimes \Omega_{r}, \Omega_{\theta}^{\#}\right\rangle$.

The overlap $\left\langle\zeta^{*}, \zeta\right\rangle$ can be chosen strictly positive since the $\zeta^{\#}$ are the Perron-Frobenius eigenvectors of $\Lambda^{\#}$ (i.e., their components can be chosen nonnegative), and every component of $\zeta$ is strictly positive (see below). The last relation in (11.16) is then achieved by scaling $\zeta^{*}$ properly. The normalization $\left\langle\Omega_{\theta}^{*}, \Omega_{\theta}\right\rangle=1$ together with (11.7), (11.12) (11.15) and (11.16) implies that

$$
\overline{C^{*}} C=1+o(g) \text {. }
$$

If the condition (11.4), $\tau^{\prime} \gg g^{2+\alpha}$, does not hold then we have to apply the Feshbach map iteratively and use a corresponding perturbation theory for eigenvectors. We omit here this analysis and refer the reader to [5-7] for general references on such a RG perturbation theory and we will present elsewhere the RG perturbation theory in our specific case. 
In Section 10 we have shown that Null $\Lambda=\mathbb{C} \Omega_{p}$ and consequently $\zeta=\Omega_{p}$ and the vector $\Omega_{0}=\zeta \otimes \Omega_{r}=\Omega_{p} \otimes \Omega_{r}$ is our unperturbed state introduced in Section 6. Recall that $\Omega_{p}=\Omega_{p}^{\beta_{p}}$ is the particle Gibbs state at temperature $\beta_{p}$.

Expressions (10.1)-(10.2) for $\Lambda_{0}$ imply the following relation among vectors $\zeta^{*}$ corresponding to different particle temperatures

$$
\zeta^{*}=\left.\sqrt{\frac{\operatorname{Tr} \mathrm{e}^{-\beta_{p} H_{p}}}{N}}\left(e^{\beta_{p} H_{p} / 2} \otimes \mathbb{1}_{p}\right) \zeta^{*}\right|_{\beta_{p}=0} .
$$

In view of (11.18), it suffices to consider $\beta_{p}=0$.

In general there is no simple expression for the eigenvector $\zeta^{*}$. However, there are three cases where such an expression can be obtained. We expand $\left.\zeta^{*}\right|_{\beta_{p}=0}$ in the basis $\varphi_{j} \otimes \varphi_{j}$ as

$$
\left.\zeta^{*}\right|_{\beta_{p}=0}=\sum_{j} \gamma_{j} \varphi_{j} \otimes \varphi_{j} .
$$

Here $\varphi_{j}$ and $E_{j}$ are defined after (2.37). Note that the normalization condition and (2.37) imply that $\sum_{j} \gamma_{j}=\sqrt{N}$. We have the following results:

(i) If $\beta_{1}=\beta_{2}=\beta$ and $\beta_{p}=0$, then

$$
\gamma_{j}=\frac{\sqrt{N}}{\operatorname{Tr} e^{-\beta H_{p}}} e^{-\beta E_{j}} .
$$

(ii) If $\beta_{1}$ is fixed and $\beta_{2} \rightarrow 0$, and $\beta_{p}=0$, then

$$
\gamma_{j}=\frac{1}{\sqrt{N}}+O\left(\beta_{2}\right)
$$

(iii) If $N=\operatorname{dim} \mathcal{H}_{p}=2$ and $\beta_{p}=0$, then

$$
\gamma_{1}=\frac{\sqrt{2} \alpha(E)}{\alpha(E)+1} \quad \text { and } \quad \gamma_{2}=\frac{\sqrt{2}}{\alpha(E)+1},
$$

where

$$
\alpha(E)=1+\frac{\sum_{j=1,2} g_{j}(E)}{\sum_{j=1,2} g_{j}(E) \rho_{j}(E)} .
$$

Here, we use the notation $E:=E_{2}-E_{1}, \rho_{j}(E):=\frac{1}{e^{\beta_{j} E}-1}$ and

$$
g_{j}(E):=\int_{|k|=|E|}\left[\left|G_{j}(k)_{12}\right|^{2}+\left|G_{j}(k)_{21}\right|^{2}\right] d k .
$$

Equation (11.20) follows from (10.1), (10.3) and (10.4). The expressions (11.22)(11.23) come simply from solving a two-dimensional eigenvalue problem. Equation (11.21) follows from a straightforward perturbation theory in $\beta_{2}$. See Appendix D for an outline of the proofs. 


\section{Entropy production rate for $\eta$}

In this section we prove Theorem 3.2. Recall that the stationary state $\eta$ mentioned in this theorem is, in fact, given in (9.10). To analyze the entropy production, $E P(\eta)$, in this state $\eta$ we use expression $(2.24)$,

$$
\operatorname{EP}(\eta)=\left(\beta_{1}-\beta_{2}\right) \eta\left(\phi_{1}\right)
$$

which relates it to the heat flow, $\eta\left(\phi_{1}\right)$, in the state $\eta$. Recall that

$$
\phi_{1}=i g\left[v_{1}, H_{r 1}\right]=i g \eta\left(a_{1}\left(\omega G_{1}\right)-a_{1}^{*}\left(\omega G_{1}\right)\right) .
$$

If $\beta_{1}=\beta_{2}$, then $\eta\left(\phi_{1}\right)=0$, c.f. [19]. We want to show here that $\eta\left(\phi_{1}\right)>0$ if $\beta_{1}>\beta_{2}$. Our proof is based on

Theorem 12.1. Set $\beta=\max \left(\beta_{1}, \beta_{2}\right)$ and let $\Omega_{0}^{*}=\zeta^{*} \otimes \Omega_{r}$ with $\Lambda^{*} \zeta^{*}=0$ (see (11.16)) and with the vector $\Omega_{0}$ defined in (2.37). Under the conditions of Theorem 3.1, we have

$$
\eta\left(\phi_{1}\right)=g^{2} \eta^{\prime}+o\left(g^{2}\right) O(\delta \beta),
$$

where, recall, $\delta \beta:=\beta_{1}-\beta_{2}$ and

$$
\eta^{\prime}=2 \operatorname{Re}\left\langle\Omega_{0}^{*}, \pi\left(v_{1}\right) L_{r 1} i\left(L_{0}+i 0\right)^{-1} e^{-\beta L_{0} / 2} \pi\left(v_{1}\right) \Omega_{0}\right\rangle .
$$

Moreover, we have the explicit expression

$$
\eta^{\prime}=\frac{2 \pi}{\sqrt{N}} \sum_{j>i}\left(\gamma_{j} e^{\beta_{1} E_{j i}}-\gamma_{i}\right) \frac{E_{j i} g_{j i}\left(E_{j i}\right)^{2}}{e^{\beta_{1} E_{j i}}-1},
$$

where $E_{j i}=E_{j}-E_{i}, g_{j i}(E)^{2}=\int_{\mathbb{R}^{3}} d^{3} k\left|\left\langle\varphi_{j}, G_{1}(k) \varphi_{i}\right\rangle\right|^{2} \delta\left(E_{j i}-\omega\right)$. The numbers $\gamma_{j} \geq 0$ are the components of the vector $\zeta^{*}$, see (11.19), normalized as in (11.16), at $\beta_{p}=0$. Observe that by (11.20), $\eta^{\prime}=0$ for $\beta_{1}=\beta_{2}$.

The following result shows that $\eta^{\prime}$ is strictly positive for small nonzero temperature differences.

Theorem 12.2. If $\delta \beta=\beta_{1}-\beta_{2}>0$ is small and the coupling functions (2.4) satisfy $G=G_{1}=G_{2}$ then the linear term of $\eta^{\prime}$ in $\delta \beta$ is

$$
\frac{\delta \beta}{2} \frac{Z_{p}\left(\beta_{p}\right)}{Z_{p}\left(\beta_{1}+\beta_{p} / 2\right)} \sum_{j>k} \frac{E_{j k}^{2} g_{j k}\left(E_{j k}\right)^{2}}{e^{\beta_{1} E_{j}}-e^{\beta_{1} E_{k}}}>0,
$$

where $Z_{p}(\beta)=\operatorname{tr} e^{-\beta H_{p}}$ is the particle partition function.

Remark. Using (11.22) for the two-dimensional case it is easy to verify that the linear term of $\eta^{\prime}$ in $\delta \beta$ is strictly positive (without the assumption $G_{1}=G_{2}$ ), see the proof of Theorem 3.2 below which gives also an explicit expression for $\eta^{\prime}$. Also, in the general case, if $G_{1}$ is close to $G_{2}$ one deduces strict positivity of $\eta^{\prime}$ in the linear term in $\delta \beta$ by a perturbation argument. 
Proof of Theorem 12.1. To simplify the exposition we restrict ourselves to the case $\tau^{\prime} \gg g^{2+\alpha}$, for $\alpha=\frac{\mu-1 / 2}{\mu+1 / 2}$, and $\mu>3 / 2$. Pick $\rho_{0}=g^{2-2 \alpha}$. First we prove an estimate on $\eta\left(\phi_{1}\right)$ which is rougher than (12.3) and then we explain how to obtain (12.3). Recall that

$$
\eta\left(\phi_{1}\right)=\left\langle\Omega_{\frac{*}{\theta}}, \pi\left(\phi_{1}\right)_{\theta} \Omega_{\theta}\right\rangle .
$$

Let $\Omega_{0}^{\#}=\zeta^{\#} \otimes \Omega_{r}$ and take a number $M$ so large that $\left(g^{2+\alpha}\left[\min \left(\tau^{\prime}, g^{2}\right)\right]^{-1}\right)^{M}=$ $o(g)$. Substituting expansions (11.7) and (11.15) into the r.h.s. of this expression, using that $\left(g_{\delta \beta} \rho_{0}^{-1 / 2}\right)^{2}=o(g)$, for $\rho_{0}=g^{2-2 \alpha}, \alpha>1 / 2$, and using that $\phi_{1}$ is proportional to $g$, we find

$$
\eta\left(\phi_{1}\right)=\eta_{0}+\eta_{1}+\operatorname{Rem}+o\left(g^{2}\right),
$$

where

$$
\begin{aligned}
\eta_{0}:= & \left\langle\Omega_{0}^{*}, \pi\left(\phi_{1}\right) \Omega_{0}\right\rangle \\
\eta_{1}:= & \left.-g\left\langle\Omega_{0}^{*},\left[I_{\theta} \bar{R}_{0}\left(L_{0 \theta}\right) \pi\left(\phi_{1}\right)_{\theta}+\pi\left(\phi_{1}\right)_{\theta} \bar{R}_{0}\left(L_{0 \theta}\right) I_{\theta}\right)\right] \Omega_{0}\right\rangle \\
\text { Rem }= & \sum_{m+n \geq 1}^{M} \sum_{k, l=0}^{1} g^{k+l}\left\langle\left(-\bar{R}_{0}\left(L_{0 \bar{\theta}}\right) I_{\bar{\theta}}^{*}\right)^{k}\left(-R_{\overline{Q_{0}^{*}}}\left(K_{0}^{\#(1)}\right) W^{*}\right)^{m} \Omega_{0}^{*}\right. \\
& \left.\pi\left(\phi_{1}\right)_{\theta}\left(-\bar{R}_{0}\left(L_{0 \theta}\right) I_{\theta}\right)^{l}\left(-R_{\bar{Q}_{0}}\left(K_{0}^{(1)}\right) W\right)^{n} \Omega_{0}\right\rangle .
\end{aligned}
$$

Here we replaced on the r.h.s the factor $\overline{C^{*}} C=1+o(g)$ by 1 (see (11.15) and (11.17)). Using the pull-through procedure and elementary estimates of the resulting integrals we obtain that

$$
\operatorname{Rem}=o\left(g^{2}\right) .
$$

Since $\phi_{1}$ is linear in creation and annihilation operators, see (12.1), we have

$$
\eta_{0}=0 \text {. }
$$

It remains to compute $\eta_{1}$. Using

$$
\left(\bar{P}_{\rho_{0}}-\mathbf{1}\right) I_{\theta}^{\#} \Omega_{0}^{\#}=O\left(\rho_{0}\right)
$$

([25] Lemma 5.3), removing the spectral deformation and using that $\pi\left(\phi_{1}\right)=$ $g\left[\pi\left(v_{1}\right), i L_{r 1}\right]$, we obtain $\eta_{1}=g^{2} \eta^{\prime}+o\left(g^{2}\right)$, where

$$
\eta^{\prime}=-\left\langle\Omega_{0}^{*},\left[\pi\left(v_{1}\right) i L_{r 1}\left(L_{0}+i 0\right)^{-1} I-I\left(L_{0}+i 0\right)^{-1} i L_{r 1} \pi\left(v_{1}\right)\right] \Omega_{0}\right\rangle .
$$

Next, note that the contribution of the $v_{2}$-part of $I$ to $\eta^{\prime}$ is zero since the resulting expression is linear in creation and annihilation operators for the first and second reservoirs separately. The contribution of the $\pi\left(v_{1}\right)$-part of $I$ is also zero by the symmetry of (12.15). Hence we have $\eta^{\prime}=A-B$, where

$$
\begin{aligned}
& A=\left\langle\Omega_{0}^{*}, \pi\left(v_{1}\right) i L_{r 1}\left(L_{0}+i 0\right)^{-1} \pi^{\prime}\left(\gamma^{i / 2}\left(v_{1}\right)\right) \Omega_{0}\right\rangle, \\
& B=\left\langle\Omega_{0}^{*}, \pi^{\prime}\left(\gamma^{i / 2}\left(v_{1}\right)\right) i L_{r 1}\left(L_{0}+i 0\right)^{-1} \pi\left(v_{1}\right) \Omega_{0}\right\rangle .
\end{aligned}
$$


Using that $\pi^{\prime}\left(\gamma^{i / 2}\left(v_{1}\right)\right) \Omega_{0}=J e^{\left(\beta L_{0}-\tilde{L}\right) / 2} \pi\left(v_{1}\right) \Omega_{0}$, where $\tilde{L}$ is given after (4.19), and the fact that $J e^{-\tilde{L} / 2} \pi\left(v_{1}\right) \Omega_{0}=\pi\left(v_{1}^{*}\right) \Omega_{0}=\pi\left(v_{1}\right) \Omega_{0}$, we transform

$$
A=\left\langle\Omega_{0}^{*}, \pi\left(v_{1}\right) i L_{r 1}\left(L_{0}+i 0\right)^{-1} e^{-\beta L_{0} / 2} \pi\left(v_{1}\right) \Omega_{0}\right\rangle .
$$

We use the relations $\pi^{\prime}\left(\gamma^{i / 2}\left(v_{1}\right)\right)=J e^{\left(\beta L_{0}-\tilde{L}\right) / 2} \pi\left(v_{1}\right) e^{-\left(\beta L_{0}-\tilde{L}\right) / 2} J$ and

$$
J i L_{r 1}\left(L_{0}+i 0\right)^{-1} \pi\left(v_{1}\right) \Omega_{0}=-i L_{r 1}\left(L_{0}+i 0\right)^{-1} e^{-\tilde{L} / 2} \pi\left(v_{1}\right) \Omega_{0},
$$

see also (5.1), to find that

$$
B=-\left\langle\Omega_{0}^{*}, J e^{\left(\beta L_{0}+\tilde{L}\right) / 2} \pi\left(v_{1}\right) i L_{r 1}\left(L_{0}+i 0\right)^{-1} e^{-\beta L_{0} / 2} \pi\left(v_{1}\right) \Omega_{0}\right\rangle .
$$

Finally, since $\langle J u, J v\rangle=\overline{\langle u, v\rangle}, J \Omega_{0}^{*}=\Omega_{0}^{*}, L_{p} \Omega_{0}^{*}=0$ and $\left(\beta L_{0}-\bar{L}\right) \Omega_{0}^{*}=0$ we obtain

$$
\bar{B}=-\left\langle\Omega_{0}^{*}, \pi\left(v_{1}\right) i L_{r 1}\left(L_{0}+i 0\right)^{-1} e^{-\beta L_{0} / 2} \pi\left(v_{1}\right) \Omega_{0}\right\rangle=-A .
$$

Since $\eta^{\prime}=A-B$ this gives (12.4). that

Collecting estimates (12.8), (12.12), (12.13) and $\eta_{1}=g^{2} \eta^{\prime}+o\left(g^{2}\right)$ we find

$$
\eta\left(\phi_{1}\right)=g^{2} \eta^{\prime}+o\left(g^{2}\right)
$$

where $\eta^{\prime}$ is given by (12.4). This proves the rough version of (12.3)-(12.4).

Before we refine estimate (12.16) let us show (12.5). We expand the vectors $\Omega_{0}^{*}$ and $\Omega_{0}$ in the basis $\varphi_{j j} \otimes \Omega_{r}, \varphi_{j j}=\varphi_{j} \otimes \varphi_{j}$,

$$
\Omega_{0}=\sum_{j=1}^{n} \alpha_{j} \varphi_{j j} \otimes \Omega_{r}, \quad \Omega_{0}^{*}=\sum_{j=1}^{n} \tilde{\gamma}_{j} \varphi_{j j} \otimes \Omega_{r}
$$

with $\alpha_{j} \geq 0, \sum_{j} \alpha_{j}^{2}=1$ and $\tilde{\gamma}_{j} \geq 0, \sum_{j} \alpha_{j} \tilde{\gamma}_{j}=1$. Plugging the expressions in (12.17) into the r.h.s. of (12.4), using (2.35) in order to express $\pi\left(v_{1}\right)$ in terms of creation and annihilation operators, $a_{\ell 1}^{\#}$ and $a_{r 1}^{\#}$,

$$
\pi\left(v_{1}\right)=a_{\ell 1}\left(\sqrt{1+\rho_{1}} G_{1 \ell}\right)+a_{r 1}\left(\sqrt{\rho_{1}} G_{1 \ell}\right)+\text { h.c. },
$$

where $\rho_{1}=\left(e^{\beta_{1} \omega}-1\right)^{-1}$, pulling through the annihilation operators to the right and using that $\pi\left(v_{1}\right)$ (or $G_{1 \ell}$ ) acts only on the first (left) factor in $\varphi_{j j}=\varphi_{j} \otimes \varphi_{j}$, we obtain

$$
\begin{aligned}
\eta^{\prime}= & -2 \sum_{j} \alpha_{j} \tilde{\gamma}_{j} \operatorname{Im} \\
& \times \int\left\{\left(1+\rho_{1}\right) \omega\left\langle\varphi_{j}, G_{1}^{*}\left(H_{p}-E_{j}+\omega+i 0\right)^{-1} e^{-\beta\left(H_{p}-E_{j}+\omega\right) / 2} G_{1} \varphi_{j}\right\rangle\right. \\
& \left.-\rho_{1} \omega\left\langle\varphi_{j}, G_{1}^{*}\left(H_{p}-E_{j}-\omega+i 0\right)^{-1} e^{-\beta\left(H_{p}-E_{j}-\omega\right) / 2} G_{1}^{*} \varphi_{j}\right\rangle\right\} d^{3} k .
\end{aligned}
$$

Inserting the partition of unity $\mathbf{1}=\sum_{j}\left|\varphi_{j}\right\rangle\left\langle\varphi_{j}\right|$ into the inner products on the r.h.s. we obtain furthermore

$$
\eta^{\prime}=2 \pi \sum_{i, j} \alpha_{i} \tilde{\gamma}_{i} \int \omega\left|\left\langle\varphi_{i}, G_{1} \varphi_{j}\right\rangle\right|^{2}\left\{\left(1+\rho_{1}\right) \delta\left(E_{j i}+\omega\right)-\rho_{1} \delta\left(E_{j i}-\omega\right)\right\} d^{3} k
$$


Interchanging the labels in the sum of the first term and noticing that in the resulting expression the integrals vanish unless $E_{j i}>0$, i.e., $E_{j}>E_{i}$, or $j>i$, we arrive at

$$
\eta^{\prime}=2 \pi \sum_{j>i}\left(\alpha_{j} \tilde{\gamma}_{j} e^{\beta_{1} E_{j i}}-\alpha_{i} \tilde{\gamma}_{i}\right) E_{j i} g_{j i}\left(E_{j i}\right)^{2}\left(e^{\beta_{1} E_{j i}}-1\right)^{-1}
$$

where $g_{j i}(E)^{2}:=\int_{\mathbb{R}^{3}} d^{3} k\left|\left\langle\varphi_{j}, G_{1}(k) \varphi_{i}\right\rangle\right|^{2} \delta\left(E_{j i}-\omega\right)$. Observing that, due to (11.18), $\alpha_{i} \tilde{\gamma}_{i}=\left.N^{-1 / 2} \tilde{\gamma}_{i}\right|_{\beta_{p}=0} \equiv N^{-1 / 2} \gamma_{i}$, we arrive at (12.5).

Since $\eta^{\prime}=O(\delta \beta)$ (the energy flow vanishes if $\beta_{1}=\beta_{2}$ ) estimate (12.16) is ineffective if $\delta \beta$ is so small that $\delta \beta g^{2}=o\left(g^{2}\right)$. However, with a little bit more work (12.16) can be upgraded to estimate (12.3). We sketch a proof of this estimate without going into much detail. We begin with some notation.

Consider the self-adjoint Liouville operator for equal reservoir temperatures $\beta_{2}=\beta_{1}=\beta$,

$$
\tilde{L}=L_{0}+g \tilde{I}
$$

where

$$
\tilde{I}=\tilde{\pi}(v)-\tilde{\pi^{\prime}}(v)
$$

with $\tilde{\pi}=\left.\pi\right|_{\beta_{1}=\beta_{2}=\beta}$ and similarly for $\widetilde{\pi}^{\prime}$. Define $\widetilde{\Omega}=\frac{e^{-\beta \tilde{L}^{(l)} / 2} \Omega_{0}}{\| e^{-\beta \tilde{L}^{(l)} / 2 \Omega_{0} \|}}$, where $\widetilde{L}^{(l)}=$ $L_{0}+\widetilde{\pi}(v)$. Since the entropy production does not depend on $\beta_{p}$ we set from now on $\beta_{p}=\beta_{1}$. The operator $\left.K\right|_{\beta_{p}=\beta_{1}=\beta_{2}=\beta}=\tilde{L}$ is selfadjoint, (5.11), and hence $\widetilde{\Omega}_{\bar{\theta}}^{*}=\widetilde{\Omega}_{\bar{\theta}}$. From (12.7) we obtain

$$
\begin{aligned}
\eta\left(\phi_{1}\right)= & \left\langle\left(\Omega_{\bar{\theta}}^{*}-\widetilde{\Omega}_{\bar{\theta}}\right), \pi\left(\phi_{1}\right)_{\theta} \Omega_{\theta}\right\rangle+\left\langle\widetilde{\Omega}_{\bar{\theta}}, \pi\left(\phi_{1}\right)_{\theta}\left(\Omega_{\theta}-\widetilde{\Omega}_{\theta}\right)\right\rangle \\
& +\left\langle\widetilde{\Omega}_{\bar{\theta}}, \pi\left(\phi_{1}\right)_{\theta} \widetilde{\Omega}_{\theta}\right\rangle
\end{aligned}
$$

We consider the last term first. Recall that $\phi_{1}=g\left[v, i H_{r 1}\right]=g\left[v_{1}, i H_{r 1}\right]$ and therefore $\pi\left(\phi_{1}\right)=\widetilde{\pi}\left(\phi_{1}\right)$. It follows that

$$
\left\langle\widetilde{\Omega}_{\bar{\theta}}, \pi\left(\phi_{1}\right)_{\theta} \widetilde{\Omega}_{\theta}\right\rangle=\left\langle\widetilde{\Omega}_{\bar{\theta}}, \widetilde{\pi}\left(\phi_{1}\right)_{\theta} \widetilde{\Omega}_{\theta}\right\rangle=\left\langle\widetilde{\Omega}, \widetilde{\pi}\left(\phi_{1}\right) \widetilde{\Omega}\right\rangle .
$$

Note that the r.h.s. of (12.21) describes the heat flow into reservoir $r 1$ for the equal temperature system. Since the heat flows vanish individually in the equal temperature case we have shown that the last term in (12.20) vanishes.

To estimate the first two terms on the r.h.s. we use as before expansions (11.7) and (11.15) for $\Omega_{\theta}^{\#}=\Omega_{\theta}, \Omega_{\frac{*}{\theta}}$ and similar expansions (obtained by setting $\beta_{2}=$ $\beta_{1}=\beta$ in (11.7) and (11.15)) for $\widetilde{\Omega}_{\theta}$. As a result we obtain an expression for (12.20) of the type (12.8)-(12.11) but with some of the powers in Rem, (12.11), replaced by the differences, e.g., $\left(-\bar{R}_{0}\left(L_{0 \theta}\right) I_{\theta}\right)^{l}-\left(-\bar{R}_{0}\left(L_{0 \theta}\right) \widetilde{I}_{\theta}\right)^{l}$ or $\left(R_{\bar{Q}_{0}}\left(K_{0}^{(1)}\right) W\right)^{n}-$ 
$\left.\left(R_{\bar{Q}_{0}}\left(K_{0}^{(1)}\right) W\right)^{n}\right|_{\beta_{2}=\beta_{1}=\beta}$. These differences are estimated by using a telescopic expansion, e.g.,

$$
\begin{aligned}
& \left(-\bar{R}_{0}\left(L_{0 \theta}\right) I_{\theta}\right)^{l}-\left(-\bar{R}_{0}\left(L_{0 \theta}\right) \widetilde{I}_{\theta}\right)^{l} \\
& \quad=\sum_{j=1}^{l}\left(-\bar{R}_{0}\left(L_{0 \theta}\right) I_{\theta}\right)^{j-1}\left(-\bar{R}_{0}\left(L_{0 \theta}\right)\right)\left(I_{\theta}-\widetilde{I}_{\theta}\right)\left(-\bar{R}_{0}\left(L_{0 \theta}\right) \widetilde{I}_{\theta}\right)^{l-j},
\end{aligned}
$$

and then estimating the first type of the differences in norm while for the second type we do first the pull-through and contraction procedure and then estimate the resulting integrals. As a result we have

$$
\begin{aligned}
\eta\left(\phi_{1}\right)= & -g\left\langle\Omega_{0}^{*}-\Omega_{0}, \pi\left(\phi_{1}\right)_{\theta} \bar{R}_{0}\left(L_{0, \theta}\right) I_{\theta} \Omega_{0}\right\rangle \\
& -g\left\langle\bar{R}_{0}\left(L_{0, \bar{\theta}}\right)\left[I_{\bar{\theta}}^{*} \Omega_{0}^{*}-\widetilde{I}_{\bar{\theta}} \Omega_{0}\right], \pi\left(\phi_{1}\right)_{\theta} \Omega_{0}\right\rangle \\
& -g\left\langle\Omega_{0}, \pi\left(\phi_{1}\right)_{\theta} \bar{R}_{0}\left(L_{0, \theta}\right)\left[I_{\theta}-\widetilde{I}_{\theta}\right] \Omega_{0}\right\rangle \\
& +o\left(g^{2}\right) O(\delta \beta) \\
= & \eta_{1}+\eta_{2}+o\left(g^{2}\right) O(\delta \beta)
\end{aligned}
$$

where $\eta_{1}$ is given in (12.10) and

$$
\eta_{2}=g\left\langle\Omega_{0},\left[\widetilde{I}_{\theta} \bar{R}_{0}\left(L_{0, \theta}\right) \pi\left(\phi_{1}\right)_{\theta}+\pi\left(\phi_{1}\right)_{\theta} \bar{R}_{0}\left(L_{0, \theta}\right) \widetilde{I}_{\theta}\right] \Omega_{0}\right\rangle .
$$

Since the contribution of the $v_{2}$-component of $\widetilde{I}_{\theta}$ is zero we can omit the tilde $(\sim)$ in (12.24). Thus the expression for $\eta_{2}$ coincides up to the sign and the substitution $\Omega_{0}^{*} \rightarrow \Omega_{0}$ with the expression (12.10) for $\eta_{1}$, i.e.

$$
\eta_{1}+\eta_{2}=-g\left\langle\Omega_{0}^{*}-\Omega_{0},\left[I_{\theta} \bar{R}_{0}\left(L_{0, \theta}\right) \pi\left(\phi_{1}\right)_{\theta}+\pi\left(\phi_{1}\right)_{\theta} \bar{R}_{0}\left(L_{0, \theta}\right) I_{\theta}\right] \Omega_{0}\right\rangle .
$$

We proceed with the r.h.s. of (12.25) exactly as we did above with $\eta_{1}$ alone in equation (12.14), and we arrive at

$$
\eta\left(\phi_{1}\right)=2 g^{2} \operatorname{Re}\left\langle\Omega_{0}^{*}-\Omega_{0}, \pi\left(v_{1}\right) i L_{r 1}\left(L_{0}+i 0\right)^{-1} e^{-\beta L_{0} / 2} \pi\left(v_{1}\right) \Omega_{0}\right\rangle+o\left(g^{2}\right) O(\delta \beta) .
$$

(12.3) follows by noticing that

$$
\operatorname{Re}\left\langle\Omega_{0}, \pi\left(v_{1}\right) i L_{r 1}\left(L_{0}+i 0\right)^{-1} e^{-\beta L_{0} / 2} \pi\left(v_{1}\right) \Omega_{0}\right\rangle=0 .
$$

Proof of Theorem 12.2. We want to control the components $\gamma_{j}$, appearing in the expression (12.5) for $\eta^{\prime}$. To this end we employ basic analytic perturbation theory (in $\delta \beta$ ) for the matrix family $M(\delta \beta):=\left.\Lambda_{0}^{*}(\delta \beta)\right|_{\beta_{p}=0}$, where we consider $\beta_{1}$ to be fixed. Write $\Gamma_{j}$ instead of $\Gamma_{j 0}$, see (10.3). According to Proposition 10.1 we have $M(\delta \beta)=M_{0}+\delta \beta M_{1}+O\left(\delta \beta^{2}\right)$, where

$$
\begin{aligned}
& M_{0}=-i\left(e^{-\beta_{1} H_{p} / 2} \otimes \mathbb{1}\right)\left\{\Gamma_{1}\left(\beta_{1}\right)+\Gamma_{2}\left(\beta_{1}\right)\right\}\left(e^{\beta_{1} H_{p} / 2} \otimes \mathbb{1}\right), \\
& M_{1}=-i\left(e^{-\beta_{1} H_{p} / 2} \otimes \mathbb{1}\right)\left\{\frac{1}{2}\left[H_{p} \otimes \mathbb{1}, \Gamma_{2}\left(\beta_{1}\right)\right]-\left(\partial_{\beta} \Gamma_{2}\right)\left(\beta_{1}\right)\right\}\left(e^{\beta_{1} H_{p} / 2} \otimes \mathbb{1}\right) .
\end{aligned}
$$


Let $\zeta^{*}=\sum_{j>0}(\delta \beta)^{j} \zeta_{j}^{*}$. The normalization $\left\langle\zeta^{*}, \zeta\right\rangle=1$ (where $\zeta=\Omega_{p}\left(\beta_{p}\right)$ is the particle Gibbs state) implies that $\left\langle\zeta_{0}^{*}, \zeta\right\rangle=1$ and $\left\langle\zeta_{j}^{*}, \zeta\right\rangle=0$, for $j \geq 1$. Solving the zero-oder eigenvalue equation $M_{0} \zeta_{0}^{*}=0$ gives

$$
\zeta_{0}^{*}=\sum_{j} \gamma_{j}^{(0)} \varphi_{j} \otimes \varphi_{j}, \quad \gamma_{j}^{(0)}=\frac{Z_{p}\left(\beta_{p}\right)}{Z_{p}\left(\beta_{1}+\beta_{p} / 2\right)} e^{-\beta_{1} E_{j}} .
$$

The first-order eigenvalue equation reads $M_{1} \zeta_{0}^{*}+M_{0} \zeta_{1}^{*}=0$, which implies

$$
\left[\Gamma_{1}\left(\beta_{1}\right)+\Gamma_{2}\left(\beta_{1}\right)\right]\left(e^{\beta_{1} H_{p} / 2} \otimes \mathbb{1}\right) \zeta_{1}^{*}=\left.\partial_{\beta}\right|_{\beta_{1}} \Gamma_{2}(\beta)\left(e^{\beta H_{p} / 2} \otimes \mathbb{1}\right) \zeta_{0}^{*} .
$$

We use here that $\left(e^{\beta_{1} H_{p} / 2} \otimes \mathbb{1}\right) \zeta_{0}^{*}$ is in the kernel of $H_{p}$. Let $\Psi(\beta):=\sum_{j} e^{-\beta E_{j} / 2} \varphi_{j} \otimes$ $\varphi_{j}$. Since $\Gamma_{2}(\beta) \Psi(\beta)=0$ we have $\left(\partial_{\beta} \Gamma_{2}\right)(\beta) \Psi(\beta)=-\Gamma_{2}(\beta)\left(\partial_{\beta} \Psi\right)(\beta)$, so

$$
\begin{aligned}
\left.\partial_{\beta}\right|_{\beta_{1}} \Gamma_{2}(\beta)\left(e^{\beta H_{p} / 2} \otimes \mathbb{1}\right) \zeta_{0}^{*} & =\left.C\left(\beta_{1}\right) \partial_{\beta}\right|_{\beta_{1} \Gamma_{2}(\beta) \Psi\left(2 \beta_{1}-\beta\right)} \\
& =C\left(\beta_{1}\right) \Gamma_{2}\left(\beta_{1}\right)\left(H_{p} \otimes \mathbb{1}\right) \Psi\left(\beta_{1}\right),
\end{aligned}
$$

where $C\left(\beta_{1}\right)=Z_{p}\left(\beta_{p}\right) / Z_{p}\left(\beta_{1}+\beta_{p} / 2\right)$. The r.h.s. of (12.30) is a vector in the orthogonal complement of $\operatorname{ker} \Gamma_{2}\left(\beta_{1}\right)=\mathbb{C} \Psi\left(\beta_{1}\right)$. Using this fact and (12.30) we solve (12.29) for $\zeta_{1}^{*}$ :

$$
\begin{aligned}
\zeta_{1}^{*}= & C\left(\beta_{1}\right)\left(e^{-\beta_{1} H_{p} / 2} \otimes \mathbb{1}\right)\left[\Gamma_{1}\left(\beta_{1}\right)+\Gamma_{2}\left(\beta_{1}\right)\right]^{-1} \Gamma_{2}\left(\beta_{1}\right)\left(H_{p} \otimes \mathbb{1}\right) \Psi\left(\beta_{1}\right) \\
& +C^{\prime}\left(e^{-\beta_{1} H_{p} / 2} \otimes \mathbb{1}\right) \Psi\left(\beta_{1}\right),
\end{aligned}
$$

where the constant $C^{\prime}$ is determined by the normalization condition $\left\langle\zeta_{1}^{*}, \zeta\right\rangle=0$. From expression (12.5) it is clear that the term (12.32) does not contribute to the value of $\eta^{\prime}$ (this is the same as saying that $\eta^{\prime}=0$ for $\delta \beta=0$ ).

Under the assumption $G_{1}=G_{2}=G$ we have $\Gamma_{1}\left(\beta_{1}\right)=\Gamma_{2}\left(\beta_{1}\right)$ and the r.h.s. of (12.31) simplifies to an easy expression, which, when used in (12.5), yields (12.6).

Proof of Theorem 3.2. If $G_{1}=G_{2}$ or if the dimension of the particle system is 2 then we have $\eta^{\prime}=\bar{\eta} \delta \beta+O\left((\delta \beta)^{2}\right)$ with $\bar{\eta}>0$ independent of $\delta \beta$. This follows from Theorem 12.2 in the case $G_{1}=G_{2}$ and from (11.22), (11.23) for the twodimensional case. In the latter case we use that, due to (12.5),

$$
\eta^{\prime}=\frac{2 \pi}{\alpha\left(E_{21}\right)+1}\left(e^{\beta_{1} E_{21}}-\alpha\left(E_{21}\right)\right) \frac{E_{21} g_{21}\left(E_{21}\right)^{2}}{e^{\beta_{1} E_{21}}-1}
$$

and we expand $e^{\beta_{1} E_{21}}-\alpha\left(E_{21}\right)$ around $\delta \beta=\beta_{1}-\beta_{2}=0$. Hence for $g$ and $\delta \beta$ both small, but independently of each other, we have, by (12.3), $E P\left(\eta_{\beta_{1} \beta_{2}}\right)>0$, which is the statement of Theorem 3.2.

Remark. A stronger statement, mentioned in the second paragraph after Theorem 3.2, can be proved as follows. By an abstract result of [19], $E P(\eta) \geq 0$. Therefore, due to (12.1), $\eta\left(\phi_{1}\right) \geq 0$ for $\beta_{1} \geq \beta_{2}$. Hence, due to (12.3), for $g$ sufficiently small (depending on $\delta \beta$ in general),

$$
\eta^{\prime} \geq 0 \text { for } \beta_{1} \geq \beta_{2} \text {. }
$$


Next, the $\gamma_{j}$ are analytic in $\beta_{1}$ and $\beta_{2}$ separately, away from $\beta_{1}=0, \beta_{2}=0$. To show this, we proceed as follows. From the explicit form of the level shift operator $\Lambda_{0}^{*}$, given in Proposition 10.1 and equation (10.5) and similar expressions for diagonal elements, we know that $\Lambda_{0}^{*}$ is analytic separately in $\beta_{1}$ and $\beta_{2}$, everywhere except for $\beta_{1}=0, \beta_{2}=0$. We also know that for each $\beta_{1}, \beta_{2}$ nonzero, $\Lambda_{0}^{*}$ has a simple eigenvalue at zero (since $\Lambda_{0}$ does). It follows from the Kato-Rellich theorem that we can find a zero-eigenvalue eigenvector $\zeta_{1}^{*}$, which is analytic in $\beta_{1}, \beta_{2}$. Next, we have to normalize that vector s.t. its overlap with $\Omega_{p}$ is unity. This yields $\zeta^{*}=\left\langle\zeta_{1}^{*}, \Omega_{p}\right\rangle^{-1} \zeta_{1}^{*}$. Since $\left\langle\zeta_{1}^{*}, \Omega_{p}\right\rangle$ cannot vanish, we have that $\zeta^{*}$ is analytic. Finally, the $\gamma_{j}$ of Theorem 12.1 are the components of $\zeta^{*}$, with $\beta_{p}=0$. From (11.18) we see that analyticity of the components of $\zeta^{*}$ in $\beta_{1}, \beta_{2}$ is true regardless of what $\beta_{p}$ is. Hence, the $\gamma_{j}$ are analytic separately in $\beta_{1}, \beta_{2}$, for $\beta_{1} \neq 0$, $\beta_{2} \neq 0$.

Analyticity of the $\gamma_{j}$ and expression (12.5) show that

$$
\eta^{\prime} \text { is analytic separately in } \beta_{1} \text { and } \beta_{2} \text {, for } \beta_{1} \neq 0, \beta_{2} \neq 0 \text {. }
$$

Equations (11.21) and (12.5) imply that

$$
\eta^{\prime}>0 \text { if } \beta_{1} \text { is fixed and } \beta_{2} \text { is sufficiently small. }
$$

Relations (12.33)-(12.35) imply $\eta\left(\phi_{1}\right)>0$ if $\beta_{1}>\beta_{2}$ for almost all values of $\left(\beta_{1}, \beta_{2}\right) \in(0, \infty) \times(0, \infty)$, in the sense that for fixed $\beta_{1} \in(0, \infty), \eta^{\prime}$ can vanish only for finitely many values of $\beta_{2}$ in any bounded subset of $(0, \infty)$. The same holds for $\beta_{1}$ and $\beta_{2}$ interchanged.

\section{Appendix A. Proof of existence of dynamics}

In this appendix we prove the existence of dynamics (2.12). Recall the definition of the operator $L^{(\ell)}:=L_{0}+g \pi(v)$ and the one-parameter group $\sigma^{t}(B):=$ $e^{i t L^{(\ell)}} B e^{-i t L^{(\ell)}}, B \in \pi(\mathcal{A})^{\prime \prime}$.

Proposition A.1. Assume the operators $v_{n} \in \mathcal{A}$ satisfy (2.13). Then for any state $\psi$ normal w.r.to $\omega_{0}$ the integrands on the r.h.s. of (2.12) are continuous functions, the series is absolutely convergent, the limit exists and equals

$$
\psi^{t}(A)=\operatorname{Tr}\left(\rho \sigma^{t}(\pi(A))\right)
$$

where $\rho$ is a positive, trace-class operator defined by $\psi(A):=\operatorname{Tr}(\rho \pi(A))$. In particular, $\psi^{t}(A)$ is independent of the approximating operators.

Proof. Let $v_{n} \in \mathcal{A}$ be an approximating sequence for the operator $v$ satisfying (2.13). We define the selfadjoint operators $L_{n}^{(\ell)}:=L_{0}+g \pi\left(v_{n}\right)$ on the dense domain $\mathcal{D}\left(L_{0}\right)$. Let the one parameter group $\sigma_{(n)}^{t}$ on $\pi(\mathcal{A})$ be given by

$$
\sigma_{(n)}^{t}(B):=e^{i t L_{n}^{(\ell)}} B e^{-i t L_{n}^{(\ell)}} .
$$


Set $\sigma_{0}^{t}(\pi(A)):=\pi\left(\alpha_{0}^{t}(A)\right)$ and let $\psi$ be an $\omega_{0}$-normal state on $\mathcal{A}$, i.e.,

$$
\psi(A)=\operatorname{Tr}(\rho \pi(A))
$$

for some positive, trace-class operator $\rho$ on $\mathcal{H}$ of trace 1 . Then using the definition $V_{n}=\pi\left(v_{n}\right)$ we find

$$
\psi\left(\left[\alpha_{0}^{t_{m}}\left(v_{n}\right), \cdots\left[\alpha_{0}^{t_{1}}\left(v_{n}\right), \alpha_{0}^{t}(A)\right] \cdots\right]\right)=\operatorname{Tr}\left(\rho\left[\sigma_{0}^{t_{m}}\left(V_{n}\right), \cdots\left[\sigma_{0}^{t_{1}}\left(V_{n}\right), \sigma_{0}^{t}(A)\right] \cdots\right]\right) .
$$

Clearly the r.h.s. is continuous in $t_{1}, \ldots, t_{m}$ and therefore the integrals in $(2.12)$ are well defined. Since the r.h.s. of (A.3) is bounded by $\left(c\left\|V_{n}\right\|\right)^{m}\left\|\sigma_{0}^{t}(A)\right\|$, the series on the r.h.s. of (2.12) converges absolutely. In fact, using the Araki-Dyson series

$$
\begin{aligned}
\sigma_{(n)}^{t}(\pi(A))= & \sum_{m=0}^{\infty}(i g)^{m} \int_{0}^{t} d t_{1} \cdots \int_{0}^{t_{m-1}} d t_{m}\left[\sigma_{0}^{t_{m}}\left(\pi\left(v_{n}\right)\right), \cdots\right. \\
& \left.\cdots\left[\sigma_{0}^{t_{1}}\left(\pi\left(v_{n}\right)\right), \sigma_{0}^{t}(\pi(A))\right] \cdots\right]
\end{aligned}
$$

one can easily see that the series in (2.12) is nothing but the Araki-Dyson expansion of the function $\operatorname{Tr}\left(\rho \sigma_{(n)}^{t}(\pi(A))\right)$. Thus we have shown that the r.h.s. of $(2.12)$ is equal to $\lim _{n \rightarrow \infty} \operatorname{Tr}\left(\rho \sigma_{(n)}^{t}(\pi(A))\right)$.

Now, $V_{n}$ converges to $V$ strongly on the dense set $\mathcal{C}:=\mathcal{H}_{p} \otimes \mathcal{H}_{p} \otimes$ $\operatorname{Span}\left\{\pi(A) \Omega_{0}\right\}$, where $A$ ranges over all polynomials in creation and annihilation operators $a_{j}^{*}(f), j=1,2$, with $f \in L_{0}^{2}$. This follows from (2.13) and the relation

$$
\left\|\left(V_{n}-V\right) \pi(A) \Omega_{0}\right\|^{2}=\omega_{0}\left(A^{*}\left(v_{n}^{*}-v^{*}\right)\left(v_{n}-v\right) A\right) .
$$

Hence $L_{n}^{(\ell)}$ converges to $L^{(\ell)}$ strongly on $\mathcal{C}$. The $\operatorname{set} \mathcal{C}$ is a core for both $L_{n}^{(\ell)}$ and $L^{(\ell)}$. (This can be seen by using the GJN commutator theorem, [13], Theorem 3.1, by taking $Y=\Lambda+N+\mathbb{1}$ for the comparison operator in this theorem, and by noticing that $\mathcal{C}$ is a core for $Y$. The latter fact follows from [30], Corollary 2 to Nelson's analytic vector theorem X.39.) It follows from Theorem VIII.25 of [29] that $L_{n}^{(\ell)}$ converges to $L^{(\ell)}$ in the strong resolvent sense as $n \rightarrow \infty$. In particular, $e^{i t L_{n}^{(\ell)}} \rightarrow e^{i t L^{(\ell)}}$ strongly, so $\operatorname{Tr}\left(\rho \sigma_{(n)}^{t}(\pi(A))\right) \rightarrow \operatorname{Tr}\left(\rho \sigma^{t}(\pi(A))\right)$ which, in particular, shows (A.1).

\section{Appendix B. Positive temperature representation}

\section{B.1. Gluing}

In this appendix, we represent the Hilbert space $\mathcal{H}$ in a form which is well suited for a definition of the translation transformation. This representation is due to [18].

Consider the Fock space

$$
\mathcal{F}:=\mathcal{F}\left(L^{2}(X \times\{1,2\})\right), \quad X=\mathbb{R} \times S^{2}
$$


and denote $x=(u, \sigma) \in X$. The vacuum in $\mathcal{F}$ is denoted by $\tilde{\Omega}_{r}$. The smeared-out creation operator $a^{*}(F), F \in L^{2}(X \times\{1,2\})$ is given by

$$
a^{*}(F)=\sum_{\alpha} \int_{X} F(x, \alpha) a^{*}(x, \alpha)
$$

and analogously for annihilation operators. The CCR read

$$
\left[a(x, \alpha), a^{*}\left(x^{\prime}, \alpha^{\prime}\right)\right]=\delta_{\alpha, \alpha^{\prime}} \delta\left(x-x^{\prime}\right) .
$$

Following [18], we introduce the unitary map

$U:\left[\mathcal{F}\left(L^{2}\left(\mathbb{R}^{3}\right)\right) \otimes \mathcal{F}\left(L^{2}\left(\mathbb{R}^{3}\right)\right)\right] \otimes\left[\mathcal{F}\left(L^{2}\left(\mathbb{R}^{3}\right)\right) \otimes \mathcal{F}\left(L^{2}\left(\mathbb{R}^{3}\right)\right)\right] \rightarrow \mathcal{F}\left(L^{2}(X \times\{1,2\})\right)$

defined by

$$
U\left(\left[\Omega_{r 1} \otimes \Omega_{r 1}\right] \otimes\left[\Omega_{r 2} \otimes \Omega_{r 2}\right]\right):=\tilde{\Omega}_{r}
$$

and

$$
\begin{aligned}
U\left(\left[a^{*}\left(f_{1}\right) \otimes \mathbf{1}\right.\right. & \left.+\mathbf{1} \otimes a^{*}\left(g_{1}\right)\right] \otimes \mathbf{1} \otimes \mathbf{1} \\
& \left.+\mathbf{1} \otimes \mathbf{1} \otimes\left[a^{*}\left(f_{2}\right) \otimes \mathbf{1}+\mathbf{1} \otimes a^{*}\left(g_{2}\right)\right]\right) U^{-1}:=a^{*}(f \oplus g),
\end{aligned}
$$

where, for $x=(u, \sigma) \in X$,

$$
[f \oplus g](u, \sigma, \alpha):= \begin{cases}u f_{\alpha}(u \sigma), & u \geq 0, \\ u g_{\alpha}(-u \sigma), & u<0 .\end{cases}
$$

This map is extended to the Hilbert space $\mathcal{H}=\mathcal{H}^{p} \otimes \mathcal{F}$ in the obvious way. We keep the same notation for its extension.

The operators $L_{r 1} \otimes \mathbf{1}_{r 2}+\mathbf{1}_{r 1} \otimes L_{r 2}$ and $N_{r 1} \otimes \mathbf{1}_{r 2}+\mathbf{1}_{r 1} \otimes N_{r 2}$ are mapped under $U$ to the (total) free field Liouvillian and number operator given by

$$
\begin{aligned}
L_{f} & =\mathrm{d} \Gamma(u)=\sum_{\alpha} \int_{X} a^{*}(x, \alpha) u a(x, \alpha), \\
N & =\mathrm{d} \Gamma(\mathbb{1})=\sum_{\alpha} \int_{X} a^{*}(x, \alpha) a(x, \alpha) .
\end{aligned}
$$

Moreover, the interaction takes the form

$$
U I U^{-1}=a^{*}\left(F_{1}\right)+a\left(F_{2}\right),
$$


where $F_{1,2} \in L^{2}\left(X \times\{1,2\}, \mathcal{B}\left(\mathcal{H}_{p} \otimes \mathcal{H}_{p}\right)\right)$ are explicitly given by $(x=(u, \sigma) \in X=$ $\left.\mathbb{R} \times S^{2}\right)$

$$
\begin{aligned}
& F_{1}(u, \sigma, \alpha)=\sqrt{\frac{u}{1-e^{-\beta_{\alpha} u}}} \\
& \quad \times\left\{\begin{array}{lr}
\sqrt{u}\left(G_{\alpha}(u \sigma) \otimes \mathbb{1}_{p}-e^{-\beta_{\alpha} u / 2} e^{\delta \beta_{\alpha} u / 2} \mathbb{1}_{p} \otimes \alpha_{p}^{i \delta \beta_{p} / 2}\left({\overline{G_{\alpha}}}^{*}(u \sigma)\right)\right), & u>0 \\
-\sqrt{-u}\left(G_{\alpha}^{*}(-u \sigma) \otimes \mathbb{1}_{p}-e^{-\beta_{\alpha} u / 2} e^{\delta \beta_{\alpha} u / 2} \mathbb{1}_{p} \otimes \alpha_{p}^{i \delta \beta_{p} / 2}\left(\overline{G_{\alpha}}(-u \sigma)\right)\right), & u<0
\end{array}\right.
\end{aligned}
$$

and

$$
\begin{aligned}
& F_{2}(u, \sigma, \alpha)=\sqrt{\frac{u}{1-e^{-\beta_{\alpha} u}}} \\
& \quad \times\left\{\begin{array}{lr}
\sqrt{u}\left(G_{\alpha}(u \sigma) \otimes \mathbb{1}_{p}-e^{-\beta_{\alpha} u / 2} e^{-\delta \beta_{\alpha} u / 2} \mathbb{1}_{p} \otimes \alpha_{p}^{-i \delta \beta_{p} / 2}\left({\overline{G_{\alpha}}}^{*}(u \sigma)\right)\right), & \text { (B.1.8) } \\
-\sqrt{-u}\left(G_{\alpha}^{*}(-u \sigma) \otimes \mathbb{1}_{p}-e^{-\beta_{\alpha} u / 2} e^{-\delta \beta_{\alpha} u / 2} \mathbb{1}_{p} \otimes \alpha_{p}^{-i \delta \beta_{p} / 2}\left(\overline{G_{\alpha}}(-u \sigma)\right)\right), & u<0
\end{array}\right.
\end{aligned}
$$

\section{B.2. Complex deformation}

Now we express the complex deformation operators $U_{\theta}$ introduced in Section 6 in the glued Hilbert space. For a function $F \in L^{2}(X \times\{1,2\})$ and $\theta=(\delta, \tau)$, $x=(u, \sigma) \in X$, define

$$
\left[\tilde{u}_{\theta} F\right](u, \sigma, \alpha)=e^{\frac{1}{2} \delta \operatorname{sgn}(u)} F\left(j_{\theta}(u), \sigma, \alpha\right),
$$

where

$$
j_{\theta}(u)=e^{\delta \operatorname{sgn}(u)} u+\tau,
$$

and $\operatorname{sgn}$ is the sign function, $\operatorname{sgn}(u)=1$ if $u \geq 0, \operatorname{sgn}(-u)=-\operatorname{sgn}(u)$. Next, we lift the operator family $\tilde{u}_{\theta}$ from $L^{2}(X \times\{1,2\})$ to the operator family, $\tilde{U}_{\theta}$, on $\mathcal{H}^{p} \otimes \mathcal{F}\left(L^{2}(X \times\{1,2\})\right)$ in a standard way (cf. (6.1)). The family $\tilde{U}_{\theta}$ is related to the family $U_{\theta}$ introduced in Section 6 as

$$
U_{\theta}=U^{-1} \tilde{U}_{\theta} U .
$$

The operator $\tilde{K}:=U K U^{-1}$ becomes after spectral deformation

$$
\tilde{K}_{\theta}:=\tilde{U}_{\theta} K \tilde{U}_{\theta}^{-1}=\tilde{L}_{0, \theta}+g \tilde{I}_{\theta}
$$

where

$$
\begin{aligned}
\tilde{L}_{0, \theta} & =L_{p}+\cosh \delta L_{f}+\sinh \delta \Lambda_{f}+\tau N, \\
\Lambda_{f} & =\mathrm{d} \Gamma(|u|)=\sum_{\alpha} \int_{X} a^{*}(x, \alpha)|u| a(x, \alpha), \\
\tilde{I}_{\theta} & =a^{*}\left(F_{1, \theta}\right)+a\left(F_{2, \theta}\right) \quad \text { with } \quad F_{j, \theta}=\tilde{u}_{\theta} F_{j} .
\end{aligned}
$$


This spectral deformation can be translated to the original space $\mathcal{H}$ as

$$
K_{\theta}:=U \tilde{K}_{\theta} U^{-1}=L_{0, \theta}+g I_{\theta}
$$

where $L_{0, \theta}:=U^{-1} \tilde{L}_{0, \theta} U$ is given by (6.14) and

$$
I_{\theta}=U^{-1} \tilde{I}_{\theta} U \text {. }
$$

\section{Appendix C. The $C^{*}$-algebra $\mathcal{A}_{1}$}

Proposition C.1. Let the algebras $\mathcal{A}_{1}$ and $\mathcal{A}$ be defined by $(2.17)$ with $\mathcal{D}^{\text {anal }}$ given in the proof below, and (2.7), respectively. Then $\mathcal{A}_{1}$ is strongly dense in $\mathcal{A}$.

Proof. Let $L_{1}^{2}=\left\{f\right.$ a.e. continuous, $\left.\|f\|_{L_{1}^{2}}^{2}:=\int|f(k)|^{2}\left(|k|^{-1}+1\right) d^{3} k<\infty\right\}$. Recall the real linear map $\widetilde{\gamma}_{\beta}$ defined in (3.7). Using that for $x \geq 0, \max (1 / x, 1) \leq$ $\frac{1+e^{-x}}{1-e^{-x}} \leq 4 \max (1 / x, 1)$, we obtain

$$
c\|f\|_{L_{1}^{2}}^{2} \leq\left\|\widetilde{\gamma}_{\beta} f\right\|_{L^{2}}^{2}=\int_{S^{2}} d \sigma \int_{0}^{\infty} d u \frac{1+e^{-\beta u}}{1-e^{-\beta u}} u^{2}|f(u \sigma)|^{2} \leq C\|f\|_{L_{1}^{2}}^{2},
$$

so $\widetilde{\gamma}_{\beta}$ is bounded and invertible (on $R:=\widetilde{\gamma}_{\beta} L_{1}^{2} \subset L^{2}$ ), and $\widetilde{\gamma}_{\beta}^{-1}$ is a (real linear) bounded map. We have the equivalence

$$
\begin{aligned}
g \in R \Leftrightarrow & g(u, \sigma)=-e^{\beta u / 2} \bar{g}(-u, \sigma) \quad \text { for a.e. } u \in \mathbb{R} \\
& \text { and } \int_{\mathbb{R}} d u \int_{S^{2}} d \sigma|g(u, \sigma)|^{2}<\infty .
\end{aligned}
$$

Let $R_{0}:=\left\{g \in R, e^{b u^{2}} g \in L^{2}\right.$ for some $\left.b \geq 0\right\} \subset R$. The set $R_{0}$ is dense in $R$ and (C.2) implies that

$$
R_{0}=\left\{e^{\beta u / 4} h \mid: e^{b u^{2}} h \in L^{2} \text { for some } b>0 \text { and } h(u, \sigma)=-\bar{h}(-u, \sigma)\right\} .
$$

Given $g=e^{\beta u / 4} h \in R_{0}$, define $h_{\epsilon}:=G_{\epsilon} * h$, the convolution in the variable $u$ of $h$ with the Gaussian $G_{\epsilon}(u):=\epsilon^{-1} G(u / \epsilon)$, where $G(u)=\pi^{-1 / 2} e^{-u^{2}}$ and $\epsilon>0 . h_{\epsilon}$ is continuous (actually analytic), satisfies $e^{b u^{2}} h_{\epsilon} \in L^{2}$, and since $G_{\epsilon}(\cdot)$ is real valued and odd:

$$
h_{\epsilon}(u, \sigma)=-\bar{h}_{\epsilon}(-u, \sigma) .
$$

Therefore, $g_{\epsilon}:=e^{\beta u / 4} h_{\epsilon} \in R_{0}$. Since $h_{\epsilon} \rightarrow h$ in $L^{2}$, we conclude that $g_{\epsilon} \rightarrow g$ in $L^{2}$ as $\epsilon \rightarrow 0$. Clearly, $g_{\epsilon}$ extends to an entire function $z \mapsto g_{\epsilon}(z, \sigma)$. Define the set

$$
R^{\text {anal }}:=\left\{e^{\beta u / 4} h_{\epsilon} \mid h \text { satisfies the conditions on r.h.s. of (C.3), } \epsilon>0\right\} .
$$

$R^{\text {anal }}$ is a subset of $R$ that is dense in $R$. Since $\widetilde{\gamma}_{\beta}^{-1}$ is bounded, then $D^{\text {anal }}:=$ $\widetilde{\gamma}_{\beta}^{-1}\left(R^{\text {anal }}\right)$ is dense in $L_{1}^{2}$. Since $L_{1}^{2}$ is dense in $L^{2}$, we conclude that $D^{\text {anal }}$ is also dense in $L^{2}$.

Define $\mathcal{A}_{1}$ as in $(2.17)$ with $\mathcal{D}^{\text {anal }}$ given above. Since $\mathcal{D}^{\text {anal }}$ is dense in $L^{2}, \mathcal{A}_{1}$ is strongly dense in $\mathcal{A}$ (defined by (2.7)). Next we have for real $\theta$

$$
U_{\theta} \pi\left(A_{p} \otimes W\left(f_{1}\right) \otimes W\left(f_{2}\right)\right) \Omega=\pi_{p}\left(A_{p}\right) \otimes W\left(\left(\widetilde{\gamma}_{\beta} f_{1}\right)_{\theta}\right) \otimes W\left(\left(\widetilde{\gamma}_{\beta} f_{2}\right)_{\theta}\right) \Omega_{\theta}
$$


where the map $g \rightarrow g_{\theta}$ is defined by $g_{\theta}(u, \sigma):=g\left(j_{\theta}(u), \sigma\right)$ with the function $j_{\theta}$ defined in (A2.2) and where we understand the Weyl operators on the r.h.s. as acting on the (glued) GNS space and $\Omega_{\theta}$ given in the same representation. Using that $\theta \rightarrow\left(\widetilde{\gamma}_{\beta} f_{j}\right)_{\theta}$ are analytic for $f_{j} \in \mathcal{D}^{\text {anal }}$ and $\left(\widetilde{\gamma}_{\beta} f_{j}\right)_{\theta} \in L^{2}\left(\mathbb{R} \times S^{2}\right)$ as long as $|\tan (\operatorname{Im} \delta)|<\frac{b}{1+b}$ and expanding $W\left(\left(\widetilde{\gamma}_{\beta} f_{j}\right)_{\theta}\right)$ into the Taylor series and $\Omega_{\theta}$ into the Dayson-Araki one, one can show the r.h.s. of (C.4) has an analytic continuation in $\theta$ into the neighbourhood of $\mathbb{R}$ given by

$$
\left\{\theta \in \mathbb{C}^{2}|| \tan (\operatorname{Im} \delta) \mid<\frac{b}{1+b} \quad \text { and } \quad|\operatorname{Im} \tau|<\beta^{-1}\right\} .
$$

\section{Appendix D. The vectors $\zeta^{*}$}

In this appendix, we outline the calculation of the vectors $\zeta^{*}$ in the special cases mentioned in (11.18)-(11.23). As mentioned after (11.18), we may restrict our attention to $\beta_{p}=0$.

(i) $\beta_{1}=\beta_{2}=\beta$. Proposition 10.1 gives

$$
\Lambda_{0}^{*}=\left[e^{-\beta H_{p} / 2} \otimes \mathbb{1}\right]\left(\Lambda_{10}^{*}+\Lambda_{20}^{*}\right)\left[e^{\beta H_{p} / 2} \otimes \mathbb{1}\right] .
$$

Therefore, $\left[e^{\beta H_{p} / 2} \otimes \mathbb{1}\right] \zeta^{*}$ must be in the kernel of $\Lambda_{10}^{*}+\Lambda_{20}^{*}$, which is spanned by $\Omega_{p}^{\beta}$. Thus

$$
\zeta^{*} \propto\left[e^{-\beta H_{p} / 2} \otimes \mathbb{1}\right] \sum_{j} e^{-\beta E_{j} / 2} \varphi_{j} \otimes \varphi_{j}=\sum_{j} e^{-\beta E_{j}} \varphi_{j} \otimes \varphi_{j} .
$$

The normalization is given by setting $\left\langle\Omega_{p}^{\left(\beta_{p}=0\right)}, \zeta^{*}\right\rangle=1$. This yields the expression (11.20).

(ii) Proposition 10.1 and equation (10.3) imply that

$$
\Lambda_{0}^{*}=i \Gamma_{10}+O(1)
$$

where the operator $O(1)$ is bounded as $\beta_{2} \rightarrow 0$. The matrix elements of $\Gamma_{10}=\Gamma_{10}\left(\beta_{2}\right)$ in the basis $\left\{\varphi_{n} \otimes \varphi_{n}\right\}$ are (see [8], equations (B21)-(B22))

$$
\left(\Gamma_{10}\right)_{m, n}=\delta_{m, n} \sum_{k=0, k \neq m} e^{\beta_{2} E_{m k} / 2} \eta_{m k}-\left(1-\delta_{m n}\right) \eta_{m n},
$$

where $\delta_{m n}$ is the Kronecker symbol, and where

$$
\eta_{m n}=2 \pi E_{m n}^{2} \frac{e^{\beta_{2}\left|E_{m n}\right| / 2}}{e^{\beta_{2}\left|E_{m n}\right|}-1} \int_{S^{2}} d \sigma\left|G_{2}\left(\left|E_{m n}\right|, \sigma\right)_{m n}\right|^{2} .
$$

We expand $\Gamma_{10}$ in $\beta_{2}$ :

$$
\Gamma_{10}=\frac{1}{\beta_{2}} \Gamma^{\prime}+O(1)
$$


where $O(1)$ is bounded as $\beta_{2} \rightarrow 0$, and where

$$
\begin{aligned}
\left(\Gamma^{\prime}\right)_{m, n}= & 2 \pi \delta_{m, n} \sum_{k=0, k \neq m}\left|E_{m k}\right| \int_{S^{2}} d \sigma\left|G_{2}\left(\left|E_{m k}\right|, \sigma\right)_{m, k}\right|^{2} \\
& -2 \pi\left(1-\delta_{m n}\right)\left|E_{m n}\right| \int_{S^{2}} d \sigma\left|G_{2}\left(\left|E_{m n}\right|, \sigma\right)_{m, n}\right|^{2} .
\end{aligned}
$$

It is obvious that the vector with constant coordinates $[1,1, \ldots, 1]^{T}$ is in the kernel of $\Gamma^{\prime}$, and, by the Perron-Frobenius theorem, that zero is a simple eigenvalue of $\Gamma^{\prime}$.

Hence, we see from (D.1) and (D.2), by perturbation theory, that the vector in the kernel of $\Lambda_{0}^{*}$ is of the form $\zeta^{*} \propto[1,1, \ldots, 1]^{T}+O\left(\beta_{2}\right)$, as $\beta_{2} \rightarrow 0$. The proper normalization $\left\langle\Omega_{p}^{\left(\beta_{p}=0\right)}, \zeta^{*}\right\rangle=1$ yields (11.21).

(iii) $\operatorname{dim} \mathcal{H}_{p}=2$. Let $E=E_{2}-E_{1}>0$. We use Proposition 10.1 and formula (10.5) to obtain the following expression for the level shift operator $\Lambda_{0}$ :

$$
\Lambda_{0}=2 \pi i E^{2}\left[\begin{array}{cc}
a & -a \\
-b & b
\end{array}\right]
$$

where

$$
\begin{aligned}
a & =\sum_{j=1,2}\left(e^{\beta_{j} E}-1\right)^{-1} \int_{S^{2}} d \sigma\left|\left[G_{j}(E, \sigma)\right]_{12}\right|^{2}, \\
b & =\sum_{j=1,2} \frac{e^{\beta_{j} E}}{e^{\beta_{j} E}-1} \int_{S^{2}} d \sigma\left|\left[G_{j}(E, \sigma)\right]_{12}\right|^{2} .
\end{aligned}
$$

It is easily seen that $\Omega_{p}^{\left(\beta_{p}=0\right)} \propto[1,1]^{T}$ is in the kernel of $\Lambda_{0}$, as it should be. The eigenvalues of $\Lambda_{0}$ are thus zero and $\operatorname{Tr} \Lambda_{0}=2 \pi i E^{2}(a+b) \neq 0$. The kernel of $\Lambda_{0}^{*}$ is spanned by $\mathbb{C}[b / a, 1]^{T}$, so $\zeta^{*} \propto[b / a, 1]^{T}=\frac{b}{a} \varphi_{1} \otimes \varphi_{1}+\varphi_{2} \otimes \varphi_{2}$. The normalization is given by setting $\left\langle\Omega_{p}^{\left(\beta_{p}=0\right)}, \zeta^{*}\right\rangle=1$. This yields the expressions (11.22), (11.23).

\section{Acknowledgements}

The authors are grateful to V. Bach, G. Elliott, J. Fröhlich, and especially C.-A. Pillet for useful discussions, and to V. Jakšić and C.-A. Pillet, for pointing out numerous inaccuracies and typos and the desirability of more details in the original manuscript. Part of this work was done while the first author was visiting the University of Toronto, the third author, ETH, Zürich and the second and third authors visited ESI Vienna. During work on this paper the second author was at the University of Toronto on a DAAD fellowship. The authors are grateful to these places for hospitality. 


\section{References}

[1] W. K. Abou Salem, On the quasi-static evolution of non-equilibrium steady states, Preprint, mp-arc 05-341.

[2] W. K. Abou Salem and J. Fröhlich, Cyclic thermodynamic processes and entropy production, Preprint, mp-arc 05-436.

[3] H. Araki and E. J. Woods, Representation of the canonical commutation relations describing a nonrelativistic infinite free bose gas, J. Math. Phys. 4 (1963), 637-662.

[4] W. Aschbacher and C.-A. Pillet, Non-equilibrium steady states of the XY chain, J. Statist. Phys. 112 (2003), no. 5-6, 1153-1175.

[5] V. Bach, T. Chen, J. Fröhlich, and I. M. Sigal, Smooth Feshbach map and operator theoric renormalization group method, J. Func. Analysis 203 (2003).

[6] V. Bach, J. Fröhlich, and I. M. Sigal, Quantum electrodynamics of confined nonrelativistic particles, Adv. in Math. 137 (1998), 299-395.

[7] V. Bach, J. Fröhlich, and I. M. Sigal, Renormalization group analysis of spectral problems in quantum field theory, Adv. in Math. 137 (1998), 205-298.

[8] V. Bach, J. Fröhlich, and I. M. Sigal, Return to equilibrium, J. Math. Phys. 41 (2000), 3985-4060.

[9] O. Bratteli and D.W. Robinson, Operator Algebras and Quantum Statistical Mechanics, volumes 1 and 2. Springer Verlag, New York, Heidelberg, Berlin, 1979.

[10] J. Dereziński and V. Jakšić, Spectral theory of Pauli-Fierz operators, J. Func. Anal. 180 (2001), 243.

[11] J. Dereziński, V. Jakšić, and C.-A. Pillet, Perturbation theory of $W^{*}$-dynamics, Liouvilleans and KMS-states, Rev. Math. Phys. 15 (2003), no. 5, 447-489.

[12] S. Dirren, Dobrushin's Uniqueness Theorem, High Temperature Expansions, ETH Zürich Diploma Thesis, written under supervision of J. Fröhlich and G. M. Graf, 1999.

[13] J. Fröhlich and M. Merkli, Thermal ionization, Mathematical Physics, Analysis and Geometry 7, no. 3 (2004), 239-287.

[14] J. Fröhlich and M. Merkli, Another Return of "Return to Equilibrium", Commun. Math. Phys. 251 (2004), 235-262.

[15] J. Fröhlich, M. Merkli, and D. Ueltschi, Dissipative transport: Thermal contacts and tunneling junctions, Annales Henri Poincaré 4, no. 5 (2004), 897-945.

[16] R. Haag, Local Quantum Physics, Texts and Monographs in Physics, Springer Verlag, 1992.

[17] R. Haag, N. Hugenholz, and M. Winnink, On the equilibrium states in quantum statistical mechanics, Comm. Math. Phys. 5 (1967), 215-236.

[18] V. Jakšić and C.-A. Pillet, On a model for quantum friction II. Fermi's golden rule and dynamics at positive temperature, Comm. Math. Phys. 176 (1996), 619-644.

[19] V. Jakšić and C.-A. Pillet, On entropy production in quantum statistical mechanics, Comm. Math. Phys. 217 (2001), 285-293.

[20] V. Jakšić and C.-A. Pillet, Mathematical theory of non-equilibrium quantum statistical mechanics, J. Stat. Phys. 108 (2002), 787-829.

[21] V. Jakšić and C.-A. Pillet, Non-equilibrium steady states of finite quantum systems coupled to thermal reservoirs, Comm. Math. Phys. 226 (2002), 131-162. 
[22] M. Merkli, Positive commutators in non-equilibrium statistical mechanics, Comm. Math. Phys. 223 (2001), 327-362.

[23] M. Merkli, Stability of Equilibria with a Condensate, Comm. Math. Phys. 257, no. 3 (2005), 621-640.

[24] M. Merkli, Level shift operators for open quantum systems, J. Math. Anal. Appl. 327, no. 1 (2007), 376-399.

[25] M. Merkli, M. Mück, I. M. Sigal, Instability of Equilibrium States for Coupled Heat Reservoirs at Different Temperatures, J. Funct. Anal. 243, no. 1 (2007), 87-120.

[26] M. Mück, Thermal Relaxation for Particle Systems in Interaction with Several Bosonic Heat Reservoirs, Ph.D. Dissertation, Department of Mathematics, Johannes-Gutenberg University, Mainz, July 2004, ISBN 3-8334-1866-4.

[27] I. Ojima, H. Hasegawa and M. Ichiyanagi, Entropy production and its positivity in nonlinear response theory of quantum dynamical systems, J. Stat. Phys. 50 (1998), 633-655.

[28] I. Ojima, Entropy production and nonequilibrium stationarity in quantum dynamical systems. Physical meaning of van Hove limit, J. Stat. Phys. 56 (1989), 203-226.

[29] M. Reed, B. Simon, Methods of Modern Mathematical Physics I, Functional Analysis, Academic Press, 1980.

[30] M. Reed, B. Simon, Methods of Modern Mathematical Physics II, Fourier Analysis, Self-adjointness, Academic Press, 1980.

[31] D. Ruelle, Statistical Mechanics. Rigorous Results. Benjamin, New York, 1969.

[32] D. Ruelle, Natural non-equilibrium states in quantum statistical mechanics, J. Stat. Phys. 98 (2000), 57.

[33] D. Ruelle, Entropy production in quantum spin systems, Comm. Math. Phys. 224 (2001), 3 .

[34] D. Ruelle, How should one define entropy production for non-equilibrium quantum spin systems? Preprint (2001).

[35] D. Ruelle, Topics in quantum statistical mechanics and operator algebras, Preprint (2001).

[36] H. Spohn, J. L. Lebowitz, Irreversible thermodynamics for quantum systems weakly coupled to thermal reservoirs, Advances in Chemical Physics 38 (1978), 109-142.

Marco Merkli

Department of Mathematics and Statistics

Memorial University of Newfoundland

St. John's, NL, A1C 5S7

Canada

e-mail: merkli@math.mun.ca

Matthias Mück

Fachbereich Mathematik

Johannes Gutenberg-Universität

D-55128 Mainz

Germany

e-mail: mueck@mathematik.uni-mainz.de 
Israel Michael Sigal

Department of Mathematics

University of Toronto

Toronto, ON, M5S 2E4

Canada

e-mail: im.sigal@utoronto.ca

Communicated by Vincent Rivasseau.

Submitted: March 20, 2006.

Revised: March 19, 2007.

Accepted: May 11, 2007. 